\title{
The connection between galaxy environment and the luminosity function slopes of star-forming regions
}

\author{
David O. Cook, ${ }^{1,2 \star}$ Daniel A. Dale, ${ }^{1 \star}$ Janice C. Lee,${ }^{3 \star}$ David Thilker, ${ }^{3}$ \\ Daniela Calzetti ${ }^{4}$ and Robert C. Kennicutt ${ }^{5}$ \\ ${ }^{1}$ Department of Physics and Astronomy, University of Wyoming, Laramie, WY 82071, USA \\ ${ }^{2}$ California Institute of Technology, 1200 East California Blvd, Pasadena, CA 91125, USA \\ ${ }^{3}$ Space Telescope Science Institute, 3700 San Martin Drive, Baltimore, MD 21218, USA \\ ${ }^{4}$ Department of Astronomy, University of Massachusetts, Amherst, MA 01003, USA \\ ${ }^{5}$ Institute of Astronomy, University of Cambridge, Cambridge CB3 OHA, UK
}

Accepted 2016 July 12. Received 2016 July 2; in original form 2016 March 10

\begin{abstract}
We present the first study of GALEX far-ultraviolet (FUV) luminosity functions of individual star-forming regions within a sample of 258 nearby galaxies spanning a large range in total stellar mass and star formation properties. We identify $\sim 65000$ star-forming regions (i.e. FUV sources), measure each galaxy's luminosity function, and characterize the relationships between the luminosity function slope $(\alpha)$ and several global galaxy properties. A final sample of 82 galaxies with reliable luminosity functions are used to define these relationships and represent the largest sample of galaxies with the largest range of galaxy properties used to study the connection between luminosity function properties and galaxy environment. We find that $\alpha$ correlates with global star formation properties, where galaxies with higher star formation rates and star formation rate densities $\left(\Sigma_{\mathrm{SFR}}\right)$ tend to have flatter luminosity function slopes. In addition, we find that neither stochastic sampling of the luminosity function in galaxies with low-number statistics nor the effects of blending due to distance can fully account for these trends. We hypothesize that the flatter slopes in high $\Sigma_{\text {SFR }}$ galaxies is due to higher gas densities and higher star formation efficiencies which result in proportionally greater numbers of bright star-forming regions. Finally, we create a composite luminosity function composed of star-forming regions from many galaxies and find a break in the luminosity function at brighter luminosities. However, we find that this break is an artefact of varying detection limits for galaxies at different distances.
\end{abstract}

Key words: galaxies: dwarf-galaxies: irregular-Local Group-galaxies: spiral-galaxies: star clusters: general-galaxies: star formation.

\section{INTRODUCTION}

A galaxy's ultraviolet (UV) flux traces young, massive stars and thus is a good indicator of recent star formation [i.e. far-ultraviolet (FUV) traces $t<100$ Myr; Kennicutt 1998; Murphy et al. 2011. Furthermore, clustering studies of young stars and star clusters have shown that star formation occurs in a clustered environment $(t<100$ Myr; Lada \& Lada 2003; Gouliermis et al. 2010, 2015), thus FUV sources with relatively low angular resolution (a few arcseconds) will trace groups of young, massive stars that formed at similar times and physical locations (i.e. star-forming regions).

^E-mail: dcook@astro.caltech.edu (DOC); ddale@uwyo.edu (DAD); jlee@stsci.edu (JCL)
The star formation process shows evidence of a fractal, or scalefree, structure where the distributions of stars, star clusters, and stellar complexes show similar patterns on different physical scales [see review and references in Elmegreen (2010)]. As a consequence of this fractal picture, the mass function (and similarly the luminosity function; LF) of these distributions can be represented as a power law characterized by a slope of -2 (Elmegreen 2006). Observationally, the mass and LFs of star-forming regions (i.e. star clusters and $\mathrm{H}$ II regions) have shown to be adequately approximated as a power law with a slope of $-2 \pm 0.2$ (Zhang \& Fall 1999; Larsen 2002; Bik et al. 2003; de Grijs et al. 2003; Hunter et al. 2003; McCrady \& Graham 2007; Cook et al. 2012; Whitmore et al. 2014; Chandar et al. 2015; Adamo et al., in preparation). However, it is not clear if galaxy environment has a significant effect on $\alpha$, nor is the scatter well understood. 
Many star cluster and $\mathrm{H}$ II region studies that measure mass function (MF)/LFs do so with small galaxy samples (usually only a few), and the methods used to identify regions and to generate MF/LFs can vary from study to study. The inhomogeneity of data and methods can add scatter to any relationship of MF/LF slope with galaxy properties and possibly mask any correlations. However, mass and LFs derived for multiple galaxies with uniform data and methods have found hints of systematic trends between $\alpha$ and global galaxy properties [e.g. star formation rate (SFR), $\mathrm{M}_{\mathrm{B}}$, galaxy type, etc.; Kennicutt et al. 1989; Elmegreen \& Salzer 1999; Youngblood \& Hunter 1999; van Zee 2000; Thilker et al. 2002; Whitmore et al. 2014. These trends suggest that environment may play a role in the formation of stars and the quantification of such trends would provide clues into the star formation process.

In addition to environmental effects on the star formation process, mass and LFs have shown evidence for a break in their fitted power laws at higher masses (e.g. $\mathrm{M}_{\text {star }} \sim 10^{5}-10^{6} \mathrm{M}_{\odot}$; Gieles et al. 2006; Bastian et al. 2012; Adamo et al., in preparation) and luminosities (e.g. $\mathrm{L}_{\mathrm{H} \alpha} \sim 38.6 \mathrm{erg} \mathrm{s}^{-1}$; Kennicutt et al. 1989; Pleuss et al. 2000; Bradley et al. 2006, see also Whitmore et al. 2014, Adamo et al., in preparation), where the slope is steeper at higher masses/luminosities. This break has been interpreted as a truncation in the mass function due to density bounded high-luminosity regions suggesting a possible upper limit on the mass of star-forming regions (Beckman et al. 2000).

Since the measurements of the masses and luminosities of bright star-forming regions required to accurately characterize this break are relatively rare even in normal star-forming galaxies (Larsen 2002; Gieles et al. 2006; Bastian 2008), studies have constructed composite LFs that include the regions of many galaxies to increase the number statistics of bright, star-forming regions (Bradley et al. 2006; Whitmore et al. 2014). However, the galaxy samples used span a range of distance $(\sim 10 \mathrm{~s}$ of $\mathrm{Mpc})$ which may contribute to an artificial break due to different luminosity detection limits of galaxies at different distances.

This paper is the first of two papers which aim to test the universal nature of the MF/LF of star-forming regions in a sample of 258 nearby galaxies whose properties span a wide range in SFR, metallicity, luminosity, and Hubble type. The current paper will look at the FUV LFs of star-forming regions while the second paper will look at the MFs. Specifically, this paper measures the LFs, quantifies and trends between LF slope and global galaxy properties, and investigates a break in the composite LF containing star-forming regions from many galaxies.

\section{DATA AND SAMPLE}

The local volume legacy (LVL) sample consists of 258 of our nearest galaxy neighbours reflecting a statistically complete, representative sample of the local universe. The sample selection and description are detailed in Dale et al. (2009), but we provide a brief overview here.

The LVL galaxy sample was built upon a panchromatic data set covering UV, optical, and infrared (IR) wavelengths with the aim of studying both obscured and unobscured star formation in the Local Universe. The final LVL sample consists of galaxies that appear outside the Galactic plane $\left(|b|>20^{\circ}\right)$, have a distance less than $11 \mathrm{Mpc}(D \leq 11 \mathrm{Mpc})$, span an absolute $B$-band magnitude of $-9.6<M_{\mathrm{B}}<-20.7$, and span an RC3 catalogue galaxy type range of $-5<T<10$. Although the galaxy morphology composition is diverse, the LVL sample is dominated by dwarf galaxies due to its
Table 1. General galaxy properties.

\begin{tabular}{lcccc}
\hline $\begin{array}{l}\text { Galaxy } \\
\text { Name } \\
(1)\end{array}$ & $\begin{array}{c}\text { RA } \\
(\mathrm{J} 2000.0)\end{array}$ & $\begin{array}{c}\text { Dec } \\
(\mathrm{J} 2000.0)\end{array}$ & $\begin{array}{c}D \\
(\mathrm{Mpc})\end{array}$ & $T$ \\
\hline WLM & $(2)$ & $(3)$ & $(4)$ & $(5)$ \\
NGC0024 & $00: 01: 58.16$ & $-15: 27: 39.3$ & 0.92 & 10 \\
NGC0045 & $00: 09: 56.54$ & $-24: 57: 47.3$ & 8.13 & 5 \\
NGC0055 & $00: 14: 03.99$ & $-23: 10: 55.5$ & 7.07 & 8 \\
NGC0059 & $00: 14: 53.60$ & $-39: 11: 47.9$ & 2.17 & 9 \\
ESO410-G005 & $00: 15: 25.13$ & $-21: 26: 39.8$ & 5.30 & -3 \\
SCULPTOR-DE1 & $00: 15: 31.56$ & $-32: 10: 47.8$ & 1.90 & -1 \\
ESO294-G010 & $00: 23: 51.70$ & $-24: 42: 18.0$ & 4.20 & 10 \\
IC1574 & $00: 26: 33.37$ & $-41: 51: 19.1$ & 1.90 & -3 \\
NGC0247 & $00: 43: 03.82$ & $-22: 14: 48.8$ & 4.92 & 10 \\
\hline
\end{tabular}

Column 1: Galaxy name. Columns 2 and 3: J2000 right ascension and declination from the R25 apertures of Cook et al. (2014a). Column 4: distance in Mpc from Kennicutt et al. (2008). Column 5: RC3 Morphological T-type from Kennicutt et al. (2008). The full table is available online.

volume-limited nature. The full LVL sample and basic properties are listed in Table 1.

The published data sets of LVL consist of GALEX UV (Lee et al. 2011), Spitzer IR and 2MASS NIR (Dale et al. 2009), ground-based optical (Cook et al. 2014a), and ground-based $\mathrm{H} \alpha$ (Kennicutt et al. 2008 ) imaging. We use the GALEX FUV images to identify starforming regions (see Section 4.2), and the global galaxy properties are derived from a combination of the previously published data set as a whole (see Section 6).

\section{GALAXY REGIONS}

To study individual regions inside of a galaxy, the extent of a galaxy's flux needs to first be defined. There are three previously published galaxy apertures defined at UV, optical, and IR wavelengths for the LVL sample. The optical apertures are those from the RC3 catalogue (de Vaucouleurs et al. 1991) and are defined as isophotal ellipses with surface brightness of $25 \mathrm{mag} \mathrm{arcsec}^{-2}$ in the $B$-band filter. The optical apertures are tabulated in Cook et al. (2014a).

The Spitzer Space Telescope IR elliptical apertures of Dale et al. (2009) were chosen to encompass the majority of the emission seen at Spitzer IR $(3.6-160 \mu \mathrm{m})$ wavelengths. In practice, these apertures were usually determined by the extent of the $3.6 \mu \mathrm{m}$ emission given the superior sensitivity of the $3.6 \mu \mathrm{m}$ array coupled with the relatively bright emission from older stellar populations at this wavelength. However, in several instances the emission between $160 \mu \mathrm{m}$ wavelengths were spatially more extended, and thus images at these wavelengths were used to determine the IR apertures. The resulting median ratio of IR-to-optical semimajor axes is 1.5 .

The GALEX UV elliptical apertures of Lee et al. (2011) were defined as an isophotal ellipse outside of which the photometric error was greater than 0.8 mag or the intensity fell below the sky level. The resulting median ratio of UV-to-optical semimajor axes is 2.3 .

A source of scatter in any relationship between LF slope and global galaxy properties is the crowding of sources due to high galaxy inclination angles. Nearly edge-on (high inclination angle) galaxies will contain star-forming regions which are partially, or fully, obscured by the disc of the galaxy, or blended with sources 
along the line of sight. Therefore, we make a cut on inclination angle which is calculated via the equation:

$i=\cos ^{-1}\left(\left[\frac{(b / a)-q_{0}^{2}}{1-q_{0}^{2}}\right]\right)$,

where $i$ is the inclination angle, $a$ and $b$ are the semimajor and minor axis of each galaxy, and $q_{0}$ is the intrinsic axis ratio for an edge-on galaxy set to 0.2 (Tully \& Fisher 1977). We adopt the UV apertures to define the inclination angles and have chosen to exclude galaxies with $i$ greater than $60^{\circ}$ [see also, Prescott et al. (2007)]. However, as a sanity check we compare the inclination angles based on all three apertures (UV, R25, and IR).

The median difference between the inclination angles based on UV apertures and those of the IR and R25 is $10.3 \pm 10.5$ and $0.6 \pm$ 4.1 , respectively. We find 43 galaxies whose inclination angle is cut based on the UV apertures but whose IR or R25 inclination angle is less than $60^{\circ}$. Visual inspection of these galaxies reveals that 22 of them (DDO210, ESO119-G016, NGC2366, NGC2903, NGC2976, NGC3031, NGC3274, NGC3521, NGC3627, NGC4068, NGC4236, NGC4258, NGC4490, UGC01249, UGC04426, UGC05272, UGC05340, UGC05666, UGC06541, UGC06817, UGC07599, and WLM) have morphologies which are irregular and show little evidence of blended sources (e.g. NGC2366) or have spiral morphologies but the entire disc is clearly identifiable (e.g. NGC3031). We verify that our results are not affected if we exclude these galaxies from the analysis. A total of 77 galaxies are excluded from the analysis due to inclination angle.

\section{STAR-FORMING REGIONS}

In this section, we describe the identification, photometry, and extinction correction methods for our star-forming regions as well as the detection limits for each galaxy. We identify star-forming regions based on the GALEX FUV images, which have a full width half maximum (FWHM) of $\sim 5$ arcsec. This resolution corresponds to $\sim 24 \mathrm{pc}$ for the closest galaxy at $\sim 1 \mathrm{Mpc}$ and $\sim 250 \mathrm{pc}$ for the furthest galaxy at $10.5 \mathrm{Mpc}$. Due to the large physical scale of FUV sources at the greatest distances of the LVL sample, the FUV regions identified here can include bound star clusters (a few parsecs), loose association (a few $10 \mathrm{~s}$ of parsecs), or, in some cases, star cluster complexes. However, due to the fractal or scale-free, structure of star formation, we expect that the star-forming regions studied here will exhibit similar distributions as those of the densest parts of the hierarchy, star clusters (see Section 8.1.2).

\subsection{Contaminating source removal}

To prepare the data for identification and photometry, contaminating sources (e.g. background galaxies and foreground stars) were identified and masked. We utilize the contaminant regions identified by Cook et al. (2014a), which are available in all LVL filter bandpasses (i.e. FUV, NUV, UBVR, JHK, IRAC1,2,3,4, MIPS24). A detailed description of the contaminating regions and removal can be found in Cook et al. (2014a), but we provide a brief overview here.

The initial contaminant catalogues were generated by Dale et al. (2009) which were based on Spitzer Space Telescope IR colours and high-resolution hubble space telescope (HST) images. These catalogues were then updated by Cook et al. (2014a) using additional high-resolution $H S T$ images via the Hubble Legacy Archive. ${ }^{1} H S T$ images were visually checked for sources with spiral structure, extended profiles, and optical colours indicating a background galaxy, and sources with diffraction spikes indicating a foreground star. The new contaminant regions were tailored to each image and added to the modified contamination source catalogue of Dale et al. (2009). With an updated contaminating source catalogue, each contaminant was removed through an interpolation of the surrounding local sky using the IRAF task imedit.

\subsection{Source identification}

To ensure that sources in the outer parts of a galaxy are not excluded, we identify star-forming regions inside the aperture with the largest area. In most cases, the UV apertures have the greater area and were subsequently used to define the galaxy's extent. The apertures for each galaxy were visually inspected to verify that no bona fide star-forming regions were found outside the aperture's extent.

The identification of FUV sources was carried out with the publicly available code SExTRACTOR (Bertin \& Arnouts 1996). At the GALEX resolution of 5 arcsec most sources inside the galaxy aperture will be point sources. However, as some sources will be blended or possibly cluster complexes with various morphologies, SEXTRACTOR is ideal to identify these regions since the algorithm locates contiguous pixels above a threshold yielding source identification of any morphology. The SEXTRACTOR background input parameters are set to 128 and 6 for BACKSIZE and BACKFILT, respectively. Other SEXTRACTOR input parameters are determined from tests designed to optimize the extraction of FUV sources in the LVL sample.

Before the detection of pixels above a threshold is performed, we apply a SEXTRACTOR filter (e.g. Gaussian, tophat, mexhat, etc.). To determine the filter best suited to identifying FUV sources in both crowded and uncrowded regions, we run SExTRACTOR on all FUV images using a default (i.e. a pyramidal function), tophat, mexhat, and no filter with the deblending parameters set to their default values.

A graphical representation of SEXTRACTOR identifications can be seen in the segmentation images, where the contiguous pixels of different sources are colour coded. Fig. 1 shows the original FUV image of the dwarf UGC07608 in panel (a) and the segmentation maps for no filter, default, tophat, mexhat filters in panels (b), (c), (d), and (e), respectively. The cyan ellipse in panel (a) represents the UV galaxy aperture defined by Lee et al. (2011) and the green circles represent the SEXTRACTOR identifications using a mexhat filter. The circular apertures have a radius of 3 pixels, which is the size of the photometric aperture (see Section 4.3).

Visual inspection of the segmentation maps for all galaxies shows that using no filter creates contiguous pixels which have boundaries that are ill-defined and have filamentary structure. A typical example is shown in panel (b) of Fig. 1 for UGC07608, where many sources in the galaxy and near the edges of the galaxy show irregular morphologies that sometimes extend in filaments away from the source. This filamentary structure indicates that some of these pixels are fluctuations in the background and can cause centring inaccuracies and boundary confusion with nearby sources.

The default filter segmentation map shown in panel (c) of Fig. 1 shows similar results to using no filter, but with somewhat reduced

\footnotetext{
${ }^{1}$ http://hla.stsci.edu/hlaview.html
} 

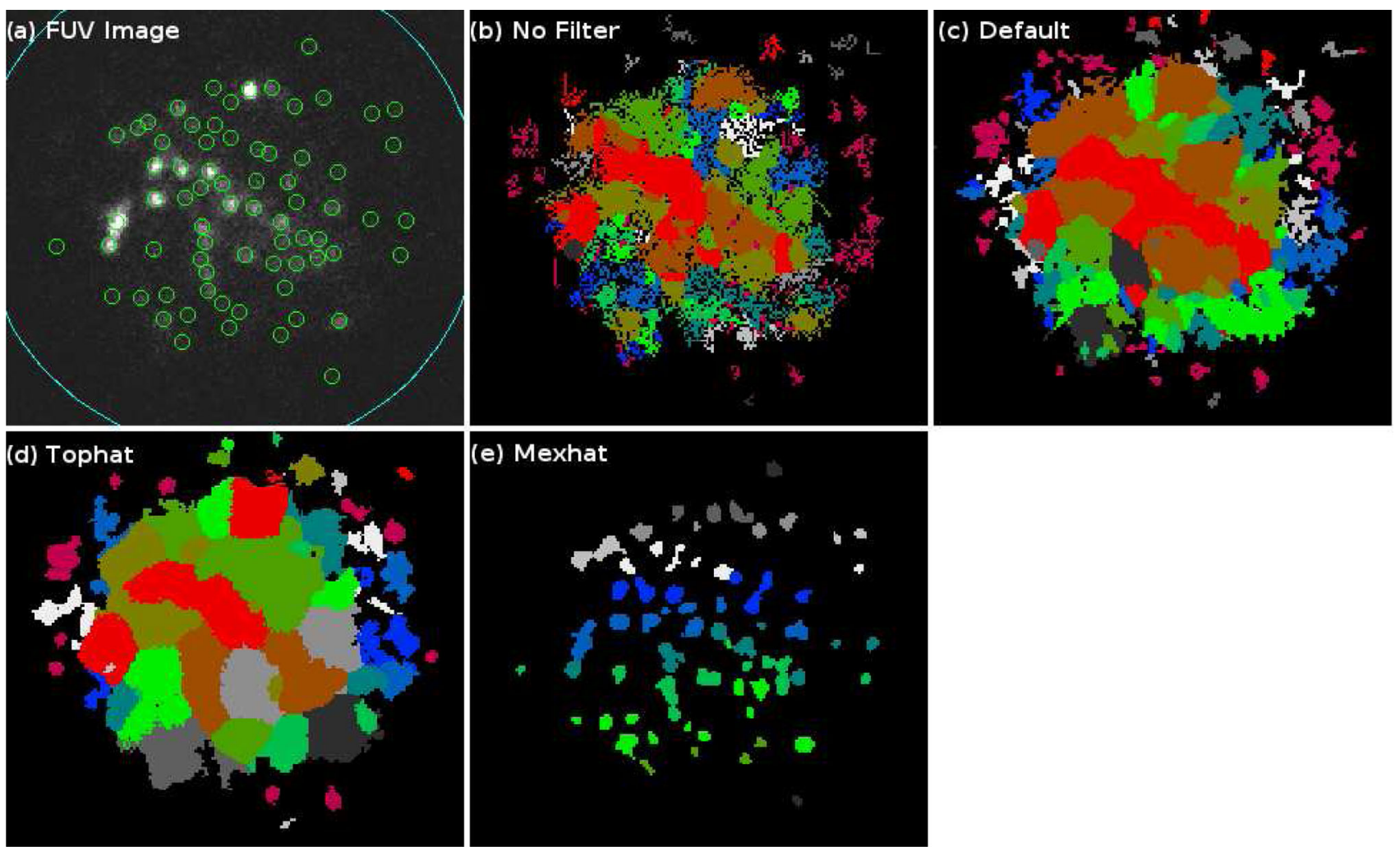

Figure 1. The FUV image and graphical representations of the SEXTRACTOR identifications using different filters for the dwarf galaxy UGC07608. Panel (a) is the GALEX FUV image where the cyan ellipse represents the UV galaxy apertures and the green circles represent the SExTRACTOR identifications using a Mexhat filter. Panels (b)-(e) are the segmentation images using no filter, default (i.e. a pyramidal function), tophat, mexhat filters, respectively. The colours represent a range of integers assigned to each identified source.

filamentary structure. In addition, the contiguous pixels for many of the sources have been extended due to the filtering of neighbouring pixels yielding boundary confusion between neighbouring sources similar to using no filter. The tophat filter shown in panel (d) of Fig. 1 shows fewer spurious faint sources located near the edges of the galaxy, but shows extended contiguous pixels similar to using both no filter and the default filter.

The mexhat filter shown in panel (e) of Fig. 1 shows almost no filamentary structure and well-behaved boundaries (i.e. more round) in the contiguous pixels of most sources. Furthermore, the clear separation in the boundaries between nearby sources will yield more accurate centring information and better deblending of sources in crowded regions. In addition, the mexhat segmentation maps is visually similar to the original FUV image of panel (a) indicating robust identification.

One caveat when using the mexhat filter is that faint sources in the outskirts are sometimes not detected requiring that a $1 \sigma$ threshold be used in order to identify them. This is most likely due to counts being spread into the wings of the point spread function (PSF) when convolved with the filter. Using a $1 \sigma$ detection threshold could result in the identification of some spurious sources, however a $3 \sigma$ detection cut is applied to the FUV source catalogue during the photometry process which uses a local background (see Section 4.3).

Fig. 2 is similar to Fig. 1, but for the spiral galaxy NGC3031 (M81). The panels are the same for those of Fig. 1, however, we have removed the circular apertures for visual clarity. We find similar filtering results for this galaxy, where the mexhat filter contiguous pixels of identified sources show well-behaved boundaries and generally good boundary separation between nearby sources. It is interesting to note that the FUV regions identified with the mexhat filter in panel (e) of Fig. 2 show clear spiral structure mimicking the structure of the original FUV image of panel (a). We use a mexhat filter with a $1 \sigma$ detection threshold for all FUV galaxies; as noted previously, we apply a $3 \sigma$ detection cut on all sources during the photometry process.

We have also added a yellow region in the centre of panel (a) which represents the central $1 \mathrm{kpc}$ region of this galaxy. The nucleus of NGC3031 is clearly separated from the star-forming disc and is likely to be a conglomeration of many star-forming regions. There are similar central regions in the following galaxies: NGC1291, NGC3344, NGC4258, NGC5194, NGC1512, NGC3351, NGC4736, NGC5236, NGC2903, NGC3368, NGC5055, NGC5457, NGC3031, NGC3623, NGC5068, NGC7090. All of the central regions in these galaxies have been visually identified and subsequently removed to avoid contamination of the star-forming region sample [see also, Prescott et al. (2007)].

Blending of nearby sources is likely to occur in our FUV source catalogue due to the relatively large PSF of the FUV images. SExTRACTOR utilizes a deblending algorithm which allows the user to choose the degree of deblending through the parameters DEBLEND_NTHRESH and DEBLEND_MINCONT. To test which parameters are best suited to the LVL FUV images we analyse the SEXTRACTOR outputs using a reasonable range of both DEBLEND_NTHRESH and DEBLEND_MINCONT while keeping the background and filtering parameters the same for each deblending parameter. We also note that galaxies at greater distances may be affected more by blending issues; however, we provide a thorough investigation of these effects in Section 8.1.2 and find 

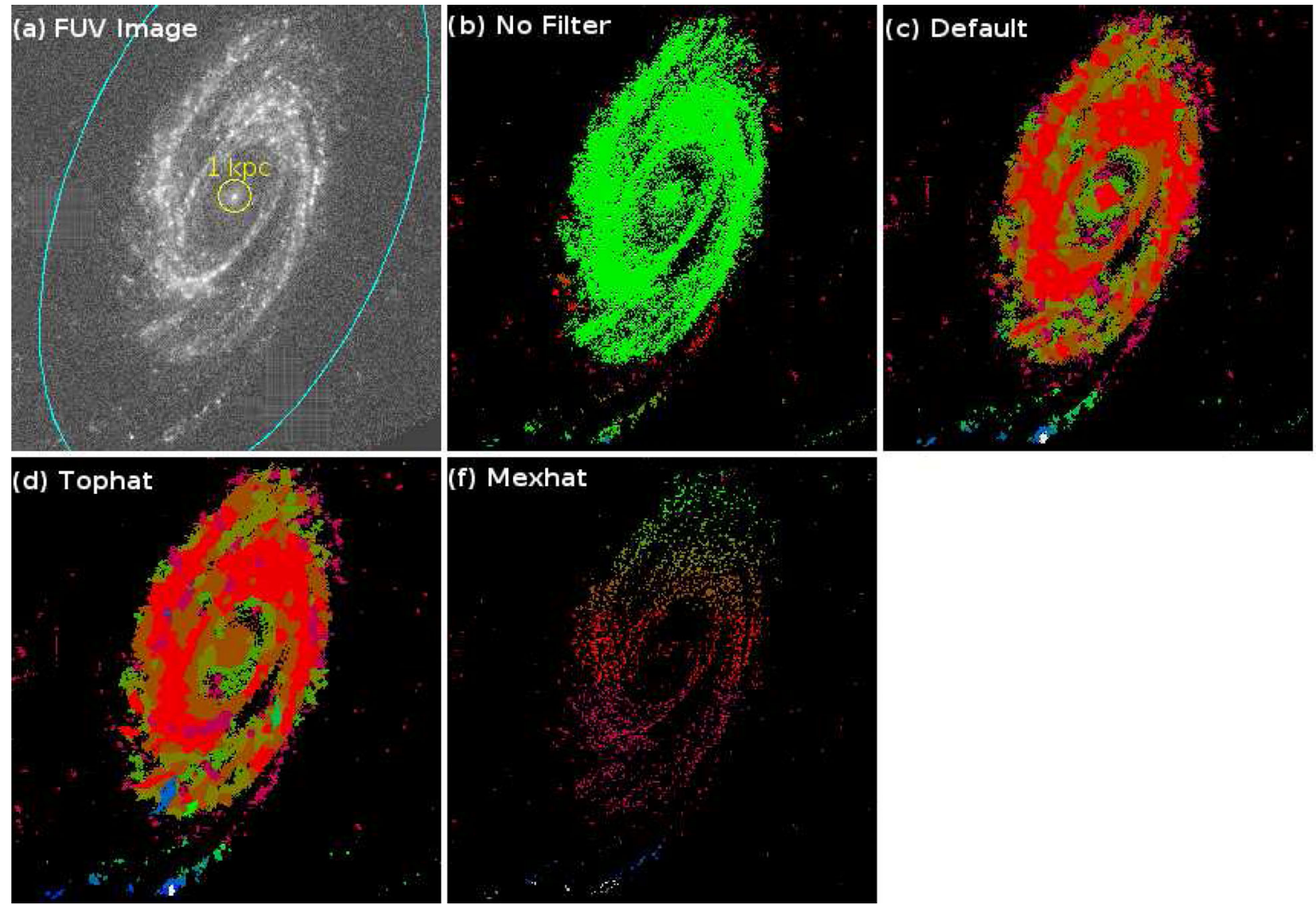

Figure 2. Same as Fig. 1, but for the spiral galaxy NGC3031 (M81). It is interesting to note that the FUV regions identified with the mexhat filter in panel (e) of Fig. 2 show clear spiral structure mimicking the structure of the original FUV image of panel (a).

that blending due to distance does not significantly affect our results.

Since the process of deblending of sources will increase the total number of sources identified, the deblending parameters are optimized by examining the total number of identified sources. We find that the total number of all FUV sources identified in the LVL sample increases nearly asymptotically with the DEBLEND_MINCONT parameter towards a value of $\sim 65000$. The total number plateaus near a DEBLEND_MINCONT value of $1 \mathrm{e}-5$, which is the value we adopt. For each DEBLEND_MINCONT value the maximum number of sources occurs for the DEBLEND_NTHRESH parameter of 64 , which is the value we adopt. The total number of sources found in all of our galaxies is $\sim 65000$.

\subsection{Photometry}

Photometry for FUV sources was carried out via the IRAF task PHOT within an aperture of a 3 pixel (4".5) radius, where the centres are from SEXTRACTOR. Due to the large PSF of the FUV images, many of the sources have contaminating nearby sources. Visual inspection of crowded regions in the LVL sample reveals that an aperture of 3 pixels $(1.8 \times$ FWHM $)$ minimizes contaminating light from nearby sources while capturing the majority of light for the target region. The sky annuls is set to a radius of 7 pixels and a width of 1 pixel with a mode algorithm to minimize local contaminants. Next, we apply an aperture correction to recover the total flux of our star-forming regions.
The aperture corrections for GALEX FUV point sources have been characterized by previous studies (Martin et al. 2005; Morrissey et al. 2007), but the star-forming regions studied here may not be point sources even at the 5 arcsec resolution of the GALEX FUV images. In addition, these previous studies have found that the FHWM of GALEX point sources can change from 4".5 to 6". from image to image and across the FOV of an image. For these reasons we use a training set of isolated FUV sources located inside the galaxies aperture to investigate an appropriate, empirical aperture correction. The techniques used in this study are similar to those used in star cluster studies of higher resolution (e.g. Chandar et al. 2010)

The training set is composed of FUV sources in all galaxies manually selected to have no contaminating flux within a 15 pixel radius. For each training set object we can measure an aperture correction defined as the magnitude difference (i.e. flux ratio) at 15 pixels and 3 pixels radii, where the sky annulus is set to 16 pixels and a width of 1 pixel. A sample-wide aperture correction can be determined by taking the average and standard deviation that can be applied to all sources yielding a correction of $-0.86 \pm 0.34$ mag. Although an average correction is straight forward, it may not reflect an accurate aperture correction for each source; especially if there is a significant range of PSFs or morphologies (i.e. the extent of the radial profile).

We test the dependence of each object's aperture correction on the radial extent by measuring the concentration index (hereafter $\mathrm{CI}$ ) of all training set objects, where we define $\mathrm{CI}$ as the magnitude difference measured at 1 and 3 pixels. The 1 and 3 pixel radii 


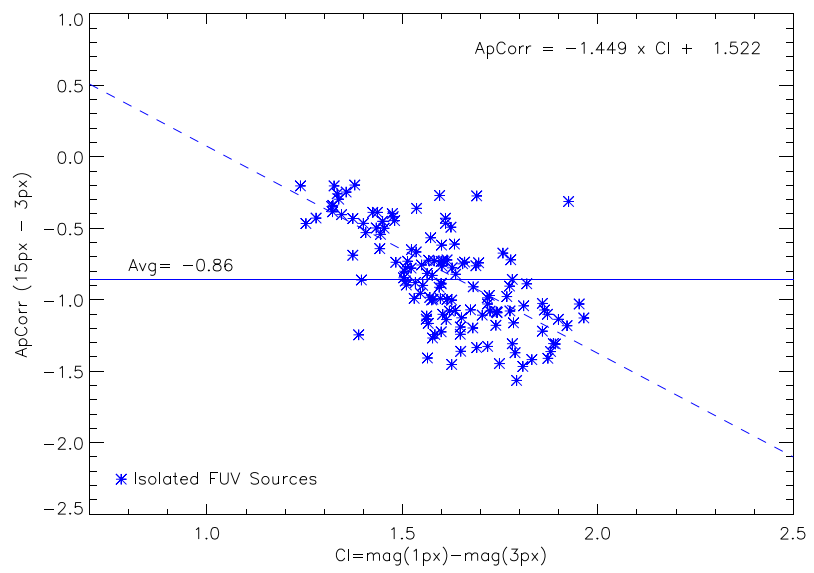

Figure 3. The plot of aperture correction versus concentration index (CI) for isolated FUV sources. The aperture correction is defined as the magnitude difference at 15 and 3 pixel radii (the photometry aperture) and the $\mathrm{CI}$ is defined as the magnitude difference at 1 and 3 pixels. CI is a measure of a source's radial profile extent and the relationship shows that more extended objects require a larger aperture correction.

definition was chosen, since smaller than 1 pixel apertures will have increased photometry uncertainties and since crowding may become an issue for apertures larger than 3 pixels. Previous studies of starforming regions (i.e. star clusters) have used CI to quantify the extent of an object's radial profile and have found relationships between the two quantities (e.g. Chandar et al. 2010). The dependence of aperture correction on CI makes sense since a more extended object (i.e. higher CI) will have more light in the wings compared to a point source (i.e. smaller $\mathrm{CI}$ ) and therefore will have a greater aperture correction.

Fig. 3 shows a relatively strong correlation between aperture correction and CI for the training set FUV sources, where the solid line represents the average aperture correction to all isolated FUV sources and the dashed line represents the fitted relationship between aperture correction and CI. The fitted relationship between aperture correction and $\mathrm{CI}$ is described by the equation:

$\operatorname{ApCorr}(\mathrm{FUV})=-1.45 * C I+1.52$.

Although an average aperture correction is suitable for most studies of star-forming regions, Fig. 3 shows that at the low and high CI extremes of the training set, the CI-based aperture correction can differ from the average aperture correction by as much as $0.6-0.8$ mag. These total flux differences could result in systematic deviations in the LFs of star-forming regions. We use the CI-based aperture corrections to yield the final magnitudes of our star-forming regions and to analyse the relationships between LF slope and galaxy properties. However, we have verified that using an average aperture correction does not significantly change our results; although the LF slope trends with galaxy properties do show some increased scatter compared to the results using the CI-based aperture corrections.

Since the light from an FUV source will be affected by attenuation due to dust we additionally match any $24 \mu \mathrm{m}$ sources to our FUV sources, which will be used to account for this attenuated light (see Section 4.4). Photometry is performed on any $24 \mu \mathrm{m}$ matched source using the same photometry parameters used for the FUV sources: 3 pixel aperture, 7 pixel sky and 1 pixel width sky annuls. Although the FWHM for $24 \mu \mathrm{m}$ and FUV point sources is similar, we derive an empirical aperture correction for $24 \mu \mathrm{m}$ sources using

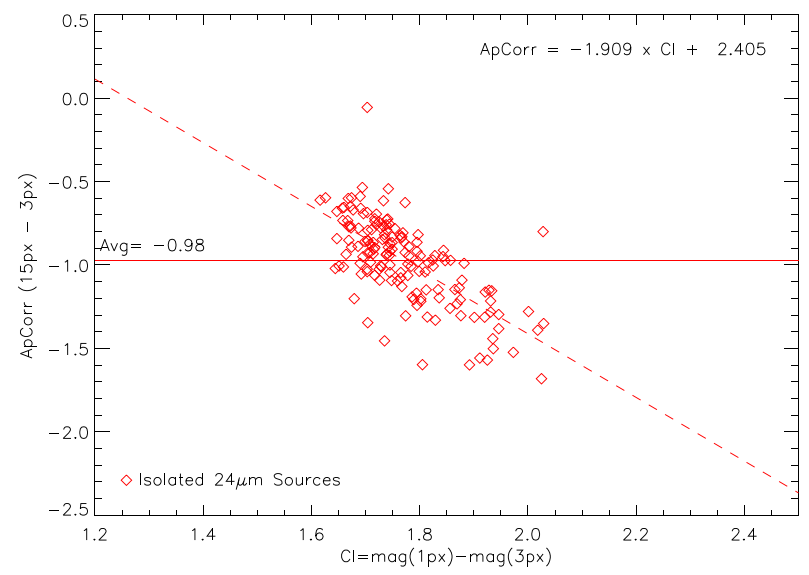

Figure 4. The plot of aperture correction versus concentration index for isolated $24 \mu \mathrm{m}$ sources. The relationship shows that more extended objects require a larger aperture correction.

the same methods for FUV sources. Fig. 4 shows the relationship between aperture correction and CI for MIPS $24 \mu \mathrm{m}$ sources.

We select isolated $24 \mu \mathrm{m}$ sources inside each galaxy's aperture and require them to have an FUV counterpart. An aperture correction $[\operatorname{mag}(15$ pixels $)-\operatorname{mag}(3$ pixels $)]$ and CI $[\operatorname{mag}(1$ pixels $)-$ mag(3 pixels)- are measured for each isolated source and plotted in Fig. 3. We find a strong correlation between aperture correction and CI which is described by the equation:

$\operatorname{ApCorr}(24 \mu \mathrm{m})=-1.91 * C I+2.41$.

Similar to FUV sources, the difference between the CI-based aperture corrections for $24 \mu \mathrm{m}$ can differ from the average aperture correction by as much $0.5-0.7 \mathrm{mag}$. Since this difference is not insignificant, we use the CI-based aperture corrections to measure the total flux for $24 \mu \mathrm{m}$ sources.

The detection limits for the FUV images are defined here as the $5 \sigma$ detection limit for a point source with a FWHM of 5 arcsec. The standard deviation in the sky was measured within equal area regions distributed in an annuli around the galaxy at a distance of 24 times the D_25 radius. The resulting average $5 \sigma$ detection limit for the FUV images is $\log L\left(\mathrm{erg} \mathrm{s}^{-1}\right)=38.0$ with a standard deviation of 0.83 , and the corresponding $\mathrm{AB}$ magnitude average limit is 21.6 $\pm 1.3 \mathrm{mag}$. We express the luminosities for individual regions in our LFs as $d \nu L_{v}$ integrated under the filter curve of the FUV filter $(\lambda=$ $1516 \AA$ and $\mathrm{d} \lambda=268 \AA$ ); note that a monochromatic luminosity (i.e. $v L_{v}$ ) can be recovered with a 0.75 dex shift in $\log$ luminosity.

\subsection{Dust correction of star-forming regions}

To account for FUV flux which has been absorbed by dust and re-radiated into IR wavelengths, we apply a dust correction to each FUV identified star-forming region based on the $24 \mu \mathrm{m} / F U V$ luminosity ratio. There are several previous studies that have derived a relationship between extinction at FUV wavelengths $\left(A_{\mathrm{FUV}}\right)$ and an IR(TIR, $24 \mu \mathrm{m}$, etc.)/FUV luminosity ratio for both galaxies (e.g. Buat et al. 2005; Hao et al. 2011) and regions within galaxies (e.g. Leroy et al. 2008; Liu et al. 2011). However, we derive our own dust correction prescription from panchromatic data of individual star-forming regions where $A_{\mathrm{FUV}}$ is based on a direct measure of dust attenuation (i.e. Hydrogen recombination lines).

We derive a dust correction prescription based on the FUV, $24 \mu \mathrm{m}$, $\mathrm{Pa} \alpha$, and $\mathrm{H} \alpha$ luminosities of individual star-forming regions in 
M51 published by Calzetti et al. (2005), where the FUV and $24 \mu \mathrm{m}$ luminosities are expressed as $v L_{v}$ and the nebular line luminosities are expressed as $d \nu L_{\nu}$ integrated under the filter curve [see Calzetti et al. (2005)]. We convert our FUV and $24 \mu \mathrm{m}$ luminosities to monochromatic luminosities $\left(v L_{v}\right)$ when calculating dust corrections. Since the $\mathrm{Pa} \alpha / \mathrm{H} \alpha$ luminosity ratio is a direct measure of the flux absorbed by dust, we have derived an empirical relationship between $24 \mu \mathrm{m} / \mathrm{FUV}$ luminosity ratio and $A_{\mathrm{FUV}}$ based on the $\mathrm{Pa} \alpha / \mathrm{H} \alpha$ luminosity ratio for individual star-forming regions.

Assuming case $\mathrm{B}$ recombination, the $E(B-V)$ value for each region can be derived via the relationship between the nebular emission line colour excess and the luminosity ratio of $\mathrm{Pa} \alpha$ and $\mathrm{H} \alpha$ (see the appendix in Momcheva et al. 2013, for details):

$$
\begin{aligned}
E(B-V) & =\frac{E(H \alpha-P a \alpha)}{k\left(\lambda_{H \alpha}\right)-k\left(\lambda_{P a \alpha}\right)} \\
& =\frac{2.5}{k\left(\lambda_{H \alpha}\right)-k\left(\lambda_{P a \alpha}\right)} \log _{10}\left(\frac{L(P a \alpha / H \alpha)_{\mathrm{obs}}}{L(P a \alpha / H \alpha)_{\text {int }}}\right),
\end{aligned}
$$

where $k(\lambda)$ is the extinction curve values $\left(k\left(\lambda_{H \alpha}\right)=2.535\right.$ and $k\left(\lambda_{P a \alpha}\right)=0.45$; Cardelli, Clayton \& Mathis 1989) evaluated at the given wavelength, $L(P a \alpha / H \alpha)_{i n t}$ is the intrinsic luminosity ratio of 0.128 (Osterbrock \& Ferland 2006), and $L(P a \alpha / H \alpha)_{\mathrm{obs}}$ is the observed luminosity ratio of $\mathrm{Pa} \alpha$ and $\mathrm{H} \alpha$ in units of ergs s${ }^{-1}$. $A_{\mathrm{FUV}}$ can then be calculated given the equation:

$A_{\lambda}=k(\lambda) E(B-V)^{\prime}$,

where we have adopted the Calzetti et al. (2000) extinction curve value of 10.27 for $k\left(\lambda_{\mathrm{FUV}}\right)$. We have also included a factor of 0.44 in $E(B-V)$ to convert the emission line attenuation (i.e. Hydrogen recombination lines) to stellar continuum attenuation (Calzetti 2001), where the stellar continuum colour excess is represented by $E(B-V)^{\prime}$.

The top panel of Fig. 5 shows a correlation between $A_{\mathrm{FUV}}$ and the $24 \mu \mathrm{m} / \mathrm{FUV}$ luminosity ratios of 42 regions in Calzetti et al. (2005). The solid line is the bisector fit to the data which is described by the equation:

$A_{\mathrm{FUV}}=1.46 \times \log _{10}\left(\frac{L(24 \mu \mathrm{m})}{L(\mathrm{FUV})}\right)+3.09$,

where the luminosities are in units of $\mathrm{erg} \mathrm{s}^{-1}$ and $A_{\mathrm{FUV}}$ is in $\mathrm{AB}$ magnitude units.

The blue-dashed line in Fig. 5 represents the galaxy-wide dust correction relation of Hao et al. (2011). We note that the data for individual regions lies above the galaxy-wide dust correction line of Hao et al. (2011) indicating that the dust attenuation is greater than those of derived for galaxies. It is not likely that galaxy-wide dust corrections are applicable to individual star-forming regions (Calzetti et al. 2005; Boquien et al. 2015), and this increased dust attenuation for individual regions has been observed by previous authors (e.g. Calzetti et al. 2005, 2007; Leroy et al. 2008; Liu et al. 2011).

The red-dotted line in Fig. 5 represents a previously derived dust correction for individual regions (Leroy et al. 2008; Liu et al. 2011). We find that the previous dust correction prescription for individual regions shows better agreement with the M51 data compared to the galaxy-wide prescription, but also falls below the majority of the M51 data points. The dust correction prescription derived in this study (the bisector fit) shows better agreement with the M51 data. Consequently, we use the bisector fit to correct the FUV luminosities

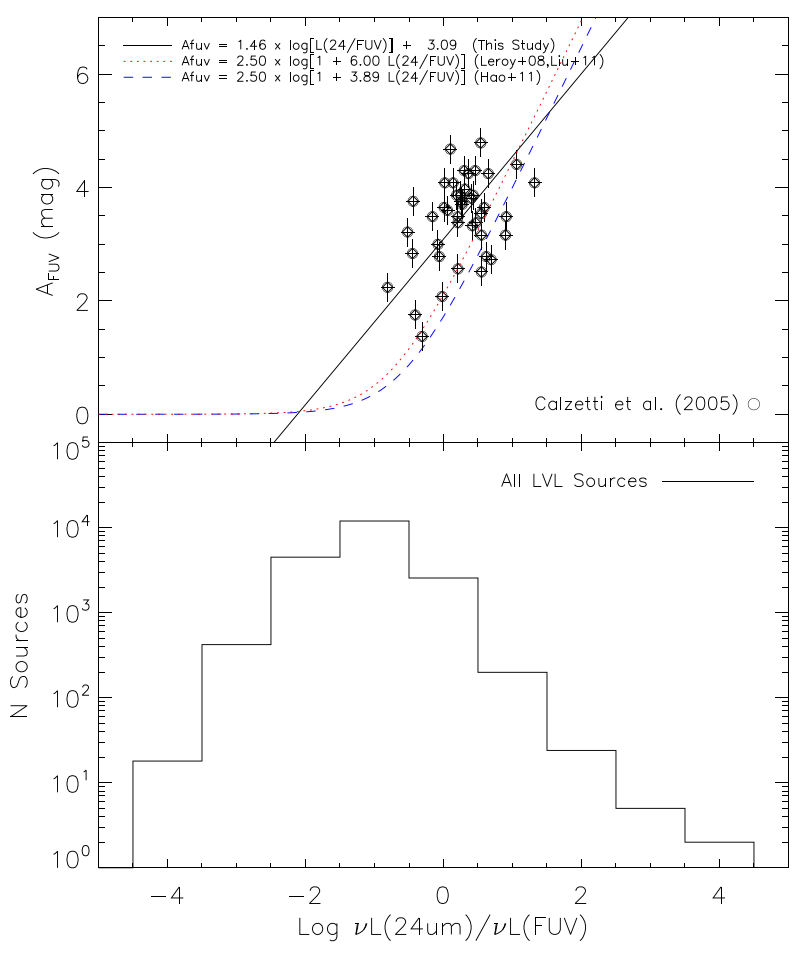

Figure 5. Top panel: the plot of extinction in the FUV bandpass ( $\left.A_{\mathrm{FUV}}\right)$ versus the logarithmic ratio of $24 \mu \mathrm{m} / \mathrm{FUV}$ luminosities for the star-forming regions of Calzetti et al. (2005) in M51. The solid black line is the bisector fit to the data of Calzetti et al. (2005), the blue-dashed line is the relationship prescribed by Hao et al. (2011) for galaxies, and the red-dashed line is the relationship prescribed by Leroy et al. (2008) and Liu et al. (2011) for individual regions. We use the relationship derived from the star-forming regions of Calzetti et al. (2005) to correct our FUV sources for flux attenuated by dust. Bottom panel: the histogram of the $24 \mu \mathrm{m} / \mathrm{FUV}$ luminosity ratios for all identified star-forming regions in our study.

of our star-forming regions. In addition, we verify that all three dust correction prescriptions provide similar trends between LF slope and galaxy properties (see Section 7.2).

It could be argued that the functional form of the previous dust correction prescriptions (Leroy et al. 2008; Hao et al. 2011; Liu et al. 2011) might better reflect the relationship between dust attenuation and attenuation indicators (i.e. UV/IR luminosity ratios). We perform a fit with this functional form to the M51 data and obtain similar results to those of Leroy et al. (2008) and Liu et al. (2011), where our fitted slope is slightly higher than those of the previous prescriptions. We also note that there is almost no difference in the trends between LF slope and galaxy properties when using the Leroy et al. (2008) and Liu et al. (2011) dust prescription and a dust prescription derived from M51 data using the functional form of previous studies.

The bottom panel of Fig. 5 shows the $24 \mu \mathrm{m} / \mathrm{FUV}$ luminosity ratio distribution for all star-forming regions identified in this study. We note that $A_{\mathrm{FUV}}$ values will become negative for regions with $24 \mu \mathrm{m} /$ FUV luminosity ratios less than $\sim-2$. To prevent negative dust corrections, we set any negative $A_{\mathrm{FUV}}$ values to zero.

\section{LUMINOSITY FUNCTIONS}

In this section, we present the LFs for our star-forming regions. We describe the methods used to generate the LFs and the methods used to derive the fitted power-law slopes. In addition, we provide 
LF examples representative of the different types of galaxies in our sample. Given the results of our LF binning tests, we define our logarithmic luminosity bins via an equal number of sources in each bin where the bin centre is the midpoint of the bins.

\subsection{Fitting and binning methods}

Previous studies of star clusters and $\mathrm{H}$ II regions have found that the LFs are adequately approximated by a power law $(\mathrm{d} N / \mathrm{d} L \propto$ $L^{\alpha}$ ), which can be characterized by the exponent $\alpha$. Our LFs are generated by dividing the luminosities of the FUV sources into luminosity bins and counting the number of sources in each bin. A power law is represented as a straight line in logarithmic space, and we subsequently use $\chi^{2}$ minimization to fit a line to our LFs in logarithmic space. The fitted slope of the line is taken as the power-law exponent $(\alpha)$ where the errors in each bin represent the Poisson noise of the sources in the bin and the error in the fitted slope is taken as the error in $\alpha$.

To derive accurate $\alpha$ values, we fit a power law to luminosity bins which are reasonably complete, in other words, in bins that are not missing star-forming regions due to the depth of the observations. A simple measure of the luminosity at which our sampling is reasonably complete is the peak of the luminosity histogram. Since star-forming regions are represented as a power law, the number of regions will continue to increase at lower luminosities. If the LF of star-forming regions is accurately described by a power law with a single slope, then a drop in the number of regions for bins at fainter luminosities (i.e. fainter than the peak of the luminosity histogram) will indicate where completeness begins to affect the shape of the LF. We note that the peak of the luminosity histogram is only a rough estimation of the completeness limit for each galaxy, but we believe that it does provide a reasonable limit to the luminosity bins used in our LF power-law fits (see Appendix A for a visual representation of all our LFs where the peak of the LF is the dashed-line in the LF for each galaxy).

There are different methods to populate sources into each luminosity bin and the choice of the binning method can change the slope of the fitted LF (Maíz Apellániz \& Úbeda 2005). We quantify the effect of binning by deriving LFs using two common techniques: equal luminosity-size bins where each bin has the same luminosity width and equal-number bins where each bin is populated with an equal number of sources. A comparison of $\alpha$ between these two methods serves as a check on the robustness of our LF results.

The equal luminosity-size bins are most commonly used when fitting a power law to the LFs of star-forming regions. However, since bright star-forming regions are relatively rare, especially in low-mass galaxies which dominate the LVL sample, we expect that the brightest luminosity bins will suffer from systematic biases due to low-number statistics. Maíz Apellániz \& Úbeda (2005) showed that derived power-law slopes from equal luminosity-size bins are correlated with the number of sources per bin. Furthermore, Maíz Apellániz \& Úbeda (2005) showed that variable luminosity-size bins, where each bin is populated with an equal number of sources, show a reduced $\alpha$ dependence on the number of sources per bin.

Fig. 6 shows the histogram of $\alpha$ values for both binning methods, where the grey shaded histogram represents the LF slopes using equal-number bins and the red diagonal-lined histogram represent slopes using equal luminosity-size bins. The equal luminosity-size binned slopes tend to have flatter slopes compared to the equalnumber bins. This is in agreement with the results from Maíz Apellániz \& Úbeda (2005), where they found flatter slopes in simulations with lower numbers of sources per bin. We utilize the

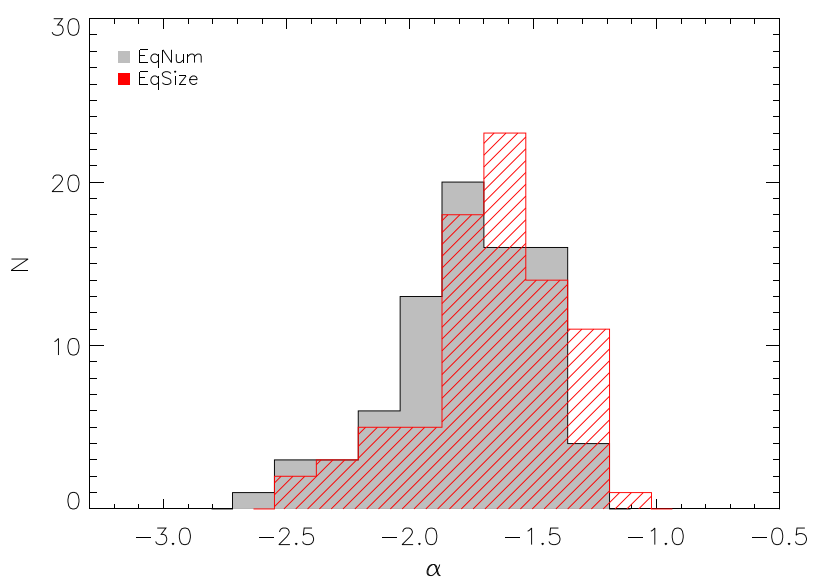

Figure 6. The histogram of luminosity function slopes for two different luminosity binning methods. The grey-shaded histogram represents the LF slopes using equal-number bins and the red diagonal-lined histogram represents slopes using equal luminosity-size bins. The systematic offset of $\alpha$ for equal luminosity-size bins towards flatter slopes is caused by low-number statistics per bin.

LF slopes generated with equal-number bins when examining any trends with global galaxy properties. However, we verify that the results of both binning methods yield similar overall trends between LF slope and global galaxy properties (see Section 7.2).

The difference in LF slopes between equal-number bins and equal luminosity-size bins show an average of -0.21 (equal-number minus equal luminosity-size bins) with a standard deviation of 0.30 . The small average $\alpha$ differences compared to the full range of derived $\alpha$ values $(-2.8<\alpha<-1.0)$ provides some measure of confidence in our derived LF slopes.

In addition to the choice of binning method, we investigate the effect of bin centre definition on LF slopes which could also introduce a bias into the LF shape. For example, if the majority of sources in a bin have luminosities that fall on one side of the bin, then an average of the luminosities would result in a bin centre that is skewed to one side of the bin compared to the midpoint of the bin. The two bin centre definitions we test are: (1) the average of logarithmic luminosities for sources in the bin and (2) the midpoint between the minimum and maximum of logarithmic source luminosities.

To test the effects of bin centre definition, we simulate the LFs for our galaxies based on the real number of sources identified in each galaxy. We assume a universal LF that can be described by a power law with a slope of -2 . For each galaxy, we randomly draw star-forming regions equal to the number of sources found in the galaxy and limit these sources to luminosities above the peak in the real luminosity histogram and below the luminosity of the brightest star-forming region found in the entire sample. The lower luminosity limit mimics the sensitivity of the FUV image, and the upper limit allows for the possibility of creating random bright objects (see Section 8.1.1). The subsequent LF is then fit with the same methods used to fit our real LFs and we iterate 1000 times.

Fig. 7 is a plot of the resulting simulated LF slopes versus the input number of sources for each galaxy. The blue X's represent the median of all simulated $\alpha$ values for average luminosity bin centres, the black diamonds represent the median of all simulated $\alpha$ values for midpoint bin centres, and the error bars represent the 68th and 32 nd percentile of the simulations (i.e. the $1 \sigma$ confidence interval).

Visual inspection of Fig. 7 shows that the average bin centre definition is biased to steeper slopes and have higher errors in each 


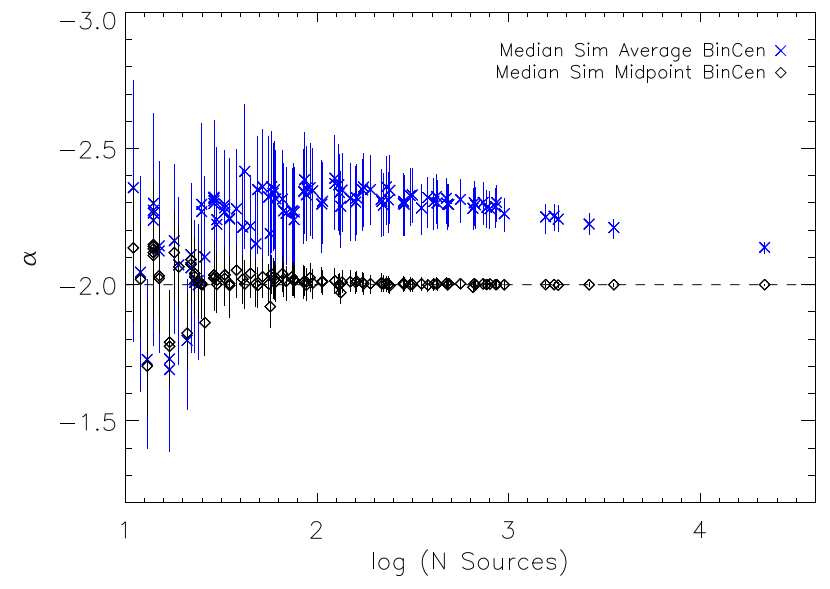

Figure 7. The plot of simulated LF slopes for two bin centre definitions, where blue asterisks represent the average of logarithmic luminosities in each bin and the black diamonds represent the midpoint of the logarithmic luminosities in each bin. The average bin centre definition results in a bias towards steeper LF slopes compared to the input -2 LF slope, while the midpoint bin centre definitions recover the input $-2 \mathrm{LF}$ slope. We use the midpoint bin centre definition to construct our LFs. simulation, while the midpoint centring reproduces the input slope of -2 with less scatter and lower errors per simulated galaxy. In addition, these simulations also show that both bin centring methods show increased random scatter (above and below the input slope of -2 ) towards lower numbers of sources. We address this increased random scatter in Section 8.1.1, and ultimately discard the LFs for galaxies with sources less than 30 (see the next section). For the rest of this study we adopt a fiducial LF binning of equal number sources in each bin where the bin centre is defined as the midpoint of the bin.

We also acknowledge that using a maximum likelihood fitting procedure to determine LF slopes would alleviate many of the complicating effects of binning discussed in this section [see Whitmore et al. (2014)]. However, Whitmore et al. (2014) found good agreement for LF slopes when using similar methods to those used here and a maximum likelihood method. We will provide a comparison to a maximum likelihood method in a future work.

\subsection{Examples}

Fig. 8 shows the LFs and luminosity histogram examples for three representative galaxies with high $(N \sim 1000 \mathrm{~s})$, moderate $(N \sim$ $100 \mathrm{~s})$, and low $(N \sim 10 \mathrm{~s})$ total number of sources. The top panels of Fig. 8 show the LF with equal-number bins and the bottom panels

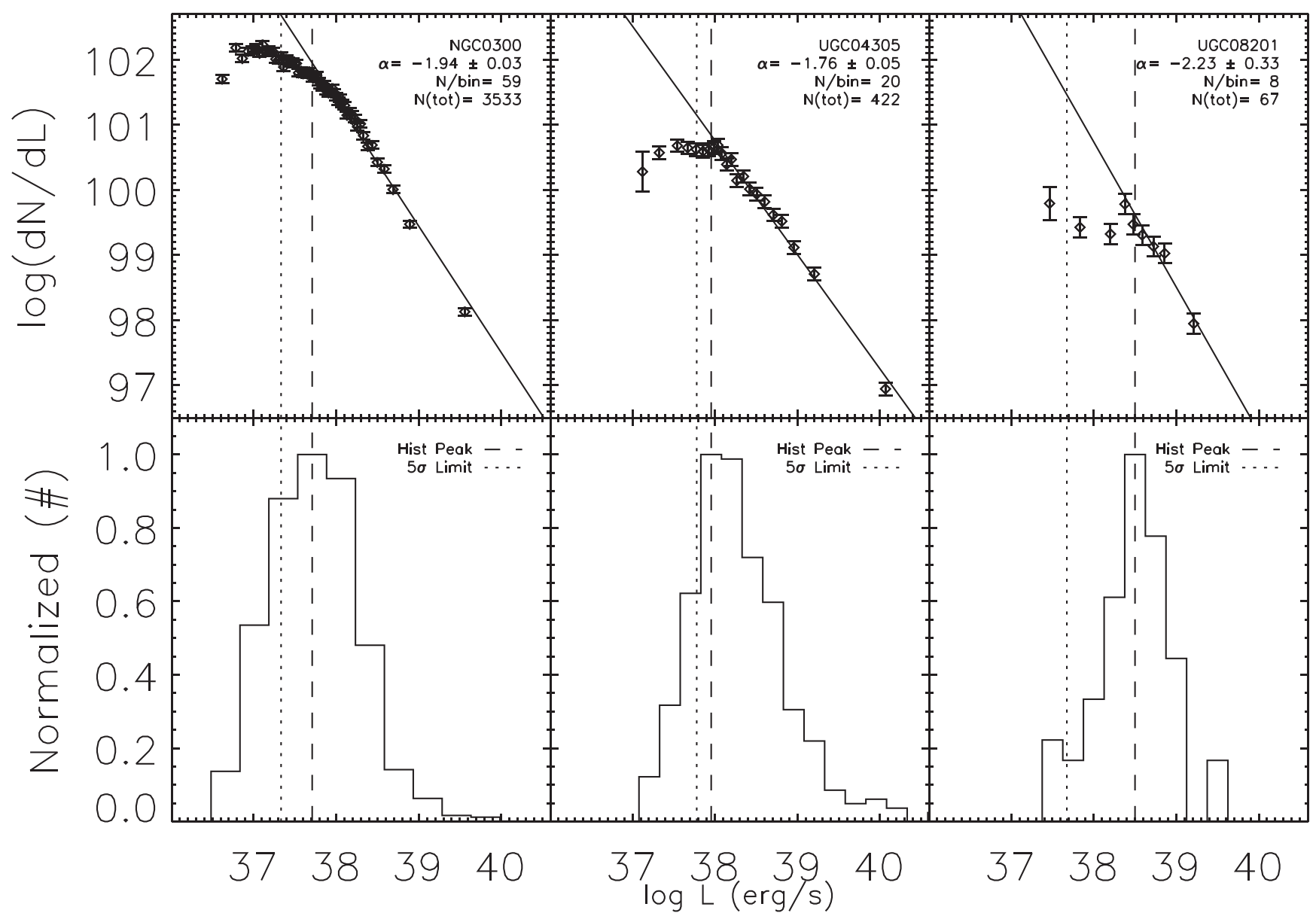

Figure 8. Top panels: example luminosity functions of galaxies with high, moderate, and low numbers of star-forming regions from left to right, respectively. The dotted line represents the $5 \sigma$ detection limit for a point source, and the dashed line represents the peak of the luminosity histogram in the bottom panel. The solid line represents the power-law fit to the LF and extends down to the dashed line. The $y$-axes have been normalized to an arbitrary number. Bottom panels: luminosity histograms for each galaxy in the top panel. The peak in the luminosity histogram accurately traces the LF turnovers in the top panels, and hence, where the effects of incompleteness become significant. 


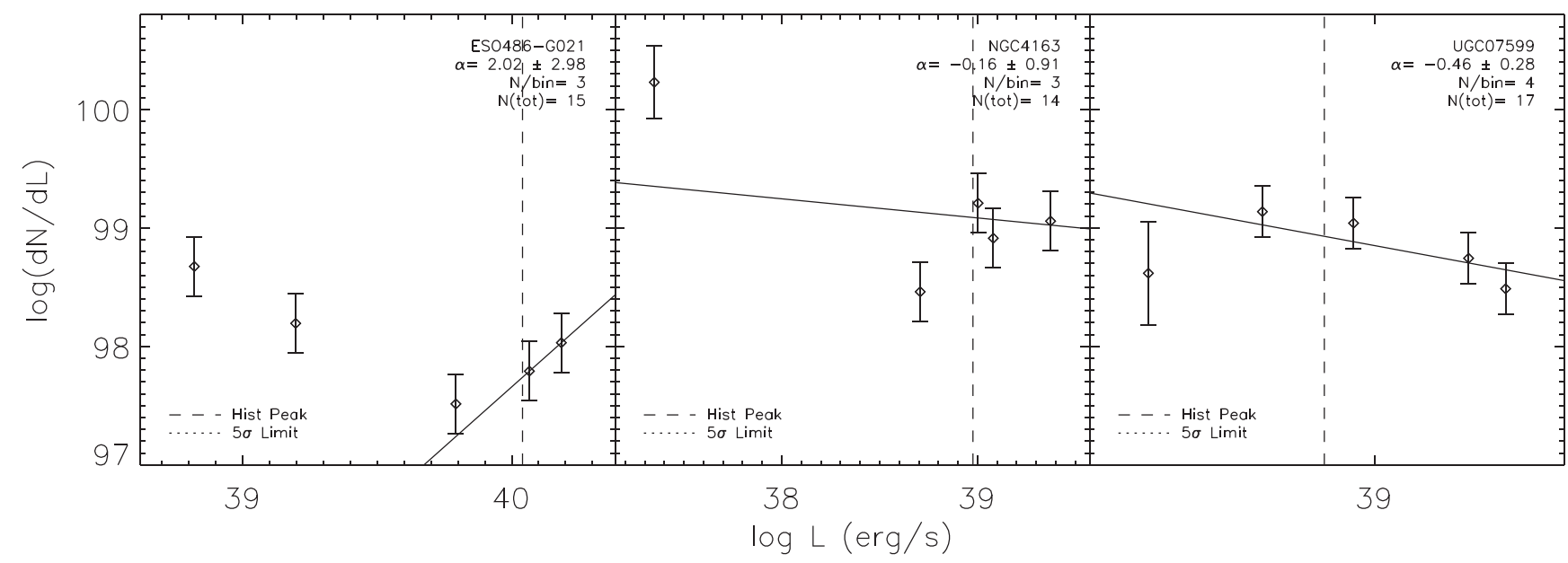

Figure 9. Examples of ill-behaved luminosity functions similar to the top panels of Fig. 8. From left to right, these examples show LFs where: log (dN/d $L$ ) values do not rise continuously towards fainter luminosity bins, the fit depends on only two or three data points, and the data are indistinguishable from a scatter plot. There are 11 galaxies whose LFs are similar to one or more of these examples. The $y$-axes have been normalized to an arbitrary number.

show the luminosity histograms for each galaxy. Visual inspection of the top and bottom panel for each galaxy shows that the peak of the luminosity histogram accurately traces the LF turnover, and hence, where the effects of incompleteness become significant. We find that the peak of the luminosity histogram accurately traces incompleteness effects for all well-behaved LFs.

We define well-behaved LFs as those with $\log (\mathrm{d} N / \mathrm{d} L)$ values which smoothly increase towards lower luminosity bins and either level off or decrease at luminosities fainter than the peak of the luminosity histogram (see Fig. 8). Conversely, we define illbehaved LFs as those satisfying any of the following three conditions: (1) the $\log (\mathrm{d} N / \mathrm{d} L)$ values do not rise smoothly, or at all, towards lower luminosities; (2) the fit depends on only two or three luminosity bins and there exists a vertical discontinuity between any of the luminosity bins; and (3) The LF is extremely noisy and the plot is indistinguishable from a scatter plot. There are 11 galaxies whose LFs are not well behaved (ESO486G021, NGC3299, NGC4163, NGC4190, NGC5238, UGC06900, UGC07599, UGC07605, UGC07950, UGC09992, UGCA438), and we remove these galaxies from the analysis.

Fig. 9 shows three ill-behaved LFs which exemplify the three conditions defined in the previous paragraph. Panel (a) shows a LF whose $\log (\mathrm{d} N / \mathrm{d} L)$ values do not rise continuously towards fainter luminosity bins; in fact, the $\log (\mathrm{d} N / \mathrm{d} L)$ values decease for the first three luminosity bins. Panel (b) of Fig. 9 shows a LF whose log $(\mathrm{d} N / \mathrm{d} L)$ values do increase continuously towards the peak of the luminosity histogram, but the fit depends on only three data points. Furthermore, the large jump in the next two $\log (\mathrm{d} N / \mathrm{d} L)$ values after the peak (aka discontinuity) indicates that the fit to the brightest luminosity bins are relatively uncertain. Panel (c) of Fig. 9 shows a $L F$ whose $\log (\mathrm{d} N / \mathrm{d} L)$ values do increase continuously towards the peak of the luminosity histogram, however, the fit depends on only three luminosity bins and the data are indistinguishable from a scatter plot.

All galaxies with ill-defined LFs have similar overall structure to one or more of the LF examples of Fig. 9. In addition, they all share similar galaxy morphologies and a low total number of sources. The FUV images of these galaxies generally show a 'blobby' morphology where the galaxy has a few central blended sources and a few surrounding faint sources. Furthermore, these galaxies generally have a low total number of sources, where the largest number of sources in a galaxy with an ill-behaved LF is 24 sources. To avoid contaminating our results with unreliable LFs, we exclude any galaxy with a total number of sources $N<30$.

After removing 99 galaxies $N<30$ star-forming regions (which include those with ill-behaved LFs) and 77 galaxies with $i \geq 60$ (except those that have been manually added back in; see Section 3 ) we have a final galaxy sample of 82 galaxies. Figs A1-A10 show the LFs for all 82 galaxies. The LF properties for these galaxies are tabulated in Table 2, where we include the total number of sources, the peak in the luminosity histogram, the luminosity of the brightest source, and the fitted slope $\alpha$ using four combinations of two aperture correction methods and the LF binning methods in each galaxy.

Visual inspection of the LFs in Figs A1-A10 shows a continuous rise in $\log (\mathrm{d} N / \mathrm{d} L)$ values towards fainter luminosity bins and a turnover near the peak in the luminosity histogram where we expect a reduced number of regions due to incompleteness effects. In addition, we find no clear evidence for a break in any individual LF. We use the fitted power-law parameters to investigate any possible relationships between LFs and global galaxy properties.

\section{GLOBAL GALAXY PROPERTIES}

In this section, we describe the global galaxy properties of the LVL sample. The properties presented here were derived in Cook et al. (2014b), but we provide a brief overview and any relevant caveats.

\subsection{Global dust correction}

The internal dust corrections for the majority of the galaxies are carried out via the prescription of Hao et al. (2011) where the extinction in the FUV bandpass $\left(A_{\mathrm{FUV}}\right)$ is first calculated via the empirical relationship with the $24 \mu \mathrm{m}$-to-FUV luminosity ratio $\left(L_{24} / L_{\mathrm{FUV}}\right)$. All other bandpass extinctions $\left(A_{\lambda}\right)$ are derived from $A_{\mathrm{FUV}}$ in combination with the dust extinction curve of Draine (2003).

However, not all galaxies have $24 \mu \mathrm{m}$ and/or FUV detections $(N=47)$ from which to derive internal extinctions. For these galaxies we calculate $A_{\mathrm{H} \alpha}$ based on the empirical scaling relationship from Lee et al. (2009) between $A_{\mathrm{H} \alpha}$ and $M_{B}$, where the $A_{\mathrm{H} \alpha}$ values are derived from spectroscopic Balmer decrement measurements. We derive all other bandpass extinctions for these 47 galaxies using 
Table 2. Luminosity function properties.

\begin{tabular}{|c|c|c|c|c|c|c|c|}
\hline $\begin{array}{l}\text { Galaxy } \\
\text { Name } \\
\text { (1) }\end{array}$ & $\begin{array}{l}\mathrm{N}_{\text {srcs }} \\
\text { (No.) } \\
(2)\end{array}$ & $\begin{array}{c}\log \left(L_{\max }\right) \\
\left(\operatorname{erg~s}^{-1}\right) \\
(3)\end{array}$ & $\begin{array}{c}\log (\text { Lum Peak }) \\
\left(\operatorname{erg~s}^{-1}\right) \\
(4)\end{array}$ & $\begin{array}{c}\alpha \\
\text { EqNum-CI } \\
\quad(5)\end{array}$ & $\begin{array}{c}\alpha \\
\text { EqNum-Avg } \\
(6)\end{array}$ & $\begin{array}{c}\alpha \\
\text { EqSize-CI } \\
\quad(7)\end{array}$ & $\begin{array}{c}\alpha \\
\text { EqSize-Avg } \\
(8)\end{array}$ \\
\hline ESO119-G016 & 30 & 40.09 & 39.33 & $-1.37 \pm 0.41$ & $-1.45 \pm 0.40$ & $-1.59 \pm 0.60$ & $-1.55 \pm 0.40$ \\
\hline ESO245-G005 & 130 & 39.95 & 38.56 & $-1.62 \pm 0.16$ & $-1.71 \pm 0.18$ & $-1.61 \pm 0.17$ & $-1.81 \pm 0.21$ \\
\hline ESO245-G007 & 48 & 36.82 & 36.22 & $-1.61 \pm 0.40$ & $-2.39 \pm 0.73$ & $-1.27 \pm 0.60$ & $-2.02 \pm 0.64$ \\
\hline IC5152 & 150 & 39.99 & 38.53 & $-1.77 \pm 0.16$ & $-1.91 \pm 0.16$ & $-1.74 \pm 0.18$ & $-1.79 \pm 0.17$ \\
\hline IC5332 & 652 & 41.18 & 39.32 & $-1.92 \pm 0.07$ & $-1.87 \pm 0.06$ & $-1.88 \pm 0.08$ & $-1.78 \pm 0.06$ \\
\hline NGC0045 & 478 & 40.67 & 38.70 & $-1.55 \pm 0.06$ & $-1.81 \pm 0.09$ & $-1.49 \pm 0.06$ & $-1.75 \pm 0.09$ \\
\hline NGC0300 & 3533 & 40.08 & 37.71 & $-1.94 \pm 0.03$ & $-2.21 \pm 0.03$ & $-2.05 \pm 0.03$ & $-2.18 \pm 0.04$ \\
\hline NGC0598 & 21617 & 40.04 & 36.87 & $-1.75 \pm 0.01$ & $-1.98 \pm 0.01$ & $-1.70 \pm 0.01$ & $-1.93 \pm 0.01$ \\
\hline NGC0628 & 795 & 41.65 & 38.78 & $-1.46 \pm 0.03$ & $-1.50 \pm 0.03$ & $-1.43 \pm 0.03$ & $-1.45 \pm 0.03$ \\
\hline NGC1291 & 230 & 41.36 & 39.33 & $-2.42 \pm 0.12$ & $-2.54 \pm 0.13$ & $-2.23 \pm 0.19$ & $-2.30 \pm 0.18$ \\
\hline NGC1313 & 236 & 41.43 & 39.12 & $-1.60 \pm 0.06$ & $-1.67 \pm 0.07$ & $-1.56 \pm 0.06$ & $-1.62 \pm 0.06$ \\
\hline NGC1487 & 76 & 41.92 & 39.14 & $-1.50 \pm 0.10$ & $-1.45 \pm 0.12$ & $-1.35 \pm 0.12$ & $-1.36 \pm 0.13$ \\
\hline NGC1512 & 314 & 41.81 & 39.24 & $-1.93 \pm 0.07$ & $-1.86 \pm 0.06$ & $-1.80 \pm 0.07$ & $-1.79 \pm 0.08$ \\
\hline NGC1744 & 218 & 40.76 & 38.74 & $-1.53 \pm 0.07$ & $-1.54 \pm 0.07$ & $-1.53 \pm 0.08$ & $-1.73 \pm 0.12$ \\
\hline NGC2366 & 191 & 41.61 & 38.46 & $-1.78 \pm 0.07$ & $-1.86 \pm 0.08$ & $-1.58 \pm 0.08$ & $-1.56 \pm 0.11$ \\
\hline NGC2403 & 1727 & 41.73 & 38.32 & $-1.64 \pm 0.02$ & $-1.71 \pm 0.03$ & $-1.56 \pm 0.03$ & $-1.63 \pm 0.03$ \\
\hline NGC2500 & 88 & 41.18 & 39.08 & $-1.41 \pm 0.10$ & $-1.39 \pm 0.12$ & $-1.38 \pm 0.10$ & $-1.38 \pm 0.12$ \\
\hline NGC2552 & 69 & 40.75 & 39.41 & $-2.02 \pm 0.22$ & $-1.96 \pm 0.19$ & $-1.59 \pm 0.28$ & $-1.85 \pm 0.19$ \\
\hline NGC2903 & 421 & 42.57 & 39.21 & $-1.59 \pm 0.04$ & $-1.65 \pm 0.04$ & $-1.47 \pm 0.05$ & $-1.53 \pm 0.05$ \\
\hline NGC2976 & 59 & 41.37 & 39.25 & $-1.65 \pm 0.16$ & $-1.85 \pm 0.12$ & $-1.48 \pm 0.14$ & $-1.59 \pm 0.12$ \\
\hline NGC3031 & 1559 & 41.36 & 38.56 & $-1.69 \pm 0.03$ & $-1.58 \pm 0.02$ & $-1.63 \pm 0.03$ & $-1.71 \pm 0.04$ \\
\hline NGC3239 & 106 & 41.96 & 39.77 & $-1.86 \pm 0.13$ & $-1.84 \pm 0.11$ & $-1.68 \pm 0.13$ & $-1.58 \pm 0.12$ \\
\hline NGC3274 & 58 & 41.00 & 38.63 & $-1.47 \pm 0.11$ & $-1.65 \pm 0.20$ & $-1.37 \pm 0.10$ & $-1.46 \pm 0.19$ \\
\hline NGC3344 & 474 & 41.63 & 39.06 & $-1.75 \pm 0.05$ & $-1.78 \pm 0.06$ & $-1.60 \pm 0.07$ & $-1.58 \pm 0.08$ \\
\hline NGC3351 & 351 & 42.48 & 39.30 & $-1.80 \pm 0.05$ & $-2.00 \pm 0.07$ & $-1.33 \pm 0.05$ & $-1.76 \pm 0.10$ \\
\hline NGC3368 & 159 & 41.47 & 39.61 & $-1.92 \pm 0.12$ & $-2.00 \pm 0.12$ & $-1.74 \pm 0.15$ & $-1.61 \pm 0.20$ \\
\hline NGC3486 & 283 & 41.36 & 39.38 & $-1.58 \pm 0.07$ & $-1.67 \pm 0.08$ & $-1.56 \pm 0.07$ & $-1.61 \pm 0.10$ \\
\hline NGC3521 & 285 & 41.35 & 38.88 & $-1.32 \pm 0.05$ & $-1.36 \pm 0.04$ & $-1.33 \pm 0.04$ & $-1.34 \pm 0.04$ \\
\hline NGC3627 & 125 & 42.24 & 39.03 & $-1.28 \pm 0.06$ & $-1.31 \pm 0.06$ & $-1.22 \pm 0.05$ & $-1.27 \pm 0.05$ \\
\hline NGC4068 & 41 & 40.39 & 38.79 & $-1.85 \pm 0.23$ & $-1.94 \pm 0.22$ & $-1.80 \pm 0.28$ & $-1.61 \pm 0.26$ \\
\hline NGC4214 & 669 & 41.46 & 38.00 & $-1.75 \pm 0.04$ & $-1.89 \pm 0.04$ & $-1.62 \pm 0.04$ & $-1.78 \pm 0.05$ \\
\hline NGC4236 & 862 & 41.01 & 38.62 & $-1.89 \pm 0.05$ & $-1.97 \pm 0.05$ & $-1.84 \pm 0.06$ & $-1.88 \pm 0.05$ \\
\hline NGC4242 & 95 & 40.57 & 39.38 & $-1.83 \pm 0.20$ & $-2.06 \pm 0.20$ & $-1.81 \pm 0.30$ & $-2.00 \pm 0.20$ \\
\hline NGC4258 & 767 & 42.09 & 38.82 & $-1.72 \pm 0.03$ & $-1.83 \pm 0.04$ & $-1.61 \pm 0.04$ & $-1.74 \pm 0.05$ \\
\hline NGC4288 & 56 & 41.04 & 38.59 & $-1.42 \pm 0.11$ & $-1.43 \pm 0.12$ & $-1.31 \pm 0.09$ & $-1.36 \pm 0.10$ \\
\hline NGC4395 & 949 & 41.22 & 38.41 & $-1.82 \pm 0.04$ & $-2.04 \pm 0.05$ & $-1.60 \pm 0.04$ & $-1.91 \pm 0.06$ \\
\hline NGC4449 & 239 & 41.85 & 39.00 & $-1.37 \pm 0.06$ & $-1.37 \pm 0.05$ & $-1.32 \pm 0.06$ & $-1.34 \pm 0.05$ \\
\hline NGC4490 & 160 & 41.74 & 39.60 & $-1.43 \pm 0.09$ & $-1.48 \pm 0.08$ & $-1.30 \pm 0.10$ & $-1.27 \pm 0.09$ \\
\hline NGC4618 & 86 & 41.24 & 40.16 & $-1.48 \pm 0.22$ & $-1.18 \pm 0.09$ & $-1.65 \pm 0.28$ & $-1.19 \pm 0.11$ \\
\hline NGC4625 & 92 & 41.19 & 38.49 & $-1.42 \pm 0.07$ & $-1.45 \pm 0.08$ & $-1.35 \pm 0.08$ & $-1.43 \pm 0.11$ \\
\hline NGC4707 & 41 & 40.15 & 39.04 & $-1.80 \pm 0.33$ & $-1.82 \pm 0.34$ & $-1.86 \pm 0.26$ & $-1.87 \pm 0.54$ \\
\hline NGC4736 & 379 & 41.68 & 38.12 & $-1.46 \pm 0.04$ & $-1.46 \pm 0.03$ & $-1.40 \pm 0.04$ & $-1.40 \pm 0.04$ \\
\hline NGC4826 & 57 & 41.92 & 39.97 & $-2.20 \pm 0.23$ & $-2.05 \pm 0.18$ & $-1.55 \pm 0.15$ & $-1.61 \pm 0.16$ \\
\hline NGC5055 & 661 & 41.27 & 38.95 & $-1.48 \pm 0.04$ & $-1.55 \pm 0.04$ & $-1.44 \pm 0.04$ & $-1.52 \pm 0.05$ \\
\hline NGC5068 & 221 & 41.39 & 39.61 & $-1.54 \pm 0.09$ & $-1.41 \pm 0.06$ & $-1.55 \pm 0.11$ & $-1.58 \pm 0.10$ \\
\hline NGC5194 & 488 & 41.92 & 39.38 & $-1.42 \pm 0.04$ & $-1.42 \pm 0.04$ & $-1.41 \pm 0.04$ & $-1.38 \pm 0.04$ \\
\hline NGC5195 & 33 & 41.20 & 38.81 & $-1.73 \pm 0.16$ & $-1.85 \pm 0.20$ & $-1.37 \pm 0.14$ & $-1.39 \pm 0.15$ \\
\hline NGC5204 & 173 & 40.59 & 38.95 & $-1.55 \pm 0.14$ & $-1.61 \pm 0.09$ & $-1.49 \pm 0.13$ & $-1.53 \pm 0.09$ \\
\hline NGC5236 & 856 & 43.05 & 38.63 & $-1.50 \pm 0.02$ & $-1.57 \pm 0.03$ & $-1.40 \pm 0.02$ & $-1.44 \pm 0.03$ \\
\hline NGC5457 & 2624 & 42.29 & 39.05 & $-1.66 \pm 0.02$ & $-1.68 \pm 0.02$ & $-1.54 \pm 0.02$ & $-1.61 \pm 0.02$ \\
\hline NGC5474 & 307 & 40.77 & 39.01 & $-1.77 \pm 0.09$ & $-1.79 \pm 0.08$ & $-1.76 \pm 0.09$ & $-1.81 \pm 0.09$ \\
\hline NGC5585 & 216 & 40.56 & 38.56 & $-1.56 \pm 0.08$ & $-1.62 \pm 0.08$ & $-1.69 \pm 0.11$ & $-1.64 \pm 0.09$ \\
\hline NGC5832 & 74 & 40.30 & 39.01 & $-1.46 \pm 0.16$ & $-1.46 \pm 0.17$ & $-1.47 \pm 0.17$ & $-1.30 \pm 0.17$ \\
\hline NGC7793 & 731 & 40.83 & 38.27 & $-1.33 \pm 0.03$ & $-1.44 \pm 0.04$ & $-1.30 \pm 0.03$ & $-1.40 \pm 0.04$ \\
\hline SextansA & 279 & 38.95 & 37.57 & $-1.80 \pm 0.11$ & $-1.86 \pm 0.12$ & $-1.77 \pm 0.11$ & $-1.61 \pm 0.09$ \\
\hline UGC00668 & 1813 & 38.80 & 36.69 & $-1.99 \pm 0.04$ & $-2.16 \pm 0.05$ & $-1.92 \pm 0.04$ & $-2.07 \pm 0.05$ \\
\hline UGC01176 & 45 & 40.18 & 39.11 & $-2.02 \pm 0.32$ & $-1.69 \pm 0.31$ & $-1.81 \pm 0.25$ & $-1.61 \pm 0.23$ \\
\hline UGC01249 & 88 & 40.86 & 39.86 & $-2.16 \pm 0.39$ & $-2.12 \pm 0.25$ & $-2.08 \pm 0.28$ & $-2.03 \pm 0.36$ \\
\hline UGC04305 & 422 & 40.77 & 37.95 & $-1.76 \pm 0.05$ & $-1.76 \pm 0.05$ & $-1.70 \pm 0.05$ & $-1.68 \pm 0.06$ \\
\hline UGC05139 & 85 & 39.65 & 38.31 & $-1.78 \pm 0.21$ & $-2.35 \pm 0.29$ & $-1.83 \pm 0.23$ & $-1.76 \pm 0.27$ \\
\hline UGC05336 & 49 & 39.07 & 38.31 & $-2.16 \pm 0.41$ & $-2.07 \pm 1.19$ & $-2.31 \pm 0.46$ & $-2.09 \pm 1.32$ \\
\hline UGC05340 & 35 & 39.89 & 38.64 & $-1.31 \pm 0.23$ & $-1.21 \pm 0.20$ & $-1.10 \pm 0.18$ & $-1.06 \pm 0.25$ \\
\hline
\end{tabular}


Table 2 - continued

\begin{tabular}{|c|c|c|c|c|c|c|c|}
\hline $\begin{array}{l}\text { Galaxy } \\
\text { Name } \\
\text { (1) }\end{array}$ & $\begin{array}{c}\mathrm{N}_{\text {srcs }} \\
\text { (No.) } \\
(2)\end{array}$ & $\begin{array}{c}\log \left(\mathrm{L}_{\max }\right) \\
\left(\mathrm{erg} \mathrm{s}^{-1}\right) \\
(3)\end{array}$ & $\begin{array}{c}\log (\text { Lum Peak }) \\
\left(\mathrm{erg} \mathrm{s}^{-1}\right) \\
(4)\end{array}$ & $\begin{array}{c}\alpha \\
\text { EqNum-CI } \\
(5)\end{array}$ & $\begin{array}{c}\alpha \\
\text { EqNum-Avg } \\
(6)\end{array}$ & $\begin{array}{c}\alpha \\
\text { EqSize-CI } \\
(7)\end{array}$ & $\begin{array}{c}\alpha \\
\text { EqSize-Avg } \\
(8)\end{array}$ \\
\hline UGC05364 & 107 & 37.90 & 36.83 & $-2.10 \pm 0.23$ & $-1.78 \pm 0.24$ & $-1.88 \pm 0.22$ & $-1.70 \pm 0.27$ \\
\hline UGC05373 & 130 & 39.01 & 37.73 & $-2.20 \pm 0.20$ & $-2.28 \pm 0.23$ & $-2.06 \pm 0.17$ & $-2.16 \pm 0.27$ \\
\hline UGC05666 & 564 & 40.77 & 38.20 & $-1.65 \pm 0.04$ & $-1.86 \pm 0.06$ & $-1.60 \pm 0.05$ & $-1.79 \pm 0.06$ \\
\hline UGC05829 & 133 & 40.65 & 39.03 & $-1.80 \pm 0.12$ & $-1.78 \pm 0.13$ & $-1.63 \pm 0.12$ & $-1.72 \pm 0.13$ \\
\hline UGC05889 & 35 & 40.02 & 38.91 & $-2.22 \pm 0.38$ & $-2.47 \pm 0.53$ & $-1.99 \pm 0.50$ & $-1.87 \pm 0.63$ \\
\hline UGC06817 & 75 & 39.43 & 37.88 & $-2.22 \pm 0.19$ & $-2.18 \pm 0.25$ & $-1.64 \pm 0.23$ & $-1.82 \pm 0.28$ \\
\hline UGC07559 & 52 & 39.78 & 38.37 & $-1.62 \pm 0.19$ & $-1.60 \pm 0.16$ & $-1.58 \pm 0.15$ & $-1.50 \pm 0.15$ \\
\hline UGC07577 & 62 & 39.22 & 38.16 & $-1.97 \pm 0.31$ & $-2.10 \pm 0.30$ & $-2.48 \pm 0.47$ & $-2.15 \pm 0.36$ \\
\hline UGC07608 & 66 & 40.51 & 39.23 & $-1.83 \pm 0.24$ & $-1.89 \pm 0.19$ & $-1.63 \pm 0.24$ & $-1.58 \pm 0.19$ \\
\hline UGC07690 & 30 & 40.95 & 39.44 & $-1.79 \pm 0.27$ & $-1.80 \pm 0.26$ & $-1.73 \pm 0.26$ & $-1.75 \pm 0.26$ \\
\hline UGC07698 & 124 & 39.93 & 38.34 & $-1.91 \pm 0.12$ & $-1.76 \pm 0.10$ & $-1.72 \pm 0.12$ & $-1.80 \pm 0.18$ \\
\hline UGC07866 & 76 & 40.09 & 38.54 & $-2.00 \pm 0.17$ & $-2.07 \pm 0.16$ & $-1.78 \pm 0.19$ & $-1.87 \pm 0.22$ \\
\hline UGC07949 & 38 & 40.15 & 39.01 & $-2.06 \pm 0.28$ & $-3.23 \pm 0.56$ & $-2.46 \pm 0.46$ & $-2.84 \pm 0.58$ \\
\hline UGC08024 & 59 & 39.27 & 38.45 & $-1.41 \pm 0.29$ & $-1.99 \pm 0.36$ & $-1.30 \pm 0.26$ & $-1.61 \pm 0.33$ \\
\hline UGC08188 & 176 & 39.97 & 38.95 & $-2.46 \pm 0.28$ & $-2.11 \pm 0.18$ & $-2.12 \pm 0.24$ & $-2.00 \pm 0.18$ \\
\hline UGC08201 & 67 & 39.49 & 38.50 & $-2.23 \pm 0.33$ & $-2.69 \pm 0.33$ & $-2.17 \pm 0.30$ & $-1.85 \pm 0.27$ \\
\hline UGC08651 & 33 & 39.52 & 38.19 & $-2.54 \pm 0.34$ & $-2.55 \pm 0.42$ & $-2.22 \pm 0.39$ & $-1.95 \pm 0.41$ \\
\hline UGC12613 & 33 & 38.38 & 37.10 & $-2.60 \pm 0.41$ & $-2.71 \pm 0.37$ & $-1.85 \pm 0.30$ & $-2.40 \pm 0.39$ \\
\hline UGCA106 & 135 & 40.90 & 39.45 & $-1.95 \pm 0.16$ & $-2.07 \pm 0.16$ & $-1.97 \pm 0.19$ & $-1.87 \pm 0.17$ \\
\hline WLM & 407 & 39.04 & 37.23 & $-1.95 \pm 0.08$ & $-2.06 \pm 0.09$ & $-1.85 \pm 0.09$ & $-1.99 \pm 0.11$ \\
\hline
\end{tabular}

Notes. The properties of the sources identified in each galaxy and the luminosity functions constructed from these sources. Column 1: Galaxy name. Column 2: the number of sources identified in each galaxy. Column 3: the peak of the luminosity histogram for sources in each galaxy. Column 4: the luminosity of the brightest source found in each galaxy. Columns 5-8: the LF slopes $(\alpha)$ measured using four combinations of two binning methods and two aperture correction methods: (1) EqNum-CI: CI-based aperture correction and equal number binning; (2) EqNum-Avg: average aperture correction and equal number binning; (3) Eq Size-CI: CI-based aperture correction and equal luminosity size binning; (4) EqSize-Avg: average aperture correction and equal luminosity size binning.

the dust reddening curve of Draine (2003). Lee et al. (2009) quotes a 20 per cent scatter for galaxies with $-14.7>M_{B}>-18$ and only 10 per cent for galaxies fainter than -14.7 . Since the majority $(N=$ $36)$ of these 47 galaxies are low-luminosity dwarfs $\left(M_{B}>-14.7\right)$ and the remaining galaxies are fainter than $M_{B}>-18$, we do not expect these galaxies to add a significant amount of scatter to the trends presented in Section 7.

\section{2 $M_{\mathrm{B}, 0}$}

The apparent optical fluxes are taken from Cook et al. (2014a) measured inside the UV apertures of Lee et al. (2011), where the optical images have been cleaned of contaminating sources (i.e. background galaxies and foreground stars). These optical fluxes have been converted to absolute magnitudes via the distance moduli of Kennicutt et al. (2008), and corrected for internal dust correction (see previous section).

\subsection{Stellar mass}

We utilize the LVL stellar masses of Cook et al. (2014b) which are derived from a constant Spitzer $3.6 \mu \mathrm{m}$ mass-to-light ratio $\left(\Upsilon_{\star}^{3.6 \mu \mathrm{m}}\right)$ of 0.5 . In recent years, a consistent $\Upsilon_{\star}^{3.6 \mu \mathrm{m}}$ of 0.5 has begun to emerge between population synthesis models and the baryonic Tully-Fisher relation measurements (Oh et al. 2008; Eskew, Zaritsky \& Meidt 2012; Barnes et al. 2014; McGaugh \& Schombert 2014; Meidt et al. 2014; McGaugh \& Schombert 2015).

\subsection{SFR, $\Sigma_{\mathrm{SFR}}, \mathrm{SSFR}$}

The SFRs are derived via FUV fluxes taken from the work of Lee et al. (2011) where we corrected for internal extinction as discussed in Section 6.1. We use the prescription of Murphy et al. (2011) to convert the dust-corrected FUV fluxes into a SFR where the uncertainties on the SFR include only the photometric uncertainties. The SFR prescriptions of Murphy et al. (2011) are an updated version of Kennicutt (1998) using a Kroupa (Kroupa 2001) IMF. The calibration coefficient of Kennicutt (1998) is a factor of $\sim 1.6$ larger than the Murphy et al. (2011) coefficient [see Calzetti et al. (2007)].

The $\Sigma_{\text {SFR }}$ values are derived via normalizing the SFR by the area of the galaxy aperture. We choose to define our areas by the R25 optical apertures instead of the UV apertures since the R25 apertures are more self-consistent as these apertures are defined as the isophotal ellipse at which the optical surface brightness equals 25 mag $\operatorname{arcsec}^{-2}$ in the $B$-band. For example, there are many galaxies whose UV aperture extends well beyond the extent of our FUV identified sources, thus leaving a non-negligible fraction of the area as empty space. This occurs for the following reasons: (1) irregular morphology of dwarfs and non-symmetric spiral features in spirals can result in UV apertures which are skewed in one direction relative to the centre of the galaxy leaving empty space inside the UV aperture on the opposite side (e.g. NGC2366, NGC5457/M101); and (2) many galaxies have extended, low-surface brightness UV emission at large radii (i.e. XUV galaxies; Thilker et al. 2007) with a few, or often no, identifiable FUV sources above a $3 \sigma$ detection threshold. However, we verify that using either the UV or IR apertures do not significantly affect our results given in Section 7. The 


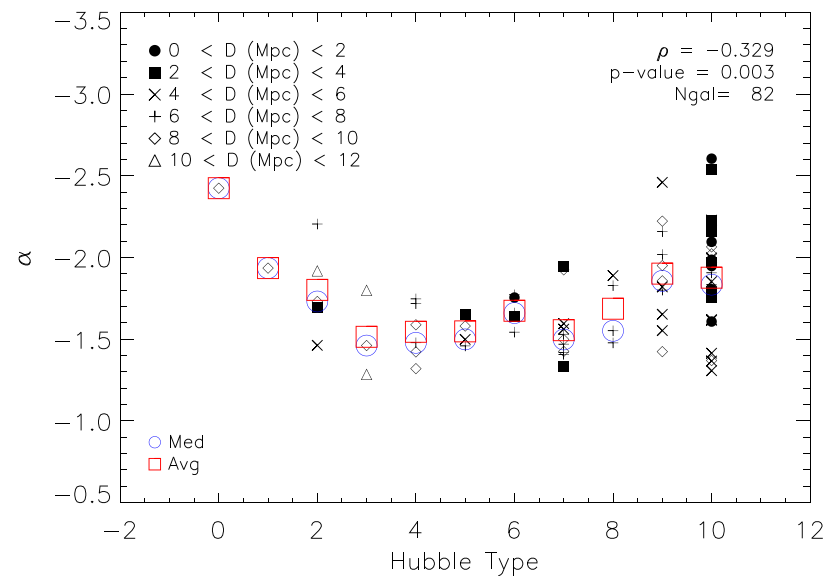

Figure 10. A plot of the LF slope $(\alpha)$ versus optical morphology. The symbols are defined in the lower-left corner, where we have binned our galaxies according to their distance. Filled symbols represent closer galaxies and open symbols represent distant galaxies. The number of galaxies and the Spearman correlation coefficient $(\rho)$ are indicated in the upper right legend. We find no clear trend between $\alpha$ and morphology, and find increased scatter for later-type galaxies $(T \geq 9)$.

specific SFRs (sSFRs) are derived by normalizing the SFRs by the LVL stellar mass discussed in the previous section.

\subsection{Metallicity}

We utilize the metallicity catalogue compiled by Cook et al. (2014b). This catalogue is an updated version of the Marble et al. (2010) compilation, where both with 'direct' and 'strong-line' oxygen abundances measured by Berg et al. (2012) and 'strong-line' oxygen abundances from Moustakas et al. (2010) were added. Cook et al. (2014b) replaced all previous 'strong-line' abundances with 'direct' abundances and updated any previous 'strong-line' abundances with newer 'strong-line' abundances. The majority of the 'strong-line' metallicities are on similar calibration scales (i.e. similar to McGaugh 1991). The final metallicity sample is composed of roughly half 'direct' and half 'strong-line' values.

\section{RESULTS: TRENDS WITH GALAXY PROPERTIES}

In this section, we examine the relationships between LF slope and global galaxy properties, where LF slope forms the strongest trends with SFR properties. We end this section with an evaluation of how our choice of dust correction, aperture correction, and LF binning affect the strength of the relationships between LF slope and galaxy properties. We find that the trends found between LF slope and galaxy environment are not significantly affected by these choices.

\subsection{LF trends}

Fig. 10 is a plot of the LF slope $(\alpha)$ versus optical morphology for the LVL sample. The symbols for individual galaxies are described in the upper-left legend representing distance bins in intervals of $2 \mathrm{Mpc}$, where filled symbols represent closer galaxies and open symbols represent distant galaxies. The larger open squares and open circles represent the average and median, respectively, of the galaxies in each morphological type.

We find no discernible trend with galaxy type in Fig. 10. This is supported by the small Spearman correlation coefficient $(\rho)$ of

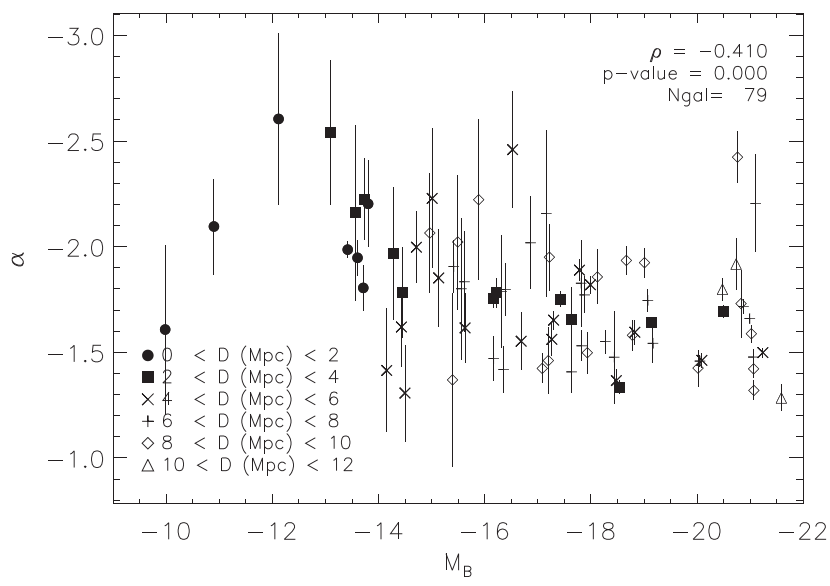

Figure 11. A plot of the LF slope $(\alpha)$ versus absolute $B$-band magnitude $\left(M_{B}\right)$ similar to that of Fig. 10. We find a weak trend between these two quantities.

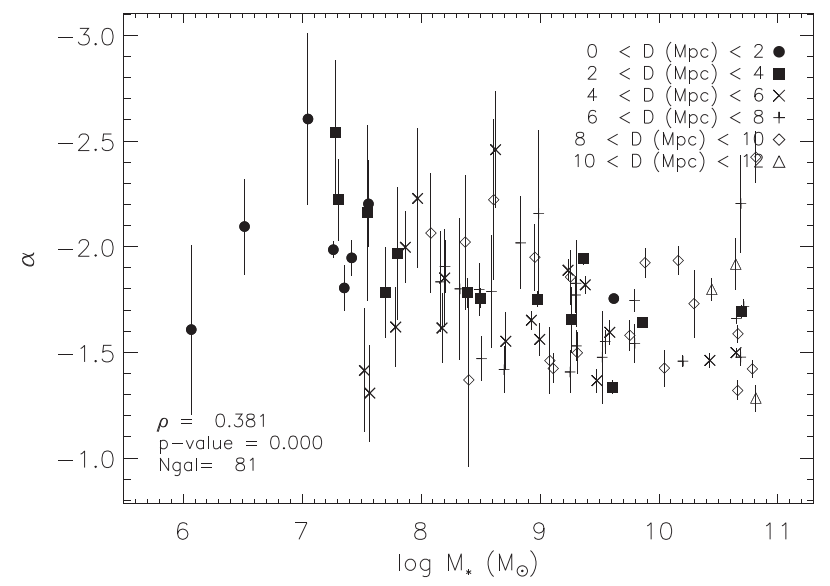

Figure 12. A plot of the LF slope $(\alpha)$ versus stellar mass $\left(M_{\star}\right)$ similar to that of Fig. 10. We find a weak trend between these two quantities similar to that found for $M_{B}$.

-0.329 . We also find that there is increased scatter for later galaxy types $(T \geq 9)$, which are generally the lower-mass, dwarf galaxies. It should also be noted that the majority of the dwarf galaxies tend to be closer (i.e. filled symbols) and have fewer sources.

Fig. 11 is a plot of $\alpha$ versus the absolute $B$-band magnitude $\left(M_{B}\right)$, which has been corrected for internal dust extinction. We find the LF slope shows a weak correlation with $M_{B}$, where low-luminosity galaxies tend to have steeper (i.e. more negative) slopes. The weak trend is supported by a correlation coefficient of -0.410 .

Fig. 12 is a plot $\alpha$ versus the stellar mass $\left(M_{\star}\right)$. We find the LF slope shows a weak correlation with $M_{\star}$, where low-mass galaxies tend to have steeper slopes. The trend is supported by a correlation coefficient of 0.381 . We note that this trend is similar to that of galaxy luminosity $\left(M_{B}\right)$ due to the tight correlation between optical luminosity and $M_{\star}$ (Cook et al. 2014b).

Fig. 13 is a plot of $\alpha$ versus the metallicity $(12+\log (\mathrm{O} / \mathrm{H}))$ for 82 of the LVL galaxies. The metallicities were taken from the most recent compilation of the LVL galaxies by Cook et al. (2014b). We find no trend with a correlation coefficient of $\rho \sim 0.156$. The lack of a trend is somewhat surprising since metallicity forms correlations with both $M_{B}$ and $M_{\star}$ (e.g. Lee, Grebel \& Hodge 2003; Lee, Zucker \& Grebel 2007; Berg et al. 2012). 


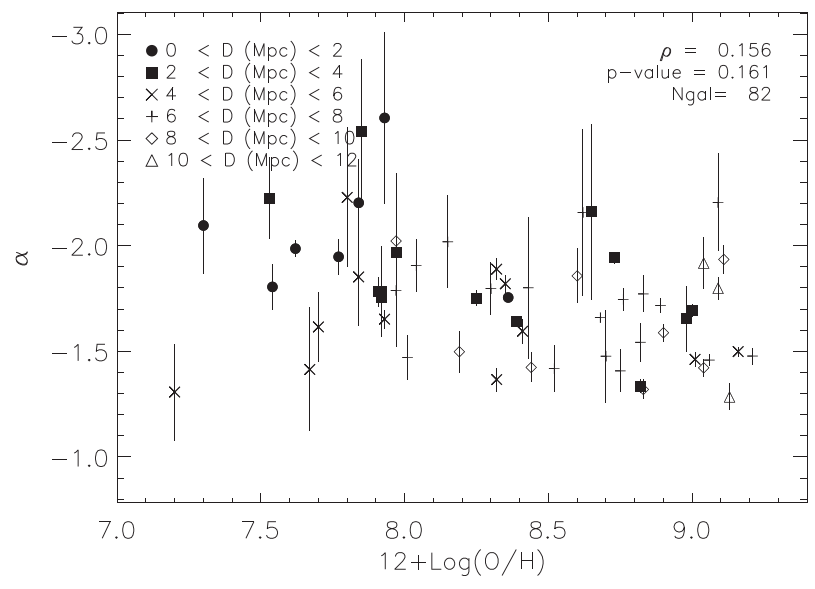

Figure 13. A plot of the LF slope $(\alpha)$ versus metallicity similar to that of Fig. 10. We find a no trend between these two quantities.

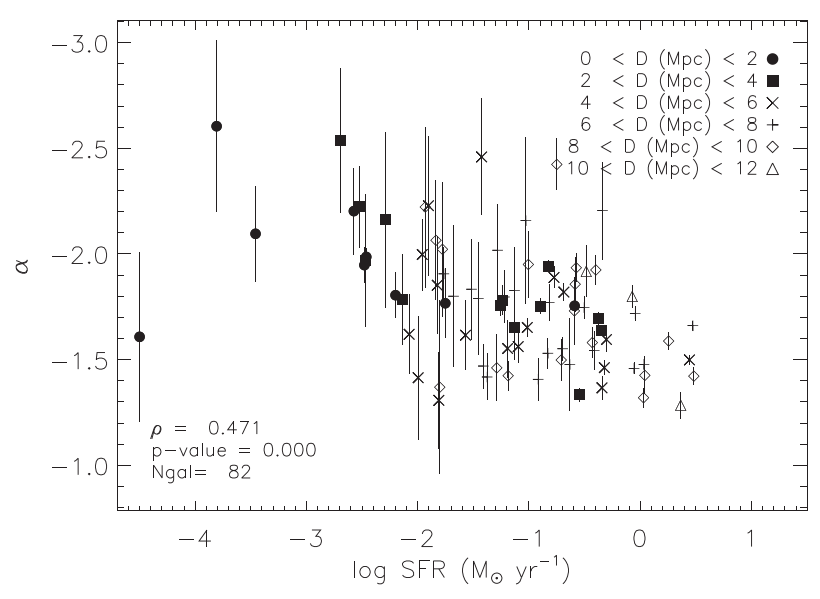

Figure 14. A plot of the LF slope $(\alpha)$ versus SFR similar to that of Fig. 10. We find a weak-to-moderately strong trend between these two quantities.

Fig. 14 is a plot of $\alpha$ versus the FUV-derived SFR which has been corrected for internal dust extinction. We find a weak-to-moderate correlation ( $\rho=0.471$ ) between $\alpha$ and SFR, where low-SFR galaxies tend to have steeper slopes. Despite the tight correlations between SFR and $M_{\star}$ and $M_{B}$ (e.g. Ellison et al. 2008; Salim et al. 2014), the stronger trend between $\alpha$ and SFR suggests that the properties of star-forming regions are more closely connected to the star formation properties of a galaxy.

Fig. 15 is a plot of $\alpha$ versus $\mathrm{SSFR}$ (sSFR $\equiv \mathrm{SFR} / \mathrm{M}_{\star}$ ), which is the global SFR of a galaxy normalized by the total stellar mass. Despite the weak-to-moderate relationship between $\alpha$ and SFR, we find no trend between LF slope and $\operatorname{sSFR}(\rho=0.096)$.

Fig. 16 is a plot of $\alpha$ versus SFR density $\left(\Sigma_{\mathrm{SFR}} \equiv \mathrm{SFR} / \mathrm{kpc}^{2}\right)$, which is the global SFR of a galaxy normalized by the area of the galaxy. We find that $\Sigma_{\text {SFR }}$ exhibits a moderately strong correlation ( $\rho=0.639$; the strongest for any global galaxy property) with LF slope, where low $\Sigma_{\mathrm{SFR}}$ environments tend to have steeper slopes. It is interesting to note that the strength of the trends between two global star formation properties (sSFR and $\Sigma_{\mathrm{SFR}}$ ) is drastically different from the trend with SFR when normalizing by $M_{\star}$ and area. The moderate trend between $\alpha$ and $\Sigma_{\mathrm{SFR}}$ and no trend between $\alpha$ and sSFR suggests that the density of star formation is physically connected to the properties of star-forming regions.

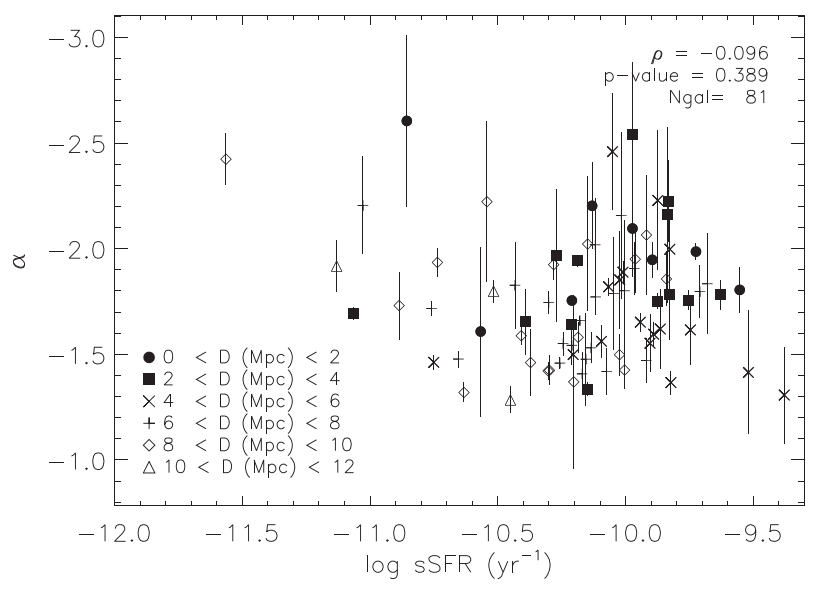

Figure 15. A plot of the LF slope $(\alpha)$ versus sSFR similar to that of Fig. 10. We find no trend between these two quantities.

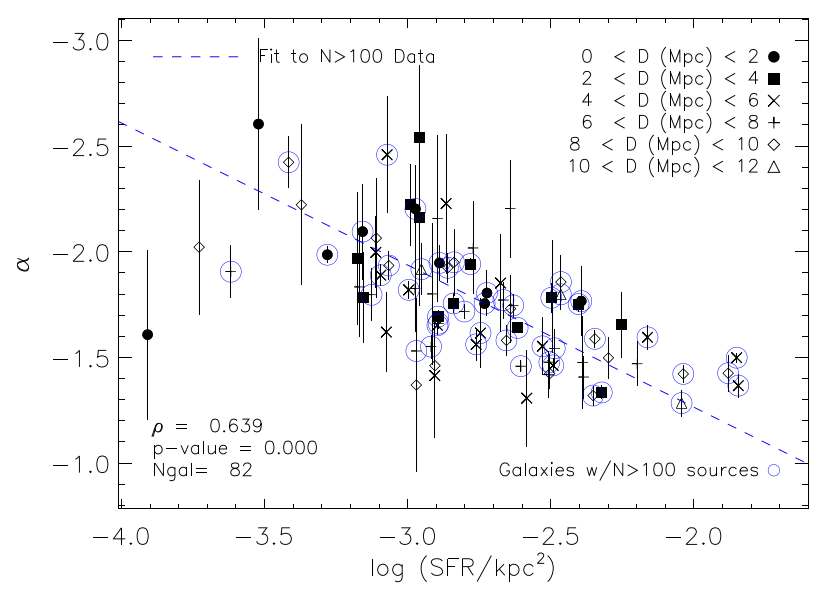

Figure 16. A plot of the LF slope $(\alpha)$ versus $\Sigma_{\mathrm{SFR}}$ similar to that of Fig. 10. We find a moderately strong trend between these two quantities. The blue-open circles represent galaxies with a total number of sources greater than 100 where we expect random scatter due to stochastic effects to be minimal. The correlation coefficient for galaxies with $N>100$ is 0.705 ; stronger than that of all galaxies in the figure. The blue-dashed line is a bisector fit to the $N>100$ data and is described by equation (7).

In Fig. 16, we have also overlaid large blue circles on to the symbols of galaxies with a total number of sources greater than 100 , where it is possible that low-number statistics may add random scatter to LF slopes (see Section 8.1.1). We find that correlation between LF slope and $\Sigma_{\text {SFR }}$ is reproduced when using only galaxies with good number statistics, and that these galaxies span nearly the full range of the entire galaxy sample. Furthermore, we find that the correlation coefficient is stronger for $N>100$ data with a value of 0.705 . To quantify the relationship between LF slope and $\Sigma_{\mathrm{SFR}}$, we perform a bisector fit to the $N>100$ data which is described by the equation:

$\alpha=0.67 \times \log \Sigma_{\mathrm{SFR}}+0.08$.

\subsection{The effects of dust correction, aperture correction, and LF binning}

In previous sections, we have made choices on how to obtain the total, unobscured luminosities of our regions and how to bin these luminosities to construct our LFs and consequently measure the LF 


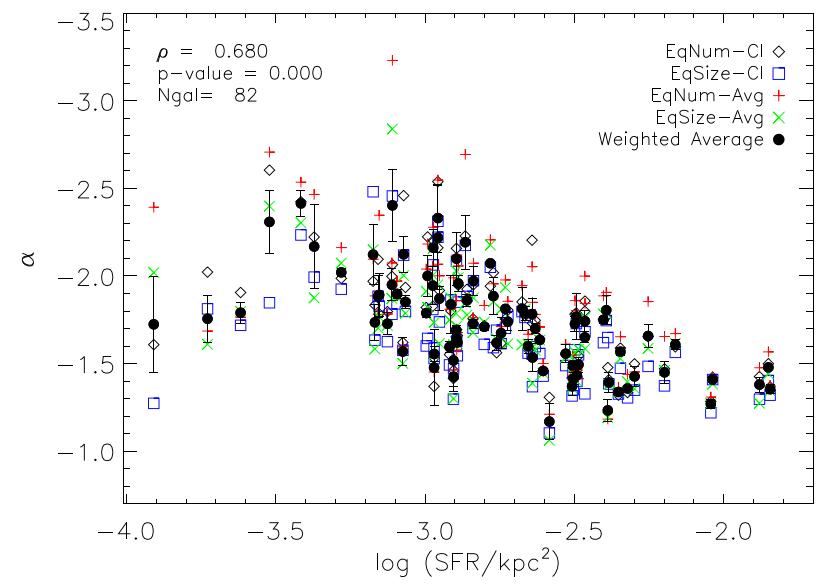

Figure 17. A plot of the LF slope $(\alpha)$ versus $\Sigma_{\text {SFR }}$ where we have combined two photometry methods and two LF binning methods to produce four combinations of the LF slope. This graphically shows the systematics of our methods. We find a strong trend between these $\alpha$ and $\Sigma_{\mathrm{SFR}}$ when using a weighted average LF slope for the four combinations of photometry and binning methods.

slopes. In our analysis we have chosen a fiducial set of methods (deriving our own dust correction for individual regions, CI-based aperture correction, equal numbering binning) which represent what we believe to be a reasonable set of methods that minimizes any biases. In this section, we provide an evaluation on our choice of dust correction, aperture correction, and LF binning on the strength of the trends between LF slope and galaxy environment.

To test the effects of our dust correction for individual regions, we evaluate the strength of the trend between LF slope and $\Sigma_{\text {SFR }}$ (i.e. the strongest trend) when applying no dust correction, the galaxy-wide correction of Hao et al. (2011), and previous corrections derived for individual regions (Leroy et al. 2008; Liu et al. 2011). We find that the plots for no correction, galaxy-wide correction, and the correction derived for regions are very similar in shape and structure to Fig. 16, and that the correlation coefficients are $0.402,0.523$, and 0.561 , respectively. The persistence of a trend between $\alpha$ and $\Sigma_{\text {SFR }}$ when using no dust correction and two different dust corrections suggests that our results are not significantly sensitive to the choice of dust correction methods.

The effects of aperture correction and LF binning can be evaluated via generating four sets LF slopes by combining the two choices of aperture corrections and the two LF binning methods for each galaxy: (1) EqNum-CI: CI-based aperture correction and equal number binning; (2) EqNum-Avg: average aperture correction and equal number binning; (3) EqSize-CI: CI-based aperture correction and equal luminosity size binning and (4) EqSize-Avg: average aperture correction and equal luminosity size binning.

Fig. 17 illustrates the variability of our trends when using different methods where the LF slopes of all four combinations are plotted against $\Sigma_{\mathrm{SFR}}$ (i.e. the strongest trend). The symbols with different colours represent the results for each combination of aperture correction and LF binning, and the filled circles represent the weighted average for the four combinations of methods. There are a handful of galaxies where the LF slope for one or two methods are outliers from the weighted average (i.e. $\Sigma_{\mathrm{SFR}} \sim-3.9$ with $-1.3<$ $\Delta \alpha<-2.4$ ); however, the majority of galaxies show only small LF slope differences between the four combinations of methods.

It is interesting to note that the weighted average of all four LF slopes forms a stronger correlation with $\Sigma_{\mathrm{SFR}}$ compared to any

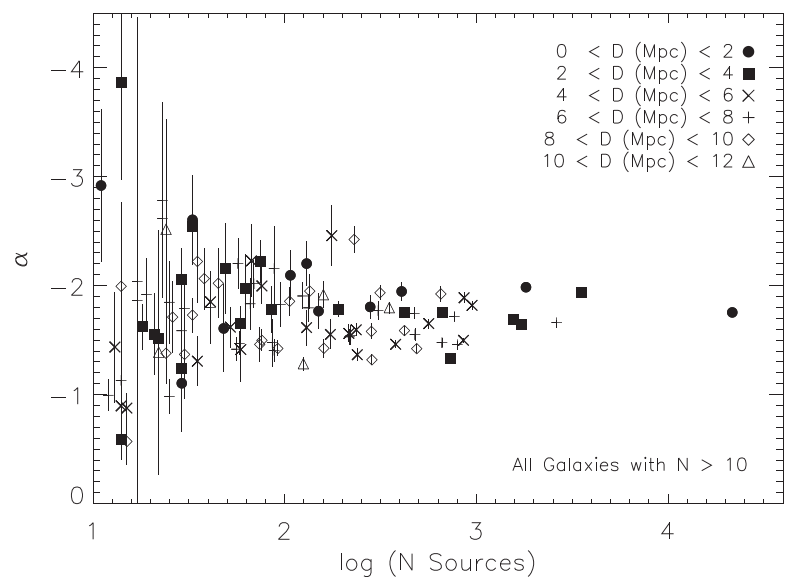

Figure 18. A plot of the LF slope $(\alpha)$ versus number of sources similar to that of Fig. 10. We find increased scatter towards lower numbers of sources, and decreased scatter towards higher numbers of sources. The average slope of all LFs is $-1.76 \pm 0.30$ (median $=-1.75$ ).

single method. This moderate-to-strong correlation $(\rho \sim 0.680)$ between the weighted average $\alpha$ values and $\Sigma_{\mathrm{SFR}}$ suggests that our results are robust to our choice of photometry and luminosity binning.

\section{DISCUSSION}

In this section, we investigate observational effects that may lead to artificial trends in the LF slope. We ultimately find that these effects cannot fully account for the correlations between LF slope and SFR nor $\Sigma_{\text {SFR }}$. We then compare our LF slope-host galaxy trends to previous studies, and end the section with a discussion of the implications of these trends on the star formation process.

\subsection{Competing effects}

In this section, we provide a careful examination of two competing factors that can change the LF slope: stochastic sampling of the LF in galaxies with low-number statistics and blending of sources at lower resolution due to distance. To quantify these effects, we simulate the scatter in LF slope expected given the number of sources in each galaxy, and degrade the resolution of two large, nearby spiral galaxies to greater distances and re-measure the LF slopes.

\subsubsection{Stochastic sampling of the LF}

The luminosity distributions of star-forming regions follow a power law, where there are fewer bright regions than faint regions. Sampling a luminosity distribution with low-number statistics over many iterations results in the random presence of bright regions that can affect the slope of the distribution. For example, in a distribution where there is expected to be just one region in the brightest luminosity bin, the presence of many bright regions with low probabilities would flatten the LF slope while the presence of no bright regions would steepen the LF slope.

It is not clear a priori if stochastic scatter in $\alpha$ introduces a bias (i.e. if the LF slope is systematically steeper or flatter for galaxies with low-number statistics) nor is the amplitude of this scatter constrained. A first-order check of both the potential systematic bias and quantification of this scatter can be seen in Fig. 18, where $\alpha$ is plotted against the total number of sources in each galaxy. Visual inspection of Fig. 18 reveals that $\alpha$ shows increased scatter for 
galaxies with lower numbers of star-forming regions $(N \lesssim 100)$, and that $\alpha$ converges to a value slightly flatter than -2 . The median of all good LFs is $-1.75 \pm 0.30$ (the average is -1.76 ).

The random scatter of $\alpha$ values at lower number statistics suggests that stochasticity does not systematically bias the LF slope to either steeper nor flatter slopes. Also, it is not clear why our LFs converge to flatter slopes than those found in star cluster and $\mathrm{H} \alpha$ studies of local spiral and dwarf galaxies; -2 . However, a recent star cluster study using multiband $(N U V, U, B, V, R) H S T$ imaging has found a systematic trend for LF slopes derived from the same star clusters at different wavelengths, where the LF slopes derived from shorter wavelengths tend to have flatter slopes (Adamo et al., in preparation). It is plausible that the flatter FUV-derived LF slopes measured here are an extension of this trend with shorter wavelengths.

We can also make a first-order check on the effects that lownumber statistics might have on the trends found in Section 7 between LF slope and galaxy properties via examination of these trends when only using galaxies with a total number of sources greater 100. The blue circles of Fig. 16 represent galaxies with $N$ $>100$ and form a stronger correlation between LF slope and $\Sigma_{\text {SFR }}$. This stronger relationship is supported by the Spearman correlation coefficient of $\rho=0.705$ for $N>100$ galaxies. In addition, the properties of these galaxies span nearly the full range of both LF slope and $\Sigma_{\text {SFR }}$ as the entire galaxy sample. The persistence of the trend between LF slope and $\Sigma_{\mathrm{SFR}}$ for galaxies with good number statistics suggests that stochasticity is not responsible for this trend.

To quantify this scatter, we simulate the expected change in $\alpha$ given the number of sources in each galaxy. For our simulations, we assume a universal LF that can be described by a power law with a slope of -2 . For each galaxy, we randomly draw star-forming regions equal to the number of sources found in the galaxy and limit these sources to luminosities above the peak in the real luminosity histogram and below the luminosity of the brightest star-forming region found in the entire sample. The lower luminosity limit mimics the sensitivity of the FUV image, and the upper limit allows for the possibility of creating random bright objects. The subsequent LF is then fit with the same methods used to fit our real LFs and we iterate 1000 times.

Fig. 19 shows the simulated $\alpha$ values plotted against the total number of sources found in each galaxy, where the red diamonds represent the median of all simulations, the median error bars represent the 68 th and 32 nd percentile of the simulations (i.e. the $1 \sigma$ confidence interval), and the grey dots represent all simulated realizations of the LF slope. For comparison purposes, we have overplotted the real data points measured for each galaxy represented by filled-blue circles.

Visual inspection of Fig. 19 shows that the median of all simulations randomly scatter around a -2 slope for galaxies with both high- and low-number statistics, but find that the scatter in the simulations increases symmetrically around a slope of -2 towards galaxies with low-number statistics. When comparing the real LF slopes to the simulated slopes, we find that galaxies with highnumber statistics $(N>100)$ tend to have flatter slopes than the simulations. However, for galaxies with low-number statistics, we find similar distributions of scatter between our simulations and real data, where the scatter is not systematic, but rather randomly distributed both above and below the input -2 LF slope. The random scatter seen in both the observed and simulated LF slopes in galaxies with low-number statistics (i.e. where we expect stochasticity to be prominent) suggests that stochastic scatter is not responsible for the trends found between LF slope and galaxy properties.

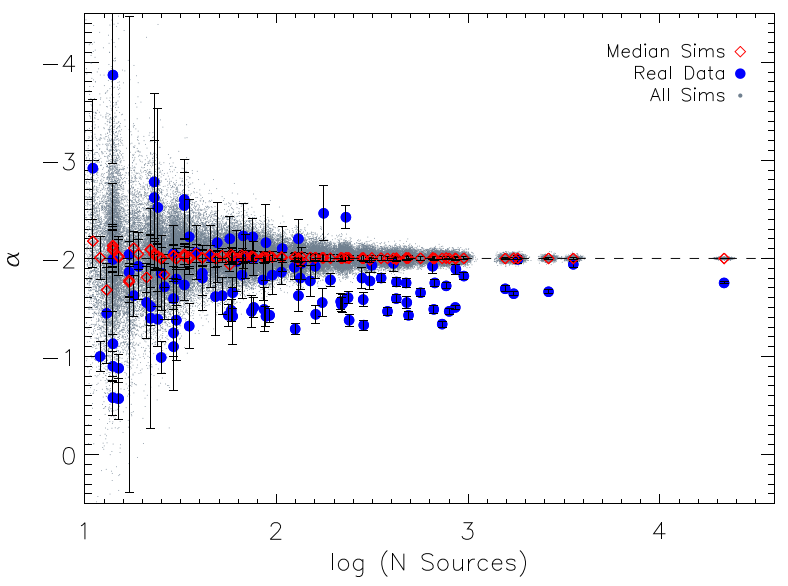

Figure 19. A plot of simulated LF slope $(\alpha)$ versus the number of sources, where the red diamonds represent the median of all simulations, the median error bars represent the 68th and 32nd percentile of the simulations (i.e. the $1 \sigma$ confidence interval), and the grey dots represent all simulated realizations of the LF slope. In addition, the filled-blue circles represent the real data of our final sample. We find that the both the real and simulated LF slopes randomly scatter above and below the input -2 slope. Thus stochastic scatter is not responsible for the trends found between LF slope and galaxy properties.

\subsubsection{Resolution effects}

Changes in the physical resolution of an image (i.e. due to galaxy distance) can change the degree to which distinct sources can be distinguished from one another. Consequently resolution can affect the slope of the LF by changing the number of sources in various luminosity bins. Greater galaxy distances (i.e. the FWHM is probing larger physical scales) can blend nearby sources into a single brighter source, thus flattening the LF slope by decreasing the number of faint sources and increasing the number of bright sources.

In a study of $\mathrm{H} \alpha$ sources (i.e. star-forming $\mathrm{H}$ II regions), Kennicutt et al. (1989) degraded the FWHM of $\mathrm{H} \alpha$ images of two nearby galaxies at evenly spaced intervals from 30 to $900 \mathrm{pc}$ scales, and found that the shape of the LF showed no significant change below 200 pc scales (for star clusters, see also: Randriamanakoto et al. 2013). At physical scales between 200 and 400 pc, the resolution experiments of Kennicutt et al. (1989) showed a loss of sources at lower luminosities, but no significant change in the bright end of the LF slope. Conversely, Scoville et al. (2001) found that the highresolution HST $\mathrm{H} \alpha \mathrm{LF}$ of M51 had a steeper LF slope by $0.2-0.5$ in $\alpha$ when compared to various ground-based LF slopes, where the physical scales probed by HST for M51 is $\sim 10$ pc and is $50-100$ pc for the different ground-based studies [see also Pleuss et al. (2000)].

Another factor to consider when comparing the LF slopes based on imaging which probe different physical scales is the fractal picture of star formation. Many studies on the clustering of young stars and star-forming regions have found similar luminosity distributions when probing a wide range of physical scales (i.e. from a few to 100 s of parsecs). Bastian et al. (2007) tested the change in the LF slope in M33 for different physical scales, and found no significant differences when probing $10-100 \mathrm{pc}$ scales. If star formation truly follows a fractal, or scale-free, distribution, then the LF slope should remain constant (at least when probing below a reasonably moderate physical scale). The results of Kennicutt et al. (1989) and Bastian et al. (2007) suggest that the LF slope should be not be significantly impacted by distance if probing a physical scale less than a few hundred parsecs. 


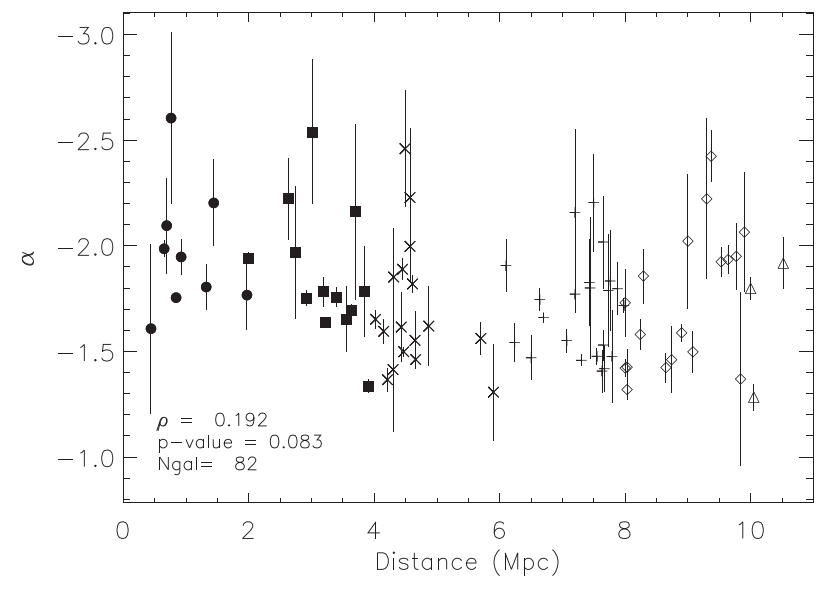

Figure 20. A plot of the LF slope $(\alpha)$ versus distance similar to that of Fig. 10. We find no trend between these two quantities indicating that resolution effects due to distance has little or no effect on our LF slope-galaxy property relationships.

The maximum distance of the 82 galaxies with clean LFs studied in this work is $\sim 10.5 \mathrm{Mpc}$, where the FWHM of the FUV image probes a physical scale of $\sim 250 \mathrm{pc}$. Thus we do not anticipate that the blending of sources due to distance will significantly impact our results. However, we perform several experiments to test the significance of distance/resolution may have on our LF slopes. As a first-order check, we examine the relationship between LF slope $(\alpha)$ and distance in Fig. 20. We find no correlation between $\alpha$ and distance, which is supported by the small Spearman correlation coefficient $(\rho=0.192)$. The lack of correlation between $\alpha$ and distance indicates that distance has a small or no effect on $\alpha$, but does not rule out the effect entirely since the magnitude of this effect may be small.

Another check on the effect of resolution is to examine the distribution of distances in the trends between $\alpha$ and the two properties that show the strongest correlations: SFR and $\Sigma_{\mathrm{SFR}}$. A visual inspection of both Figs 14 and 16 show that the filled symbols, which represent the closest galaxies, show a similar trend to that of the entire sample and span nearly the entire range of properties in both $\alpha$ and galaxy property. The persistence of these trends for the closest galaxies also suggests that resolution effects due to distance cannot be entirely responsible for the trends found between $\alpha$ and the galaxy properties of SFR and $\Sigma_{\text {SFR }}$.

Despite no clear trend between $\alpha$ and distance and the similar trend between $\alpha$ and star formation properties for the closest galaxies, it is possible that the strength of these trends may be exaggerated by resolution effects due to distance. To quantify this effect, we simulate the LF slopes of NGC0598 (M33; distance $0.8 \mathrm{Mpc}$ ) and NGC0300 (distance $\sim 2.0 \mathrm{Mpc}$ ) at increased distances by smoothing the GALEX FUV FWHM to larger pixel values, thus probing larger physical scales. The 5 arcsec native resolution of the FUV images corresponds to a physical scale of $24 \mathrm{pc}$ and $48 \mathrm{pc}$ for NGC0598 and NGC0300, respectively. In addition, the FUV native resolution at $10.5 \mathrm{Mpc}$ (the maximum distance of our sample) corresponds to a $250 \mathrm{pc}$ physical scale. We degrade the native resolution GALEX FUV image to equally spaced 30 pc physical scales out to $270 \mathrm{pc}$ to simulate the effects of distance on LF slope.

Our smoothing procedure takes into account the smoothing of FUV images to a larger FWHM values, the decrease of flux from sources due to an increased distance, and the increase of Poisson noise due to the decrease in flux for sources. For each physical scale we perform the following operations: (1) convert the native resolution image's pixel units into counts, (2) convolve the native resolution FUV image with the appropriate Gaussian to produce a smoothed image with a FWHM equal to the desired physical scale, (3) scale down the image counts due to distance, (4) rebin the smoothed image (i.e. increase the size of the pixels) to recover the 5 arcsec FWHM of the native FUV image, (5) draw a Poisson deviate per pixel to increase the Poisson noise, and (6) convert the resultant image back into the original pixel units. This procedure effectively yields GALEX FUV observations as if they were located at increased distances.

With the distance simulated images constructed, we then use identical methods to those used on the native resolution image to identify FUV regions, perform photometry, and generate LFs for each simulated image. We visually verify that all obvious sources are identified in our simulated images. In addition, since our original analysis required a dust correction for individual sources via the $L_{24} / L_{\mathrm{FUV}}$ luminosity ratio, we have also smoothed the Spitzer $24 \mu \mathrm{m}$ image to the corresponding physical scales using the same procedure to that of the FUV images.

Rebinning the smoothed images recovers the native resolution in pixels, thus, rebinning facilitates using the same photometry parameters (in pixels), the same aperture correction relationships derived in Section 4.3, and the same dust correction relationship derived in Section 4.4 to measure the total flux for each source in the smoothed images.

We then fit a power law to the LF of simulated sources using the same binning and fitting methods as those for the native resolution images. Fig. 21 and Fig. 22 show the resultant LFs for NGC0598 and NGC0300, respectively, where each panel represents the LF for different physical scales (i.e. physical scale increases from left-toright and from top-to-bottom). For each galaxy, there is a general trend of lower numbers of sources in the lower luminosity bins at larger physical scales (i.e. greater distances). It is likely that the reduced numbers of faint objects is largely due to a combination of the reduced counts for sources and the subsequent increased Poisson noise for increasing simulated distances. These two effects would act to hide faint sources in the noise of the simulated images.

It is reasonable to anticipate that fewer numbers of faint sources would result in flatter LF slopes. However we find that the slope of the brighter luminosity bins do not change significantly across all simulated distance. These results are consistent with the resolution experiments performed by Kennicutt et al. (1989), where they found no significant change in LF slope below physical scales of $200 \mathrm{pc}$ and found fewer faint sources at physical scales between 200 and $400 \mathrm{pc}$ but found no change on the bright end of the LF shape.

We also note that the reduced number of fainter sources indicate that the completeness (and detection) limits of our simulated images is increased for greater simulated distances. However, our fitting method takes into account varying completeness limits by fitting a power law to luminosity bins at and above the peak of the luminosity histogram, where incompleteness is likely to become prominent. Thus, our fitted LF slopes at greater simulated distances will continue to accurately describe the shape of the bright end of the LF. The increased completeness limits for our simulated images can be seen in both Figs 21 and 22 where the peak of the luminosity histogram (vertical-dashed lines) show a rough, overall trend towards higher luminosities at greater simulated distances. For instance the top three panels of Fig. 21 show a peak histogram luminosity of $36.7,37.0$, and $37.2 \mathrm{erg} \mathrm{s}^{-1}$ for physical scales of $30 \mathrm{pc}, 60 \mathrm{pc}$, and $90 \mathrm{pc}$, respectively. 


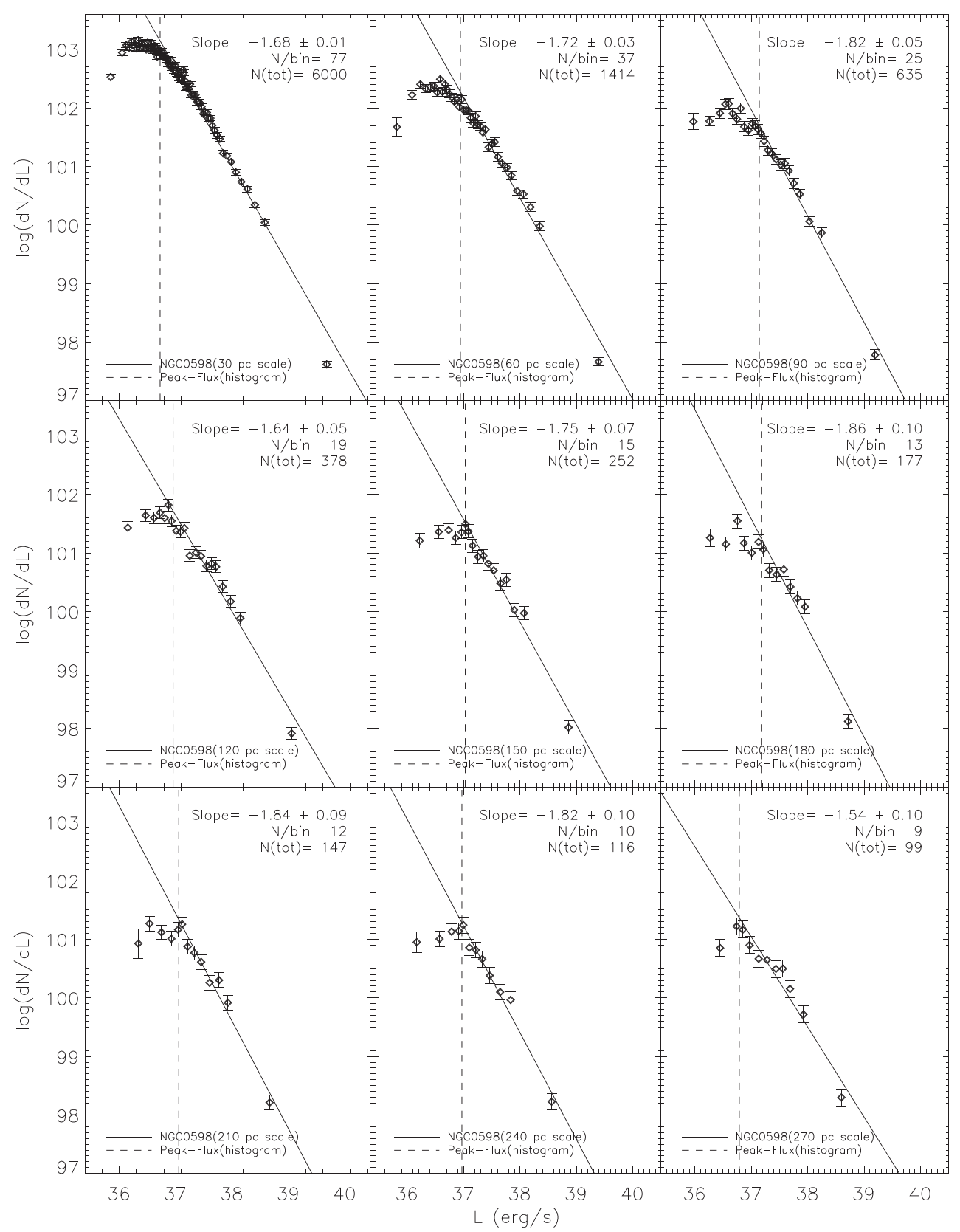

Figure 21. The simulated LFs for NGC0598 at increased distances, where each panel presents the results for increasing distances from left to right and from top to bottom (30-270 pc physical scales), and the dashed line represents the peak of the luminosity histogram for the sources found in each smoothed image. There is a general trend of decreasing numbers of sources in the faint luminosity bins for increasing distances. However, we find that each simulated LF slope are consistent with the native resolution LF slope within the errors.

The power-law fits to each of the simulated LFs are well behaved and yield similar slopes to the native resolution values within the fitted errors. This can be seen in a plot of the LF slope for each distance simulation versus the physical scale probed by each distance in Fig. 23, where the red diamonds and blue asterisks represent the simulated LF slope for NGC0598 and NGC0300, respectively. The horizontal dashed-red and dotted-blue lines represent the native resolution LF slopes for NGC0598 and NGC0300, respectively.

There is no clear trend between LF slope and the simulated distance for either galaxy, and we find that the simulated LF slopes are consistent with the native resolution LF slopes $(-1.75$ and -1.94 for NGC0598 and NGC0300, respectively) within the error bars of the simulated slopes. The median simulated slopes are -1.75 and -1.85 with a standard deviation of 0.11 and 0.12 for NGC0598 and NGC0300, respectively. The lack of a trend between simulated LF slope and distance suggests that the blending of sources due to distance does not significantly affect the trends found between LF slope and SFR properties.

We note that a lack of a change in the LF slope with increasing distance is only valid when measuring the bright end of the LF and so long as the lower limit of the fit is allowed to adjust to an appropriate luminosity given the completeness limit. Although we find no significant change in the bright end of the LF slope at greater 


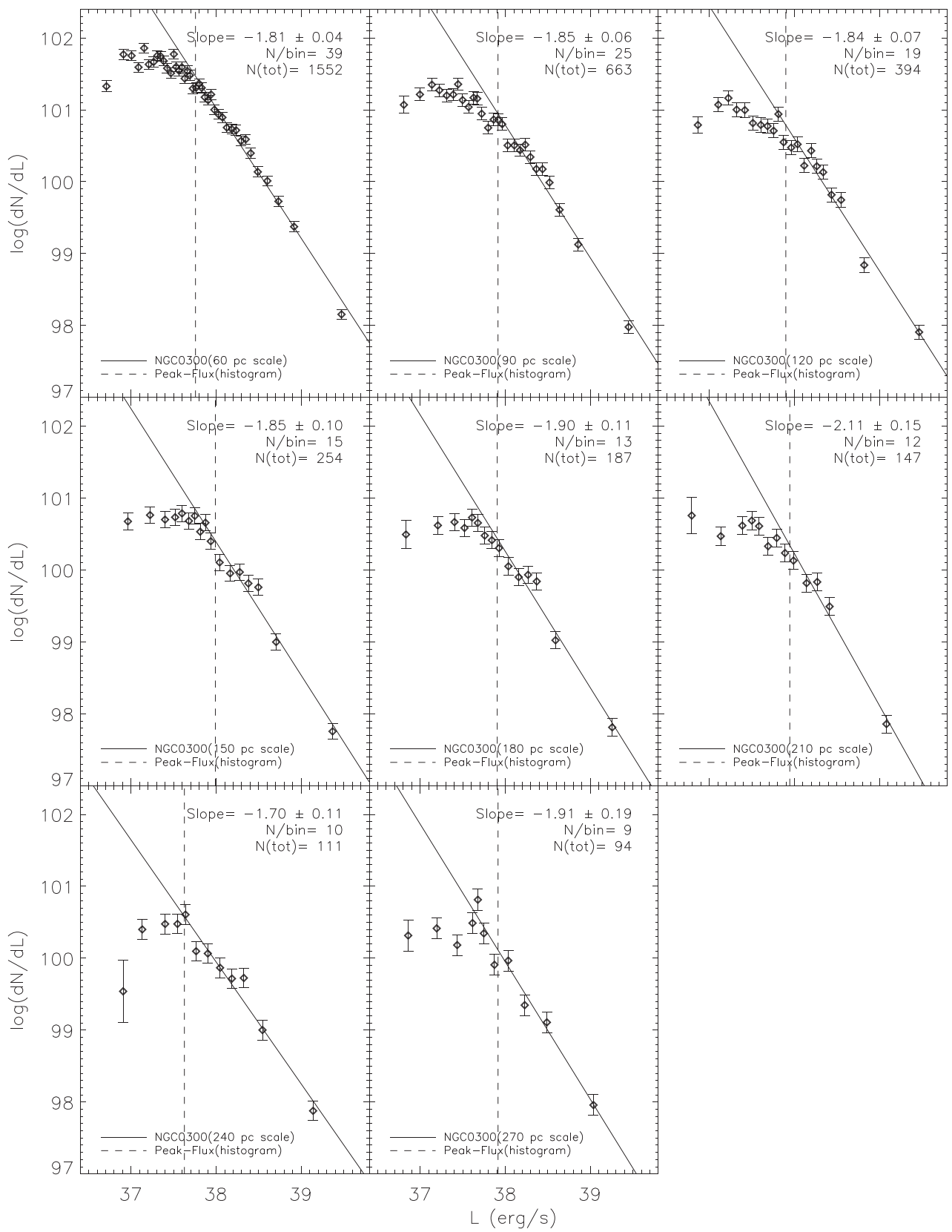

Figure 22. The simulated LFs for NGC0300 at increased distances, where each panel presents the results for increasing distances from left to right and from top to bottom (60-270 pc physical scales), and the dashed line represents the peak of the luminosity histogram for the sources found in each smoothed image. There is a general trend of decreasing numbers of sources in the faint luminosity bins for increasing distances. However, we find that each simulated LF slope are consistent with the native resolution LF slope within the errors.

distances, we do find that the faint end does appear to flatten due to the loss and/or blending of faint sources. However, since our LF slopes are derived for the bright end of the LF and our LF fits allow for a variable lower limit based on the completeness limit, we conclude that our trends between LF slope and galaxy properties are robust to blending due to distance.

A caveat of this analysis is the effect of blending due to the filling factor (or number density) of star-forming regions in galaxies with smaller diameters. When comparing the LF slopes of two galaxies at the same distance with the same number of regions but with different effective areas can conceivably blend sources, thus possibly flattening the LF slope of the galaxy with the smaller diameter (i.e. higher SFR per area). As a first-order attempt to quantify this effect we have examined any trends between LF slope and area $\left(\mathrm{kpc}^{2}\right)$ and the number of regions per area $\left(\# / \mathrm{kpc}^{2}\right)$. We find no trend between alpha and these two quantities $(\rho \sim 0.22$ and $\sim$ 0.11 for area and the number of regions per area, respectively); for brevity we do not show these plots. However, the plot of LF slope versus $N$ per area may not be a definitive test as the true number of sources cannot be known without HST resolution. We plan to simulate this effect in an upcoming paper looking at the LFs of star clusters with HST NUV imaging. 


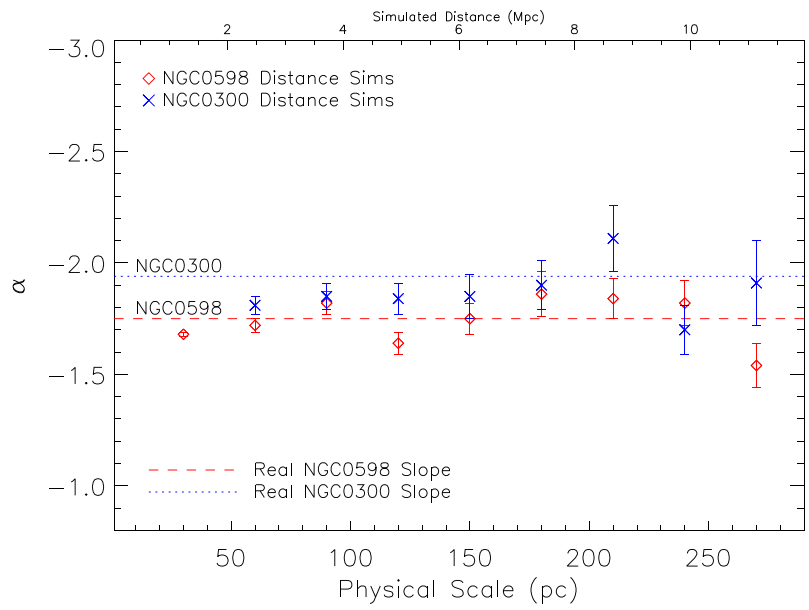

Figure 23. The combined LF slope results for our distance simulations where the red diamonds represent the simulated LF slopes for NGC0598, the blue-dotted line represents the LF slope measured on the native resolution image of NGC0598, the blue asterisks represent the simulated LF slopes for NGC0300, and the red-dashed line represents the LF slope measured on the native resolution image of NGC0598. We find no clear trend between the simulated LF slopes with distance. In addition, we find that the simulated LF slopes are consistent with the native resolution LF slope within the errors.

\subsection{Comparison to previous trends}

There are only a handful of studies that have previously examined trends between the LF slope and global galaxy properties. Of these previous studies, the majority have used $\mathrm{H}$ II regions (i.e. $\mathrm{H} \alpha$ identified sources; Kennicutt et al. 1989; Elmegreen \& Salzer 1999; Youngblood \& Hunter 1999; van Zee 2000; Thilker et al. 2002), one using Pa $\alpha$ identified $\mathrm{H}_{\text {II }}$ regions (Liu et al. 2013), and one using HST F814W ( $\sim$ I band) identified star clusters (Whitmore et al. 2014). These previous studies examined between 10 and 35 galaxies, where only the galaxy samples of Kennicutt et al. (1989); Elmegreen \& Salzer (1999); Whitmore et al. (2014) contained a range of morphology (i.e. early-type to irregular galaxies). Unfortunately, the other previous studies examined either only irregular galaxies (Youngblood \& Hunter 1999; van Zee 2000) or only later-type spirals (Thilker et al. 2002; Liu et al. 2013). For each of these studies, the authors purport that any trends between LF slope and galaxy property are inconclusive for their galaxy sample.

As a first step towards comparing our trends to those of previous trends, we provide a brief discussion on the effects of LFs derived from different wavelengths. Our star-forming regions are derived from GALEX FUV sources which probe different ages compared to previous studies. FUV wavelengths probe ages less than $100 \mathrm{Myr}$, while regions identified at $\mathrm{H} \alpha$ wavelengths probe ages less than $10 \mathrm{Myr}$ and star clusters identified at HST F814W wavelengths probe ages $\sim$ a few Gyr.

Previous studies have examined the effects of age on the shape of LFs and found that populations of older star-forming regions tend to have steeper LF slopes (Oey \& Clarke 1998). This steepening is attributed to the dimming of bright star-forming regions due stellar evolution, where the luminous star-forming regions contain relatively more massive stars which have short main-sequence lifetimes. We can test this hypothesis by comparing the average LF slopes between $\mathrm{H} \alpha$, FUV, and F814W LFs. The studies of Kennicutt et al. (1989) and Elmegreen \& Salzer (1999) report an average LF slope of $-2 \pm 0.5$ for $\mathrm{H} \alpha$ regions, Whitmore et al.
(2014) reports an average LF slope of $-2.37 \pm 0.18$ for $\mathrm{F} 814 \mathrm{~W}$ star clusters, and we report an average $\alpha$ of $-1.76 \pm 0.30$ for FUV regions. The average LF slope for the oldest population, F814W star clusters, is steeper than both the $\mathrm{H} \alpha$ and FUV averages. However, we find that our FUV (100 Myr) average $\alpha$ value is consistent with $\mathrm{H} \alpha$ (10 Myr) studies.

Another caveat to address is the effect of incomplete sampling of the stellar IMF in low-mass (similarly low-luminosity) star-forming regions. Hermanowicz, Kennicutt \& Eldridge (2013) found that the FUV luminosities of $\mathrm{H}$ II regions scales with the $\mathrm{H} \alpha$ luminosities, but that this scaling breaks down at low luminosities (Log $\sim 37$ $\mathrm{erg} \mathrm{s}^{-1}$ ) due to incomplete sampling of the stellar IMF. Since our regions are identified at FUV wavelengths they are not likely to be affected by IMF sampling issues, but this does not rule out the possibility that previous $\mathrm{H} \alpha$ studies are affected by incomplete sampling of the IMF. However, we note that none of our LFs are have fitted luminosity bins below $\log \sim 37 \mathrm{erg} \mathrm{s}^{-1}$, thus we believe our comparison to previous $\mathrm{H} \alpha$ studies is valid.

We also note that making LF slope comparisons from different studies for individual galaxies is not straight forward. There are multiple factors that can change the shape of the LF (i.e. wavelength, resolution, LF identification methods, binning methods, and LF fitting methods) which may have competing effects on the LF slope. However, we believe that any trends found between LF slope and galaxy properties are robust if the regions were uniformly identified and the LFs were generated with uniform methods for a sample of galaxies. Consequently, we provide only a qualitative comparison of our LF slope trends with galaxy properties to those of previous studies which have galaxy samples with a range in galaxy properties and whose LFs have been generated via uniform methods.

\subsubsection{Galaxy type}

Kennicutt et al. (1989) and Elmegreen \& Salzer (1999) found a correlation between the LF slope and galaxy type, where earlier type galaxies (Sa-Sab) exhibit steeper slopes than later-type galaxies (Sbc-Sd) and irregulars (Sm-Irr). Whitmore et al. (2014) found a similar correlation between $\alpha$ and galaxy type when ignoring the earliest type galaxies $(T<4)$, where later-type galaxies and irregulars showed flatter slopes.

Our trend of $\alpha$ versus galaxy type is shown in Fig. 10, where we find no clear trend between LF slope and galaxy type. We find increased scatter in $\alpha$ for later-type galaxies, especially in the irregular galaxies $(T \geq 9)$ where the LF slopes span the entire range of $\alpha$ values for whole galaxy sample.

The disagreement between the results presented in this study and those from previous studies may be due to underlying galaxy properties and the number of galaxies in the latest galaxy type bins $(T \geq 9)$. Our final sample is dominated by these later-type galaxies where 42 galaxies have $T \geq 9$, thus our sample reflects the properties of typical irregular galaxies in the local universe. However, the significance of trends in the previous studies heavily rely on the flatter slopes of only a few irregular type galaxies in each study: three galaxies (LMC, SMC, and NGC4449) in Kennicutt et al. (1989), one galaxy (the Antennae; NGC 4038/39) in Whitmore et al. (2014), and three galaxies (NGC1156, NGC1569, and NGC2366) in Elmegreen \& Salzer (1999).

The Antennae is a starbursting merger remnant whose properties do not reflect normal star-forming galaxies, thus may be an outlier in this parameter space. Furthermore, Whitmore et al. (2014) reported that the significance of the trend between $\alpha$ and galaxy type is 
reduced by a factor of 2 when removing the Antennae galaxy. In addition, Kennicutt et al. (1989) reported that the dispersion in $\alpha$ among spirals of the same galaxy type is similar to the magnitude of the trend between $\alpha$ and galaxy type, leaving three irregular galaxies to support the trend. Finally, Elmegreen \& Salzer (1999) studied only 11 galaxies in total which contain only 3 irregular galaxies where the range of $\alpha$ among each galaxy type spans more than half the total range of the entire sample [see fig. 8 of Elmegreen $\&$ Salzer (1999)]. The irregular galaxies in each of the previous studies have enhanced SFRs compared to typical local universe irregulars and may indicate the star formation properties of these galaxies are responsible for the flatter LF slopes.

\subsection{2 $\mathrm{M}_{B}$}

Whitmore et al. (2014) found a weak correlation between $\alpha$ and the absolute $B$-band magnitude $\left(M_{B}\right)$, where brighter galaxies tend to have steeper slopes. Fig. 11 shows a weak trend between $\alpha$ and $M_{B}$, where fainter galaxies tend to have steeper slopes; this is the opposite trend that was found by Whitmore et al. (2014). The discrepancy between these two trends is unclear. However, it is possible that the range covered in $M_{B}$ by Whitmore et al. (2014) is not large enough accurately characterize this relationship where the range in $M_{B}$ covered by Whitmore et al. (2014) is from -17.5 to $-21.5 \mathrm{mag}$ compared to our range of -10 to $-22 \mathrm{mag}$. It is also possible there exists no trend between $\alpha$ and $M_{B}$ since both our trend and Whitmore et al. (2014) trend are only weakly correlated.

\subsubsection{SFR}

Whitmore et al. (2014) found a correlation between $\alpha$ and global SFR, where lower SFR galaxies tend to have steeper slopes. We find a similar trend between these two quantities which can be seen in Fig. 14. We find that, in our analysis, the trend between $\alpha$ and SFR is weak-to-moderate, second only to $\Sigma_{\mathrm{SFR}}$. We note that our trend spans a larger range compared to Whitmore et al. (2014), where our data have a $\log (\mathrm{SFR})$ range of -5 to $1 \mathrm{M}_{\odot} \mathrm{yr}^{-1}$ and the data of Whitmore et al. (2014) span a range of -2 to $0.5 \mathrm{M}_{\odot} \mathrm{yr}^{-1}$.

Whitmore et al. (2014) attributes this trend to a 'size-of-sample effect' where higher SFRs create more star-forming regions, and in turn, are more likely to create brighter star-forming regions. This can affect the slope of the LF since low-SFR galaxies are less likely to form bright regions, thus creating a steeper slope.

The 'size-of-sample effect' has been examined by many studies of star clusters (Larsen 2002; Weidner, Kroupa \& Larsen 2004; Bastian 2008; Cook et al. 2012; Whitmore et al. 2014), and is statistical in nature. For example, randomly drawing star-forming regions from a power law with a slope of -2 from distributions with more regions yield a larger probability to draw a brighter region, and conversely you are less likely to draw a bright region from a distribution with small number statistics. This results in a correlation between the sample size (i.e. the total number of regions and subsequently SFR) and the luminosity of the brightest star-forming region $\left(L_{\max }\right)$. We find such a trend between SFR and $L_{\max }$ in Fig. 24 with a strong correlation $(\rho=0.904)$. We fit a line to the relationship which is described the equation:

$\log _{10}\left(L_{\max }\right)=1.1 \times \log _{10}(\mathrm{SFR})+41.9$.

The tight correlation in Fig. 24 suggests that the 'size-of-sample' effect is present in our sample, but our stochastic simulations of Section 8.1.1 show that low numbers of sources causes random scatter

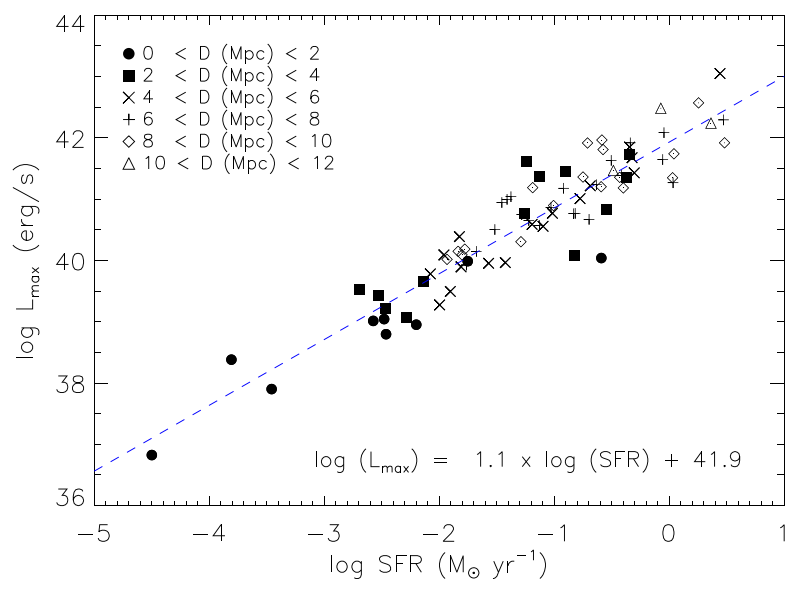

Figure 24. A plot of the luminosity of the brightest star-forming region $\left(L_{\max }\right)$ and SFR. We find a tight correlation with a correlation coefficient $(\rho)$ of 0.904 . Since the global SFR of a galaxy scales with the total number of sources, this tight trend indicates that a 'size-of-sample' effect is present in our sample. However, both the real and simulated LFs of galaxies with lownumber statistics show random scatter both above and below the canonical -2 LF slope suggesting that the 'size-of-sample' effect is not responsible for the trends between LF slope and galaxy properties.

both above and below the -2 slope, not systematically steeper slopes. Thus, we conclude that the steeper slopes in galaxies with low-number statistics are not due to a 'size-of-sample' effect.

It is interesting to note that previous star cluster studies have found a relationship between the LF slope $(\alpha)$, number of clusters detected (which is proportional to SFR; Whitmore et al. 2014), and the maximum mass (which scales with luminosity) of star clusters (e.g. Larsen 2002; Hunter et al. 2003; Bastian 2008). This relationship is derived in Hunter et al. (2003) and is described via:

$M_{\max } \propto N^{(1 / \alpha-1)}$.

Since $M_{\max }$ is proportional to $L_{\max }$ and the number of regions $(N)$ is proportional to SFR, we can use this relationship to estimate the combined LF slope of our star-forming regions by measuring the slope of the $L_{\max }$-SFR trend in Fig. 24. In log-log space, the exponent of equation (9) becomes the slope of Fig. 24 which we measure to be $\sim$ unity for our sample. This translates into a combined LF slope of -2 [it is actually +2 but Hunter et al. (2003) defined $\alpha$ such that the negative sign is already included in the equation].

The combined -2 LF slope matches the canonical LF slope, but is different than the median LF slope of our galaxy sample of -1.75 (average is -1.76). However, when we combine all star-forming regions into one composite sample and fit a LF, we find a bright-end slope of -1.93 ; very near the -2 value derived from the $L_{\max }-\mathrm{SFR}$ trend (see Section 8.4).

\subsection{Implications on the star formation process}

In the previous section we compared our LF slope trends to those of previous studies, where we found disagreement for galaxy type and $M_{B}$, however, found agreement for the trend between $\alpha$ and SFR. We also simulated other competing effects and found that the trends between $\alpha$ and star formation properties are not significantly affected. We conclude that the trends between $\alpha$ and star formation environment (SFR and $\Sigma_{\mathrm{SFR}}$ ) are real, and we discuss the implications of these trends on the star formation process in this section. 
The star formation process is ultimately governed by the gaseous starting material from which stars are made, and thus dependent on the host galaxy's interstellar medium properties: gas density $\left(\Sigma_{\mathrm{gas}} \equiv M_{\mathrm{gas}} / \mathrm{kpc}^{2}\right)$ and star formation efficiency $\left(M_{\star} / M_{\mathrm{gas}}\right)$. The Kennicutt-Schmidt law characterizes a tight relationship between $\Sigma_{\text {gas }}$ and $\Sigma_{\text {SFR }}$, where galaxies with higher $\Sigma_{\text {gas }}$ tend to have higher $\Sigma_{\text {SFR }}$ and the vertical normalization of this relationship represents the star formation efficiency (Kennicutt 1998). Furthermore, Kruijssen (2012) formulated a theoretical framework of star formation where higher $\Sigma_{\text {SFR }}$ and $\Sigma_{\text {gas }}$ environments can achieve higher star formation efficiencies, thus enhancing clustered star formation efficiency ( $\Gamma \equiv \mathrm{CFR} / \mathrm{SFR}$ ), which is supported by observational evidence (Goddard, Bastian \& Kennicutt 2010; Adamo, Östlin \& Zackrisson 2011; Cook et al. 2012).

$\Gamma$ represents the mass fraction of stars located in star clusters compared to those located in the field, thus $\Gamma$ probes the total mass (and similarly the luminosity) of star-forming regions in a galaxy. In this framework, as the $\Sigma_{\mathrm{SFR}}$ of a galaxy increases there is an increased fraction of stars in the densest, brightest parts of the star formation hierarchy (i.e. star-forming regions). We hypothesize that the flatter LF slopes seen in higher $\Sigma_{\text {SFR }}$ galaxies in our study are the result of higher $\Sigma_{\text {gas }}$ and higher star formation efficiencies creating relatively more bright star-forming regions, thus flattening the LF slope.

A simple test of this hypothesis is to examine any relationship between $\alpha$ and $\Gamma$. This is one of the goals for the second paper in this series where we will derive the masses of our star-forming regions and measure $\Gamma$ for our final galaxy sample.

\subsection{LF break}

Visual inspection of all LFs in Appendix A reveal no clear break in the LFs of any galaxy. However, 10 galaxies show reduced numbers in the brightest $\mathrm{L}$ bin compared to the extrapolated power-law fit (i.e. the data point of the brightest luminosity bin falls $>2 \sigma$ below the fit). A reduced number of sources in the brightest luminosity bin has been seen in both star cluster studies (e.g. Gieles et al. 2006; Bastian et al. 2012; Adamo et al. 2015) and the studies of giant molecular clouds (Rosolowsky et al. 2007) suggesting that the luminosity and mass functions of star-forming regions may have a high-luminosity and mass function turnover. In this section, we investigate if there exists a break in the LFs of star-forming regions.

Although we find no clear break in any individual galaxy's LF, we create a composite LF comprised of all sources from many galaxies to increase the number statistics of bright star-forming regions. The composite LF includes the star-forming regions of any galaxy with a measured inclination angle less than $60^{\circ}$ (plus those manually put back into the analysis; see Section 3). Consequently, the composite sample includes galaxies with poor LF fits and those with fewer than 30 total sources in addition to galaxies in our final sample.

Fig. 25 shows the composite LF of 134 galaxies where there is a clear break near a luminosity of $\sim 39.5 \mathrm{erg} \mathrm{s}^{-1}$. The bright end of the composite LF shows a slope of $\sim-1.93$; however, we find that the flatter slope at luminosities fainter than $\log (L) \sim 39.5 \mathrm{erg} \mathrm{s}^{-1}$ is due to incompleteness of more distant galaxies. Fig. 26 is a plot of the peak of the luminosity histogram (filled, black circles) and the $5 \sigma$ detection limit (open, blue diamonds) for each galaxy's FUV image versus the galaxy's distance. We find a relationship between these two luminosity limits and distance, where the limiting luminosity increases with distance and levels off near $\sim 39.5 \mathrm{erg} \mathrm{s}^{-1}$.

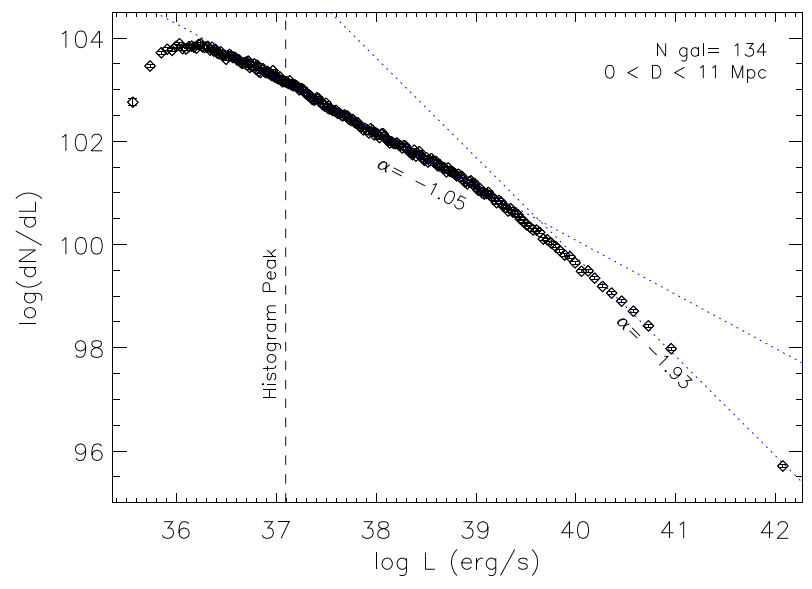

Figure 25. The composite LF where we have combined the star-forming regions of 134 galaxies to increase the number statistics of bright starforming regions. The $y$-axis is normalized to the same arbitrary number as those of the LFs of individual galaxies. We find a clear break at log $(L) \sim$ $39.5 \mathrm{erg} \mathrm{s}^{-1}$. However, we show that this break is due to different luminosity limits of more distant galaxies in the composite sample.

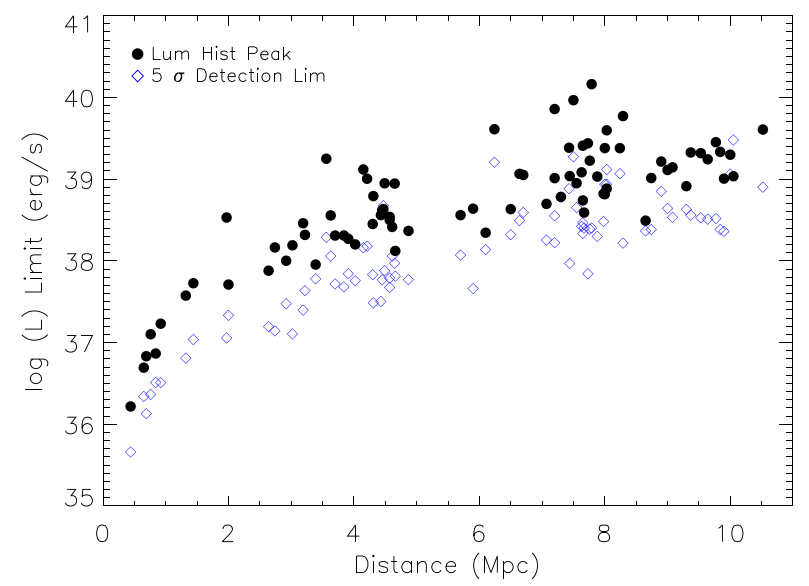

Figure 26. A plot of the peak of the luminosity histogram (filled, black circles) and the $5 \sigma$ detection limit (open, blue diamonds) for each galaxy's FUV image versus the galaxy's distance. We find a relationship between these two luminosity limits and distance, where the limiting luminosity increases with distance and levels off near $\sim 39.5 \mathrm{erg} \mathrm{s}^{-1}$. The break in Fig. 25 is due to reduced numbers of faint composite sources of galaxies at distance further away than $7 \mathrm{Mpc}$.

To understand how the break in the composite LF is related to the completeness limit of galaxies at different distances, we focus on the peak of the luminosity histogram in Fig. 26 since each galaxy's LF shows reduced numbers fainter than this luminosity due to incompleteness. The peak of the luminosity histogram levels off at $39.5 \mathrm{erg} \mathrm{s}^{-1}$, hence there will be little-to-no reduced numbers of composite sources brighter than $39.5 \mathrm{erg} \mathrm{s}^{-1}$ due to incompleteness. However, there will be reduced numbers of composite sources below $39.5 \mathrm{erg} \mathrm{s}^{-1}$ for all galaxies beyond a distance of $7 \mathrm{Mpc}$, where the peak in the luminosity histogram is, on average, near $\sim 39.5 \mathrm{erg} \mathrm{s}^{-1}$. In other words, the reduced numbers of sources in the composite LF fainter than $39.5 \mathrm{erg} \mathrm{s}^{-1}$ is caused by the incomplete luminosity bins of galaxies more distant than $7 \mathrm{Mpc}$. This suggests that the break seen in the composite LF of Fig. 25 likely does not reflect the true LF shape. We cannot confirm the existence of a break in the LF of star-forming regions with the data presented 
in this study. Given these results, we advocate for greater care of the completeness limits of galaxies in the samples of future composite LF studies.

\section{SUMMARY}

We have used the GALEX FUV images of 258 nearby galaxies to identify tens-of-thousands of star-forming regions and measured each galaxy's LF. Since our star-forming regions consist of point sources and extended sources with different morphologies, we measure the total flux of each source via an aperture correction based on CI, which is a measure of the extent of a source's radial profile. In addition, we use panchromatic data of star-forming regions in M51 from Calzetti et al. (2005) to derive a relationship between $24 \mu \mathrm{m} / \mathrm{FUV}$ luminosity ratio and dust extinction. We apply this dust correction to the FUV luminosities of our star-forming regions to account for the flux reprocessed by dust.

A comparison between two luminosity binning methods reveals that an average differences of -0.21 for LF slopes with equal luminosity-size bins and equal number bins. This difference is relatively small compared to the full range of luminosity slopes $(-2.8$ to -1.0 ), which indicates that our slopes are robust. In our final galaxy sample, we exclude any galaxy with an inclination angle less than $60^{\circ}, N<30$ total sources, and ill-behaved LFs leaving 82 galaxies in the final sample.

We find weak-to-moderate correlations between LF slope and several global galaxy properties; the strongest of which are SFR and SFR density $\left(\Sigma_{\text {SFR }}\right)$. We also find no correlation between LF slope and galaxy type. There is increased scatter for later-type galaxies $(T \geq 9)$ where we expect stochastic scatter to be present due to lownumber statistics in low-SFR, dwarf galaxies. In an examination of the effects of our choice of dust correction, aperture correction, the LF binning method choices on our trends between LF slope and galaxy properties we find the following: (1) our trends persist when using no dust correction and when assuming two different dust correction prescriptions; (2) we find that the trends persist when using two different aperture correction definitions and two luminosity binning methods; and (3) a stronger correlation between luminosity slope and $\left(\Sigma_{\mathrm{SFR}}\right)$ when using a weighted average of all four combinations of aperture correction and binning methods.

A careful examination of competing effects that can change the LF slope reveals that neither stochastic sampling of the LF in galaxies with low-number statistics nor the effects of blending due to distance can fully account for these relationships. We find that stochastic scatter due to low-number statistics results in random scatter to both flatter and steeper slopes in galaxies with fewer starforming regions. Since this scatter is not systematic, we conclude that our trends between LF slope and galaxy properties are not due to stochastic scatter. Examination of distance effects reveals no trend between the measured LF slopes and distance. Furthermore, we find that the closest galaxies $(D<2 \mathrm{Mpc})$ reproduce the trends between $\alpha$ and the galaxy properties of SFR and $\Sigma_{\mathrm{SFR}}$ for the entire sample. We also perform distance simulations on two nearby galaxies NGC0300 and NGC0598 (M33) located at a distance of $2.0 \mathrm{Mpc}$ and $0.8 \mathrm{Mpc}$, respectively. In these simulations we smooth and rebin the native resolution FUV images to increasing distances, re-extract sources, and re-measure the LF slopes. The resulting simulated slopes are consistent with the measured native resolution slopes within the errors and show no clear trend between simulated LF slope and the physical scales probed (FWHM range corresponding to $24-270 \mathrm{pc}$ ). These results suggest that the effects of resolution due to distance do not significantly affect the correlations seen between LF slope and global star formation properties.

We hypothesize that the trends found between LF slope and SFR properties may be connected to the star formation process via gas density and star formation efficiency since star formation is ultimately governed by the gaseous starting material. Support for this hypothesis can be found in previous studies where higher SFR densities correlate with (1) higher gas densities and (2) larger fractions of stars forming in star clusters $(\Gamma)$. This supporting observational evidence suggests that higher SFR density environments obtain higher star formation efficiencies, produce a larger fraction of stars in star clusters, produce relatively more bright star-forming regions, and thus flatten the LF slope. A direct test of this hypothesis is to measure $\Gamma$ for this galaxy sample and examine any relationship between LF slope and $\Gamma$. This is left for future work.

Finally, we test if a break in the LF accurately describes the LF shape by creating a composite LF of many galaxies to increase the number statistics of bright star-forming regions. We find a clear break in the composite LF near a luminosity of $\sim 39.5 \mathrm{erg} \mathrm{s}^{-1}$; however, we find that this break is not real. We attribute the break in the power law to a dearth faint sources where the most distant galaxies are affected by incompleteness below $L \sim 39.5 \mathrm{erg} \mathrm{s}^{-1}$.

\section{ACKNOWLEDGEMENTS}

We acknowledge the helpful comments provided by the referee. Support for this work, part of the Spitzer Space Telescope Legacy Science Program, was provided by National Aeronautics and Space Administration (NASA) through contract 1336000 issued by the Jet Propulsion Laboratory, California Institute of Technology under NASA contract 1407. This research has made use of the NASA/IPAC Extragalactic Database, which is operated by JPL/Caltech, under contract with NASA. IRAF is distributed by the National Optical Astronomy Observatory, which is operated by the Association of Universities for Research in Astronomy (AURA) under cooperative agreement with the National Science Foundation.

\section{REFERENCES}

Adamo A., Östlin G., Zackrisson E., 2011, MNRAS, 417, 1904

Adamo A., Kruijssen J. M. D., Bastian N., Silva-Villa E., Ryon J., 2015, MNRAS, 452, 246

Barnes K. L., van Zee L., Dale D. A., Staudaher S., Bullock J. S., Calzetti D., Chandar R., Dalcanton J. J., 2014, ApJ, 789, 126

Bastian N., 2008, MNRAS, 390, 759

Bastian N., Ercolano B., Gieles M., Rosolowsky E., Scheepmaker R. A., Gutermuth R., Efremov Y., 2007, MNRAS, 379, 1302

Bastian N. et al., 2012, MNRAS, 419, 2606

Beckman J. E., Rozas M., Zurita A., Watson R. A., Knapen J. H., 2000, AJ, 119,2728

Berg D. A. et al., 2012, ApJ, 754, 98

Bertin E., Arnouts S., 1996, A\&AS, 117, 393

Bik A., Lamers H. J. G. L. M., Bastian N., Panagia N., Romaniello M., 2003, A\&A, 397, 473

Boquien M. et al., 2015, A\&A, 578, A8

Bradley T. R., Knapen J. H., Beckman J. E., Folkes S. L., 2006, A\&A, 459, L13

Buat V. et al., 2005, ApJ, 619, L51

Calzetti D., 2001, PASP, 113, 1449

Calzetti D., Armus L., Bohlin R. C., Kinney A. L., Koornneef J., StorchiBergmann T., 2000, ApJ, 533, 682

Calzetti D. et al., 2005, ApJ, 633, 871

Calzetti D. et al., 2007, ApJ, 666, 870

Cardelli J. A., Clayton G. C., Mathis J. S., 1989, ApJ, 345, 245 
Chandar R. et al., 2010, ApJ, 719, 966

Chandar R., Fall S. M., Whitmore B. C., 2015, ApJ, 810, 1

Cook D. O. et al., 2012, ApJ, 751, 100

Cook D. O. et al., 2014a, MNRAS, 445, 881

Cook D. O. et al., 2014b, MNRAS, 445, 899

Dale D. A. et al., 2009, ApJ, 703, 517

de Grijs R., Anders P., Bastian N., Lynds R., Lamers H. J. G. L. M., O’Neil E. J., 2003, MNRAS, 343, 1285

de Vaucouleurs G., de Vaucouleurs A., Corwin H. G., Jr, Buta R. J., Paturel G., Fouqué P., 1991, Third Reference Catalogue of Bright Galaxies. Volume I: Explanations and references. Volume II: Data for galaxies between $0^{h}$ and $12^{h}$. Volume III: Data for galaxies between $12^{h}$ and $24^{h}$. Springer-Verlag, New York

Draine B. T., 2003, ARA\&A, 41, 241

Ellison S. L., Patton D. R., Simard L., McConnachie A. W., 2008, ApJ, 672, L107

Elmegreen B. G., 2006, ApJ, 648, 572

Elmegreen B. G., 2010, in de Grijs R., Lépine J. R. D., eds, Proc. IAU Symp. 266, The nature and nurture of star clusters. Cambridge Univ. Press, Cambridge, p. 3

Elmegreen D. M., Salzer J. J., 1999, AJ, 117, 764

Eskew M., Zaritsky D., Meidt S., 2012, AJ, 143, 139

Gieles M., Larsen S. S., Bastian N., Stein I. T., 2006, A\&A, 450, 129

Goddard Q. E., Bastian N., Kennicutt R. C., 2010, MNRAS, 405, 857

Gouliermis D. A., Schmeja S., Klessen R. S., de Blok W. J. G., Walter F., 2010, ApJ, 725, 1717

Gouliermis D. A. et al., 2015, MNRAS, 452, 3508

Hao C.-N., Kennicutt R. C., Johnson B. D., Calzetti D., Dale D. A., Moustakas J., 2011, ApJ, 741, 124

Hermanowicz M. T., Kennicutt R. C., Eldridge J. J., 2013, MNRAS, 432, 3097

Hunter D. A., Elmegreen B. G., Dupuy T. J., Mortonson M., 2003, AJ, 126, 1836

Kennicutt R. C., Jr, 1998, ARA\&A, 36, 189

Kennicutt R. C., Jr, Edgar B. K., Hodge P. W., 1989, ApJ, 337, 761

Kennicutt R. C., Jr, Lee J. C., Funes, José G. S. J., Sakai S., Akiyama S., 2008, ApJS, 178, 247

Kroupa P., 2001, MNRAS, 322, 231

Kruijssen J. M. D., 2012, MNRAS, 426, 3008

Lada C. J., Lada E. A., 2003, ARA\&A, 41, 57

Larsen S. S., 2002, AJ, 124, 1393

Lee H., Grebel E. K., Hodge P. W., 2003, A\&A, 401, 141

Lee H., Zucker D. B., Grebel E. K., 2007, MNRAS, 376, 820

Lee J. C. et al., 2009, ApJ, 706, 599

Lee J. C. et al., 2011, ApJS, 192, 6

Leroy A. K., Walter F., Brinks E., Bigiel F., de Blok W. J. G., Madore B., Thornley M. D., 2008, AJ, 136, 2782

Liu G., Koda J., Calzetti D., Fukuhara M., Momose R., 2011, ApJ, 735, 63

Liu G., Calzetti D., Kennicutt R. C., Jr, Schinnerer E., Sofue Y., Komugi S., Egusa F., Scoville N. Z., 2013, ApJ, 772, 27

Maíz Apellániz J., Úbeda L., 2005, ApJ, 629, 873

Marble A. R. et al., 2010, ApJ, 715, 506
Martin D. C. et al., 2005, ApJ, 619, L1

McCrady N., Graham J. R., 2007, ApJ, 663, 844

McGaugh S. S., 1991, ApJ, 380, 140

McGaugh S. S., Schombert J. M., 2014, AJ, 148, 77

McGaugh S. S., Schombert J. M., 2015, ApJ, 802, 18

Meidt S. E. et al., 2014, ApJ, 788, 144

Momcheva I. G., Lee J. C., Ly C., Salim S., Dale D. A., Ouchi M., Finn R., Ono Y., 2013, AJ, 145, 47

Morrissey P. et al., 2007, ApJS, 173, 682

Moustakas J., Kennicutt R. C., Jr, Tremonti C. A., Dale D. A., Smith J.-D. T., Calzetti D., 2010, ApJS, 190, 233

Murphy E. J. et al., 2011, ApJ, 737, 67

Oey M. S., Clarke C. J., 1998, AJ, 115, 1543

Oh S.-H., de Blok W. J. G., Walter F., Brinks E., Kennicutt R. C., Jr, 2008, AJ, 136, 2761

Osterbrock D. E., Ferland G. J., 2006, in Book Review: Astrophysics of Gaseous Nebulae and Active Galactic Nuclei, 2nd edn. University Science Books, 2005. South Orange, New Jersey

Pleuss P. O., Heller C. H., Fricke K. J., 2000, A\&A, 361, 913

Prescott M. K. M. et al., 2007, ApJ, 668, 182

Randriamanakoto Z., Vaisanen P., Ryder S., Kankare E., Kotilainen J. K., Mattila S., 2013, MNRAS, 431, 554

Rosolowsky E., Keto E., Matsushita S., Willner S. P., 2007, ApJ, 661, 830

Salim S., Lee J. C., Ly C., Brinchmann J., Davé R., Dickinson M., Salzer J. J., Charlot S., 2014, ApJ, 797, 126

Scoville N. Z., Polletta M., Ewald S., Stolovy S. R., Thompson R., Rieke M., 2001, AJ, 122, 3017

Thilker D. A., Walterbos R. A. M., Braun R., Hoopes C. G., 2002, AJ, 124, 3118

Thilker D. A. et al., 2007, ApJS, 173, 538

Tully R. B., Fisher J. R., 1977, A\&A, 54, 661

van Zee L., 2000, AJ, 119, 2757

Weidner C., Kroupa P., Larsen S. S., 2004, MNRAS, 350, 1503

Whitmore B. C. et al., 2014, ApJ, 795, 156

Youngblood A. J., Hunter D. A., 1999, ApJ, 519, 55

Zhang Q., Fall S. M., 1999, ApJ, 527, L81

\section{SUPPORTING INFORMATION}

Additional Supporting Information may be found in the online version of this article:

Table 1. General galaxy properties.

(http://www.mnras.oxfordjournals.org/lookup/suppl/doi:10.1093/ mnras/stw1694/-/DC1).

Please note: Oxford University Press is not responsible for the content or functionality of any supporting materials supplied by the authors. Any queries (other than missing material) should be directed to the corresponding author for the article. 


\section{APPENDIX A: ALL LUMINOSITY FUNCTION}

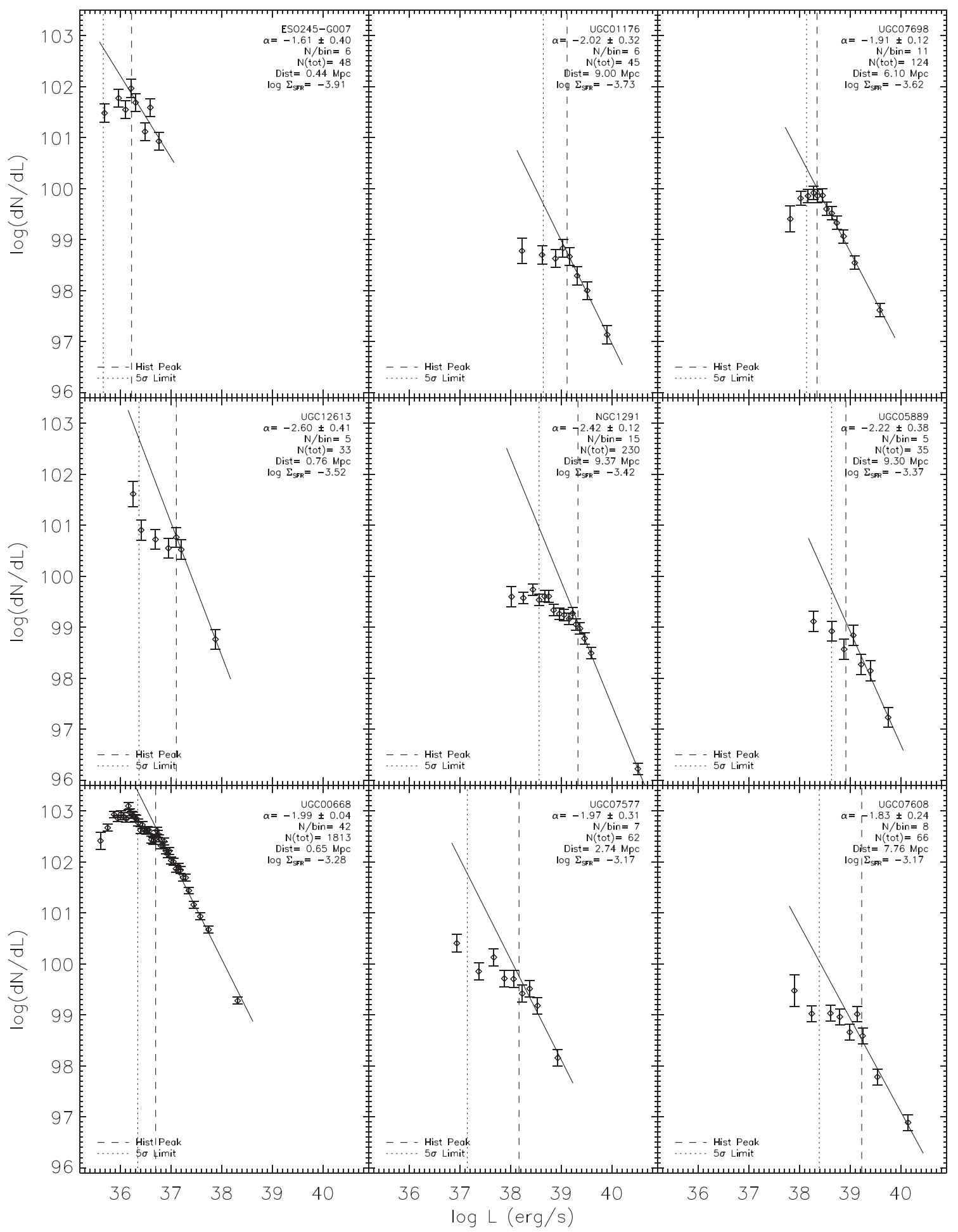

Figure A1. Figures A1-A10 show the LFs for all 82 galaxies in our final sample, where the panels have been sorted by $\Sigma_{\mathrm{SFR}}$. The $y$-axes for all LFs in all figures have been normalized to the same arbitrary number to facilitate comparisons of all luminosity functions. 


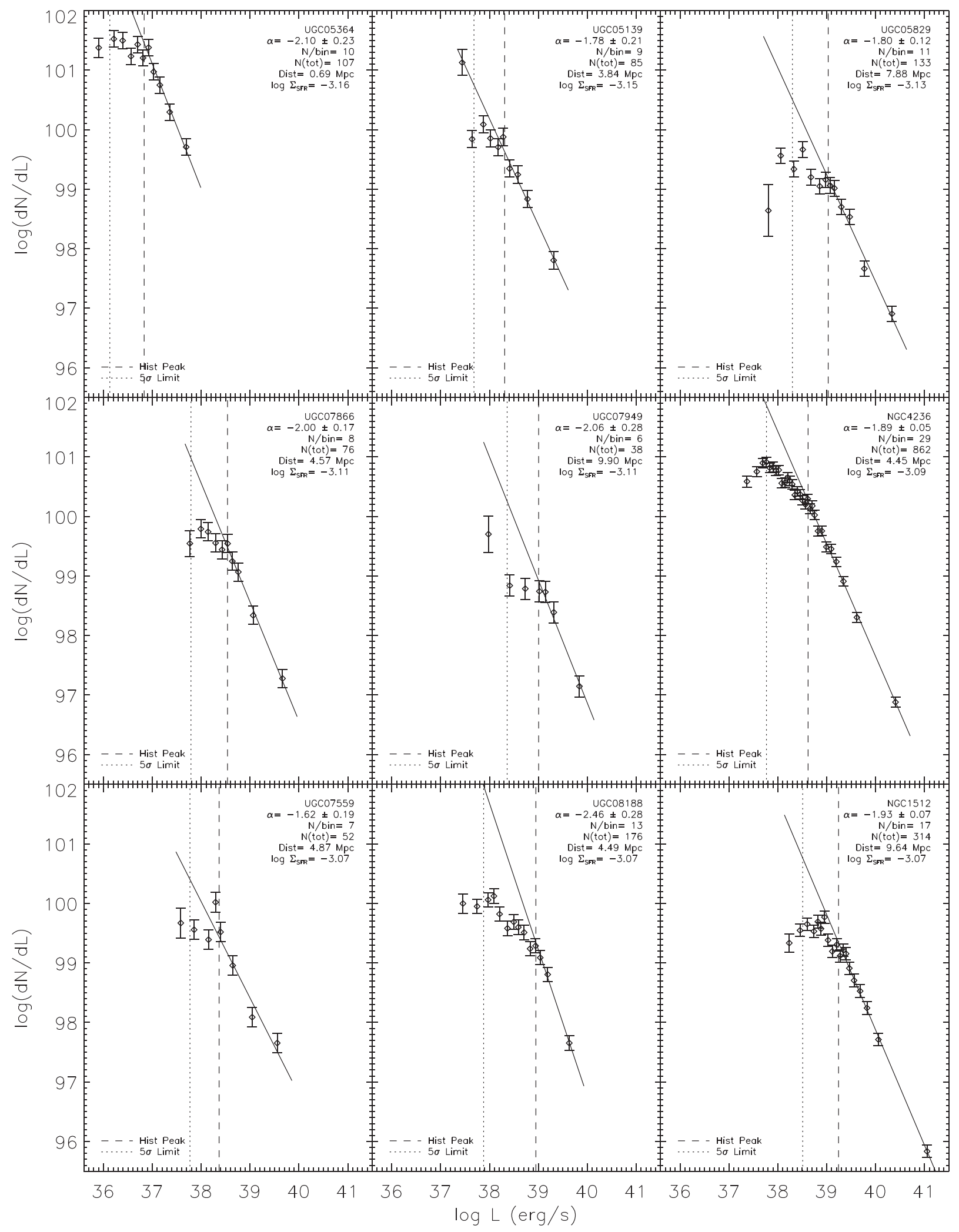

Figure A2 


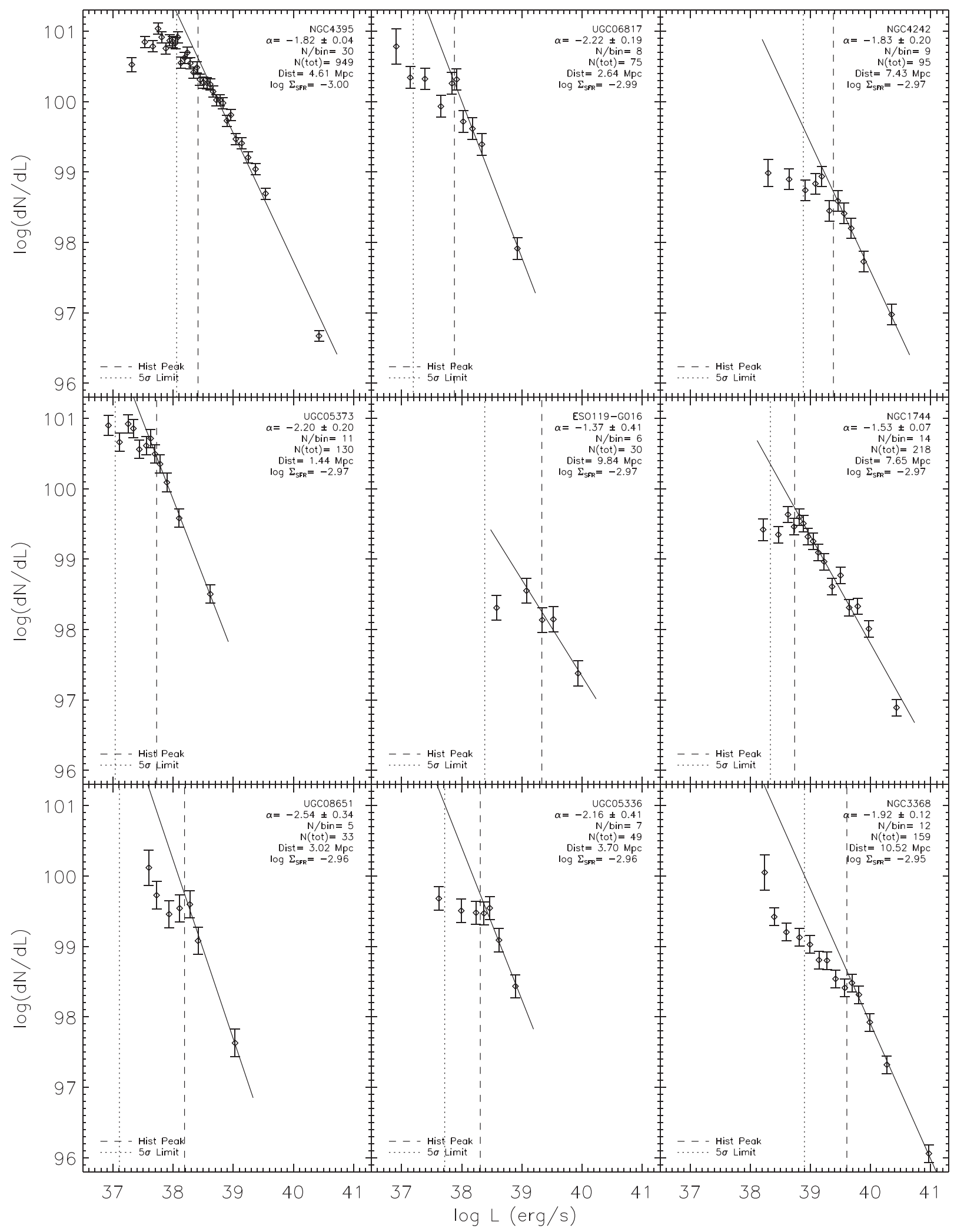

Figure A3 


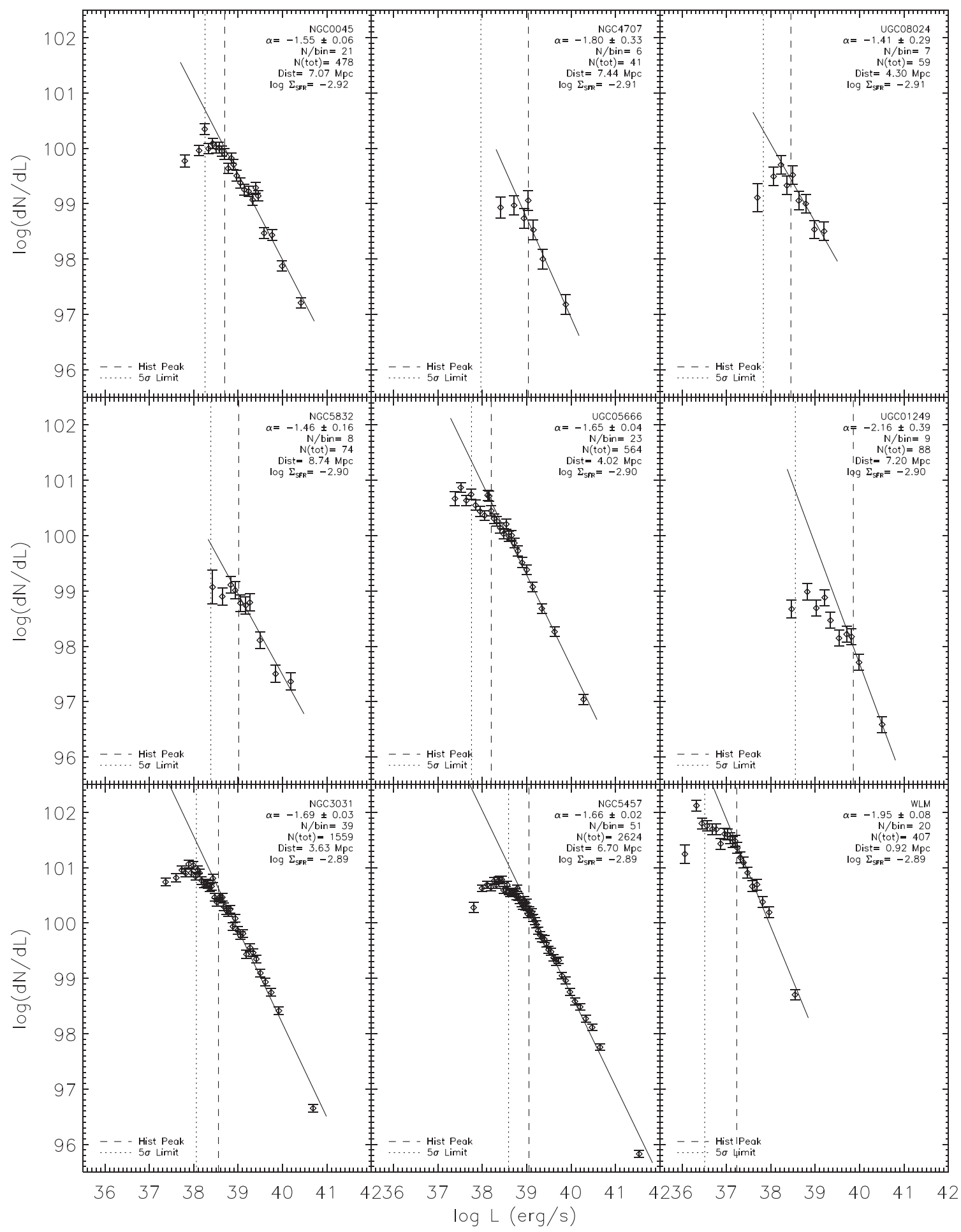

Figure A4 
D. O. Cook et al.

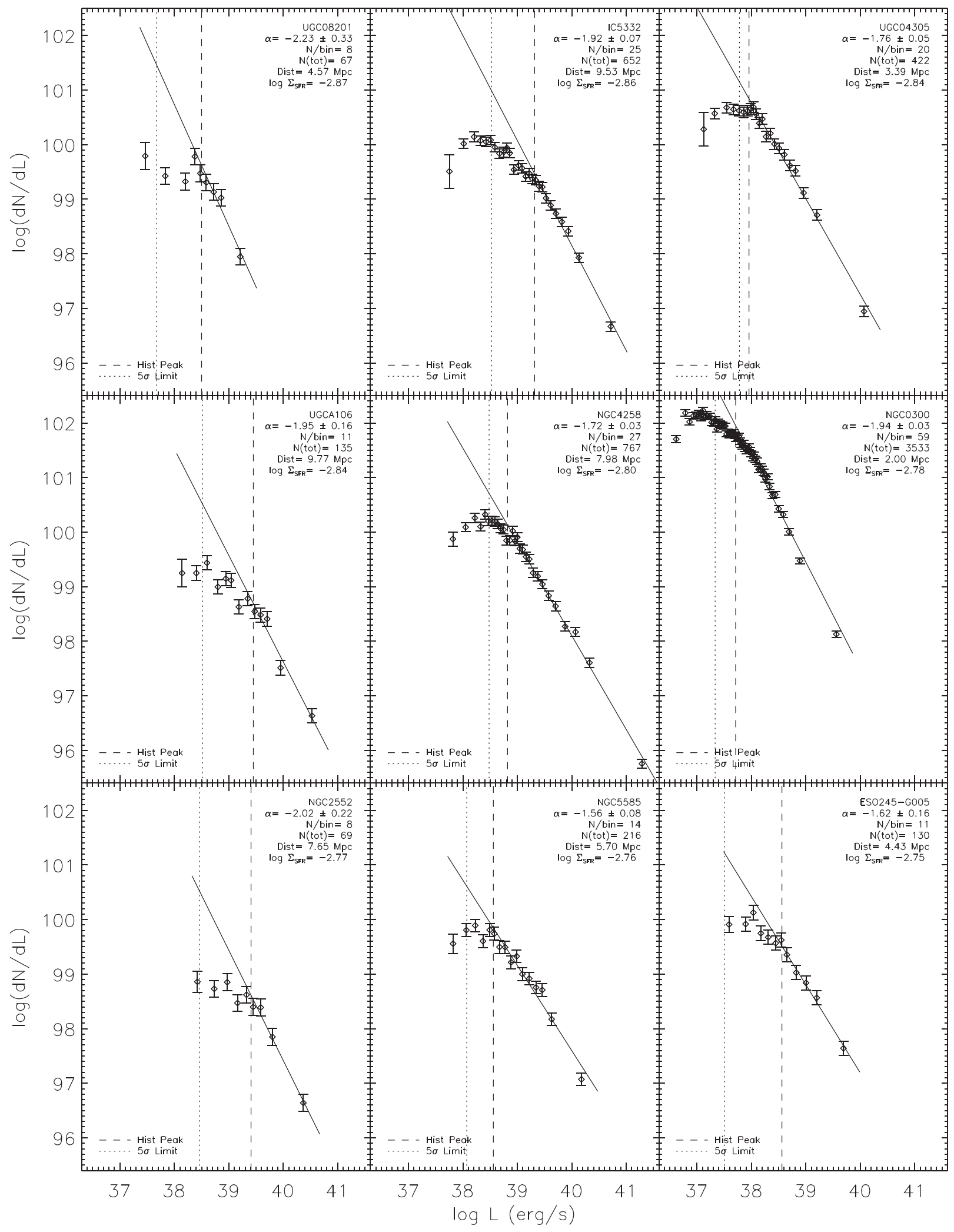

Figure A5 


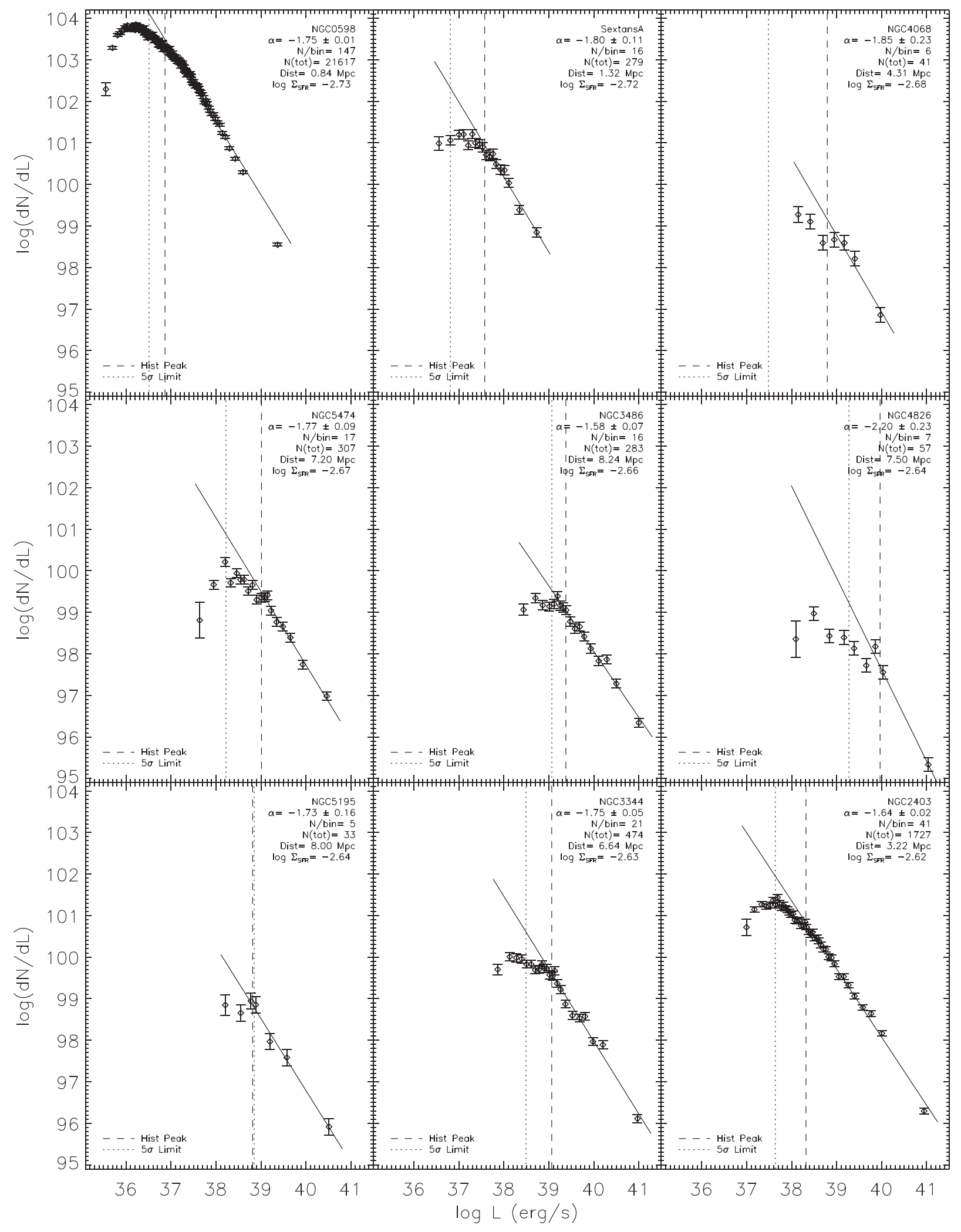

Figure A6 
D. O. Cook et al.

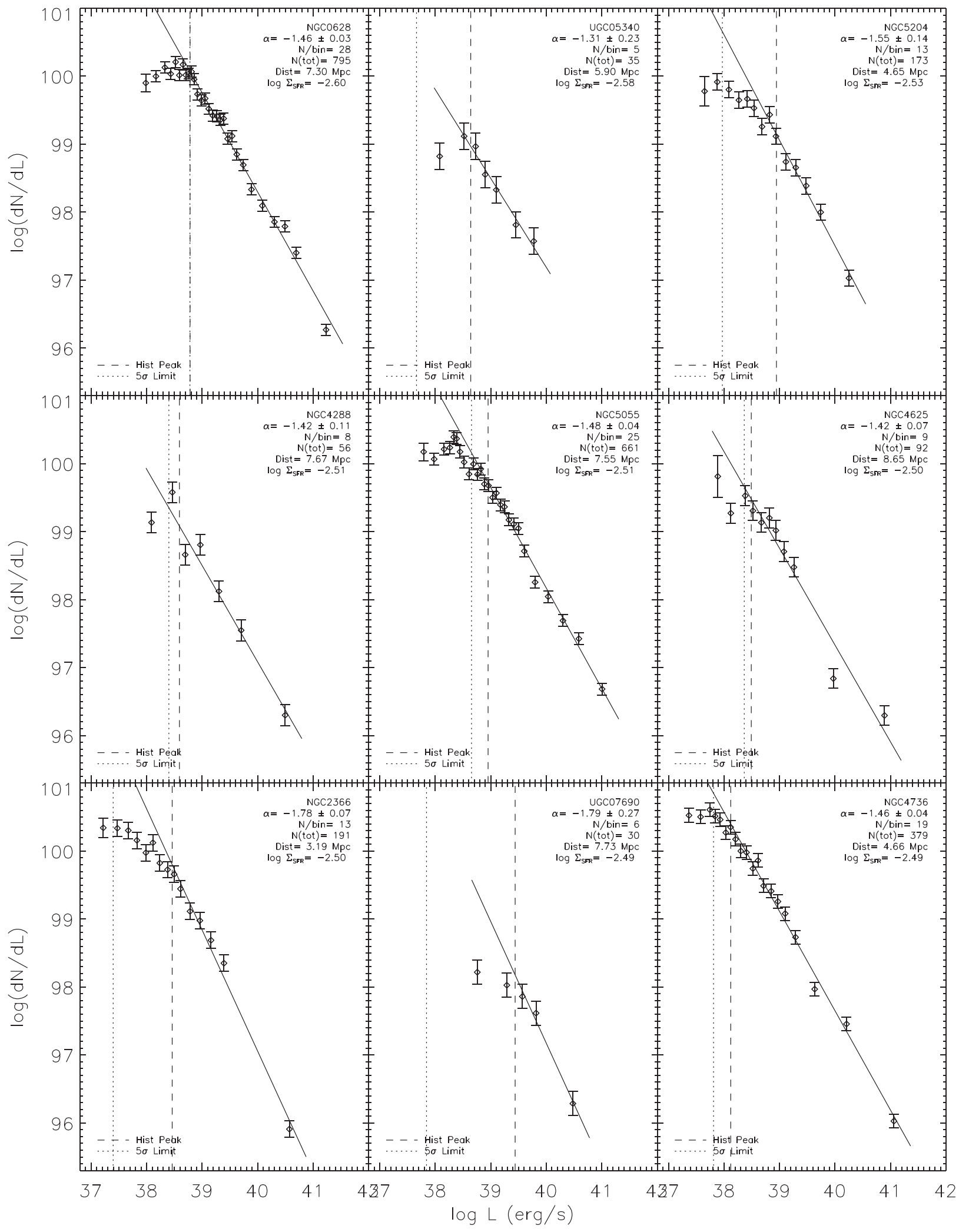

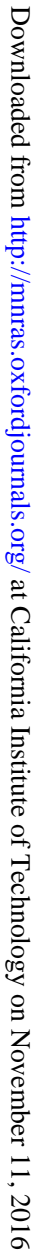

Figure A7 


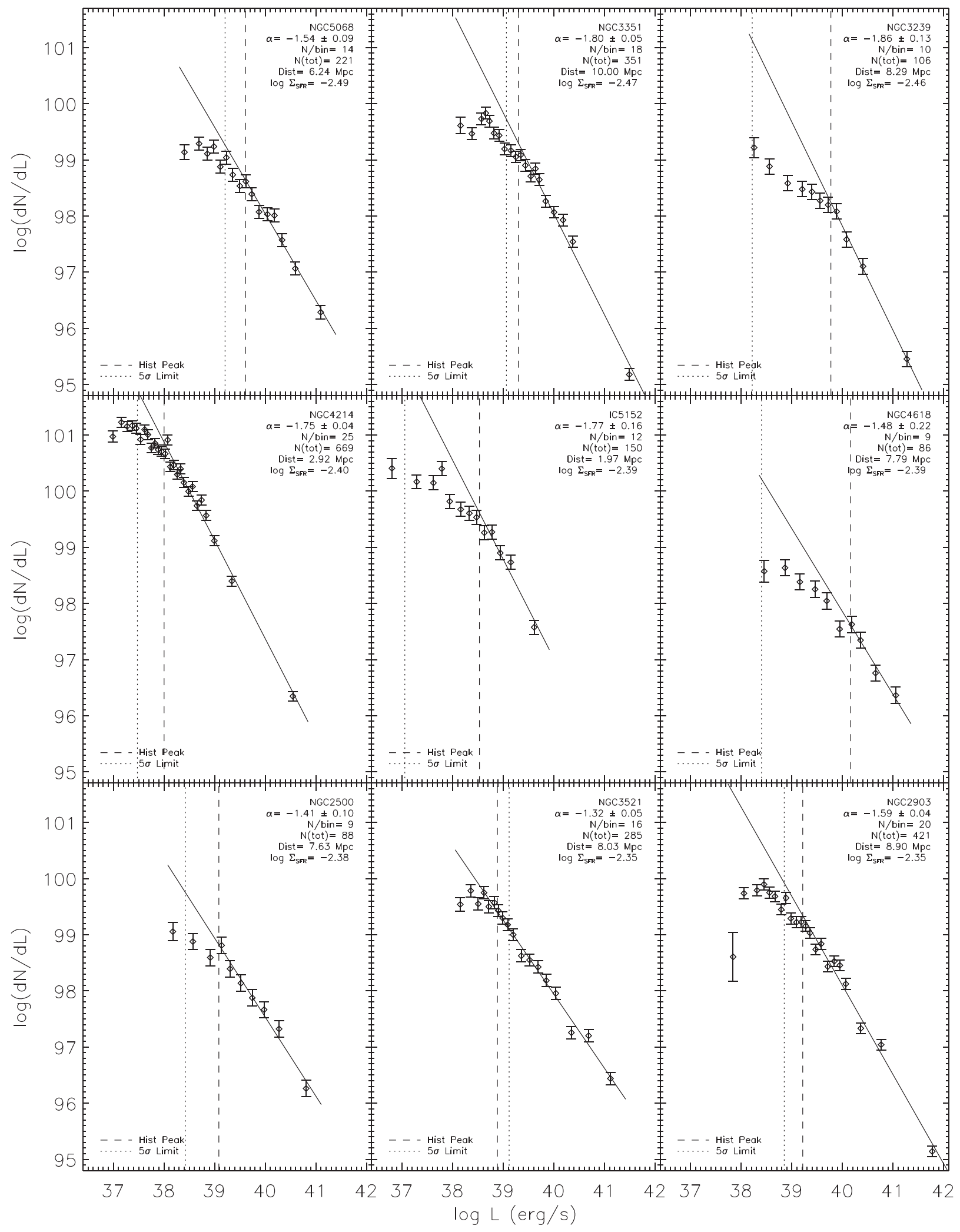

Figure A8 
D. O. Cook et al.

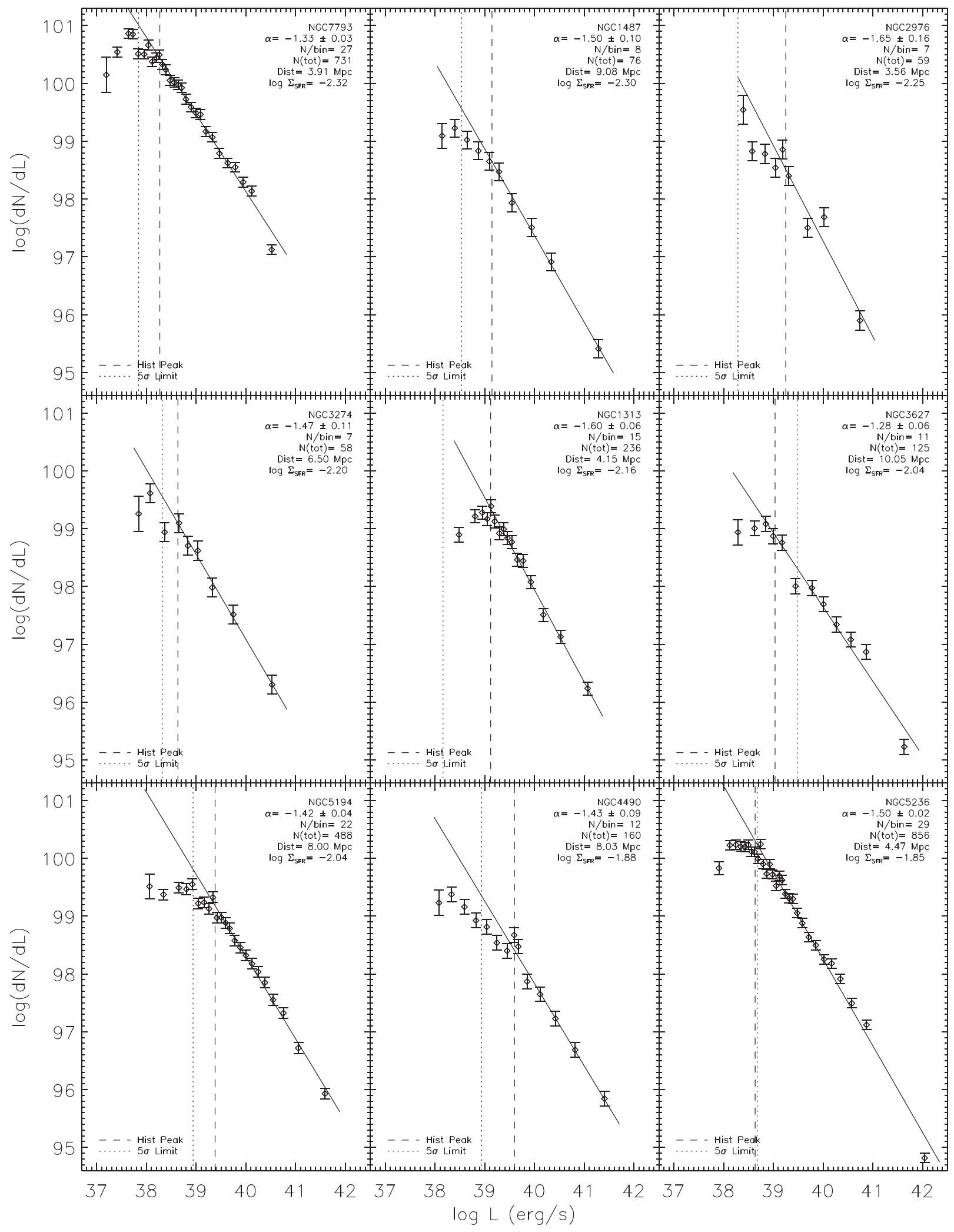

Figure A9 


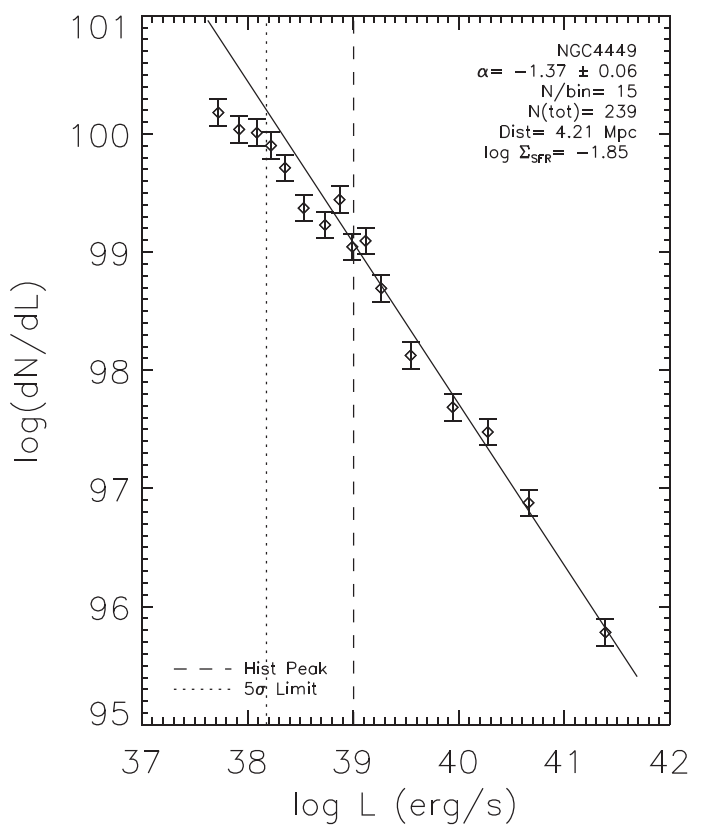

Figure A10

This paper has been typeset from a $\mathrm{T}_{\mathrm{E}} \mathrm{X} / \mathrm{L} \mathrm{T}_{\mathrm{E}} \mathrm{X}$ file prepared by the author. 